\title{
SYMMETRIC POLYNOMIALS AND DIVIDED DIFFERENCES IN FORMULAS OF INTERSECTION THEORY
}

\author{
PIOTR PRAGACZ $\left({ }^{1}\right)$ \\ Max-Planck-Institut für Mathematik \\ Gottfried-Claren Strasse 26, D-53225 Bonn, Germany \\ E-mail: pragacz@mpim-bonn.mpg.de
}

To the memory of Constantin Banica (1942-1991) and Krzysztof Jaczewski (1955-1994)

Introduction. The goal of this paper is at least two-fold. First we attempt to give a survey of some recent (and developed up to the time of the Banach Center workshop Parameter Spaces, February '94 $\left({ }^{2}\right)$ ) applications of the theory of symmetric polynomials and divided differences to intersection theory. Secondly, taking this opportunity, we complement the story by either presenting some new proofs of older results (and this takes place usually in the Appendices to the present paper) or providing some new results which arose as by-products of the author's work in this domain during last years.

Being in the past a good part of the classical algebraic knowledge (related for instance to the theory of algebraic equations and elimination theory), the theory of symmetric functions is rediscovered and developed nowadays (see, for example, the monograph [M1] of I. G. Macdonald or the booklet [L-S1] of A. Lascoux and M.-P. Schützenberger). Here, we discuss only some geometric applications of symmetric polynomials which are related to the present interest of the author. In particular, the theory of polynomials universally supported on degeneracy loci ([P3]) is surveyed in Section 1.

Divided differences appeared already in the interpolation formula of I. Newton [N, Liber III, p. 582, Lemma V: "Invenire lineam curvam generis parabolici, qua per data

1991 Mathematics Subject Classification: Primary 05E05, 14C15, 14C25, 14M15, 14N10, 57R20; Secondary 05E15, 14M12, 14H40, 14J60, 32S20, 55R40.

The paper is in final form and no version of it will be published elsewhere.

$\left({ }^{1}\right)$ During the preparation of this paper, the author greatly benefited from the hospitality of the Max-Planck-Institut für Mathematik in Bonn and was partially supported by the Alexander von Humboldt Stiftung and KBN grant No. 2 P301002 05.

$\left({ }^{2}\right)$ Several notes have been added on the galley proof; they provide information about some related results learned by the author after the above date. 
quotcunque puncta tran fibit."]. Their appearance in intersection theory is about twenty years old starting with the papers $[\mathrm{B}-\mathrm{G}-\mathrm{G}]$ of I. N. Bernstein, I. M. Gelfand and S. I. Gelfand and [D1,2] of M. Demazure. A recent work [F2] of W. Fulton has illuminated the importance of divided differences to flag degeneracy loci. This was possible thanks to the algebraic theory of Schubert polynomials developed recently by A. Lascoux and M.-P. Schützenberger ([L-S 2-6]).

The geometrical objects we study are: (ample) vector bundles, degeneracy loci of vector bundle homomorphisms, flag varieties, Grassmannians including isotropic Grassmannians, i.e. the parameter spaces for isotropic subspaces of a given vector space endowed with an antisymmetric or symmetric form, Schubert varieties and the parameter spaces of complete quadrics.

The algebro-combinatorial tools we use are: Schur polynomials including supersymmetric and $Q$-polynomials, binomial determinants and Pfaffians, divided differences, Schubert polynomials of Lascoux and Schützenberger, reduced decompositions in the Weyl groups and Young-Ferrers' diagrams.

The content of the article is as follows:

1. Polynomials universally supported on degeneracy loci,

2. Some explicit formulas for Chern and Segre classes of tensor bundles with applications to enumerative geometry,

3. Flag degeneracy loci and divided differences,

4. Gysin maps and divided differences,

5. Fundamental classes, diagonals and Gysin maps,

6 . Intersection rings of spaces $G / P$, divided differences and formulas for isotropic degeneracy loci - an introduction to [P-R 2-5],

7. Numerically positive polynomials for ample vector bundles with applications to Schur polynomials of Schur bundles and a vanishing theorem.

Apart of surveyed results, the paper contains also some new ones. Perhaps the most valuable contribution, contained in Section 5, is provided by a method of computing the fundamental class of a subscheme using the class of the diagonal of the ambient scheme. The class of the diagonal can be determined with the help of Gysin maps (see Section 5). This method has been applied successfully in [P-R5] and seems to be useful also in other settings. Other results that appear to be new are contained in Proposition 1.3(ii), Proposition 2.1 and Corollary 7.2. Moreover, the paper is accompanied by a series of appendices which contain an original material but of more technical nature than the main text of the paper. Some proofs in the Appendices use an operator approach and the operators involved are mostly divided differences. This point of view leads to more natural proofs of many results than the ones known before, and we hope to develop it in [L-L-P-T].

The following is the list of appendices:

A.1. Proof of Proposition 1.3(ii).

A.2. Proof of Proposition 2.1.

A.3. Recursive linear relations for $((J))$ and $[J]$.

A.4. A Gysin map proof of the formula from Example 3.5.

A.5. An operator proof of the Jacobi-Trudi identity. 
A.6. A Schur complex proof of the Giambelli-Thom-Porteous formula.

A. $\tau$. Corrigenda and addenda to some former author's papers.

Several open problems are stated throughout the text.

We use the opportunity to complete or correct some surveyed results. Moreover, we give, in Appendix A. $\tau$, an errata to some former author's papers.

Acknowledgement. This paper, being a revised and substantially extended version of the Max-Planck-Institut für Mathematik Preprint No. 92-16 Geometric applications of symmetric polynomials; some recent developments, is also an expanded version of two talks given by the author during the workshop Parameter Spaces; enumerative geometry, algebra and combinatorics. In fact, these talks were given in a linkage with two lectures by Alain Lascoux to whom the present paper owes a lot. At first, it was Lascoux who introduced me several years ago to this branch of mathematics and I wish to express to him my sincere gratitude. Secondly, I learned some material exposed in the present paper directly from Lascoux. These are: the divided-differences interpretation of the symmetrizing operators in Propositions 4.1-4.4 and the content of Appendix A.3. I thank for his permission to include this material here.

It is a pleasure to thank W. Fulton for his many years' encouragement given to my work and for informing me about an argument reproduced in Addenda to [DC-P] in the last Appendix.

The material surveyed in Section 6 stems mainly from a recent series of papers written in collaboration with J. Ratajski.

I thank also I. G. Macdonald for pointing me out some errors in the previous version.

1. Polynomials universally supported on degeneracy loci. This section summarizes mainly a series of results from [P1-5], [P-P1,2] and [P-R1].

Let $\operatorname{Mat}_{m \times n}(K)$ be the affine space of $m \times n$ matrices over a field $K$. The subvariety $D_{r}$ of $\operatorname{Mat}_{m \times n}(K)$ consisting of all matrices of rank $\leqslant r$ is called a determinantal variety (of order $r$ ). Algebro-geometric properties of these varieties were widely investigated in the seventies and eighties. The prototype of the results of this section is, however, an older result — a formula of Giambelli [G3] (1903) (see also [G1] and [G4]) for the degree of the projective determinantal variety (i.e. the class of $D_{r} \backslash\{0\}$ in $\mathbb{P}\left(\mathrm{Mat}_{m \times n}(\mathbb{C})\right)$ ). In order to perform his computations Giambelli used the machinery of symmetric polynomials developed mainly by the 18th- and 19th-century elimination theory.

Determinantal varieties are a particular case of degeneracy loci

$$
D_{r}(\varphi)=\{x \in X \mid \operatorname{rank} \varphi(x) \leqslant r\}
$$

$r=0,1,2, \ldots$ associated with a homomorphism $\varphi: F \rightarrow E$ of vector bundles on algebraic (or differentiable) variety $X$. This concept overlaps many interesting situations like varieties of special divisors (called also Brill-Noether loci) in Jacobians, Thom-Boardmann singularities, variations of Hodge structures in families of Riemann surfaces — just to mention a few; for more details and examples consult [Tu].

One of the fundamental problems in the study of concrete subscheme $D$ of a given (smooth) scheme $X$ is the computation of its fundamental class in terms of given generators of the cohomology or Chow ring of $X$. For instance, Giambelli's formula mentioned above gives the fundamental class of the (projective) determinantal variety in 
$H^{*}\left(\mathbb{P}\left(\operatorname{Mat}_{m \times n}(\mathbb{C})\right), \mathbb{Z}\right)\left({ }^{3}\right)$. In 1957 R. Thom $([\mathrm{T}])$ proved that for sufficiently general homomorphisms $\varphi: F \rightarrow E$, there exists a polynomial, depending solely on the Chern classes $c_{i}(E), c_{j}(F)$ of $E$ and $F$, which describes the fundamental class of $D_{r}(\varphi)\left(^{4}\right)$. This polynomial has been found subsequently by Porteous:

$$
\operatorname{Det}\left[c_{n-r-p+q}(E-F)\right]_{1 \leqslant p, q \leqslant m-r}
$$

where $c_{k}(E-F)$ is defined by:

$1+c_{1}(E-F)+c_{2}(E-F)+\cdots=\left(1+c_{1}(E)+c_{2}(E)+\ldots\right) /\left(1+c_{1}(F)+c_{2}(F)+\ldots\right)$.

Different variants and generalizations of $(*)$ were considered later in $[\mathrm{K}-\mathrm{L}],[\mathrm{L} 1]$, $[\mathrm{J}-\mathrm{L}-\mathrm{P}],[\mathrm{H}-\mathrm{T} 1],[\mathrm{P} 5]$ and recently in $[\mathrm{F} 2]$ (compare Section 3). In particular, note that $\left.{ }^{*}\right)$ can be rewritten using the Segre classes of $E$ and $F$ as the determinant:

$$
\operatorname{Det}\left[s_{m-r-p+q}(E-F)\right]_{1 \leqslant p, q \leqslant n-r}
$$

where $s_{k}(E-F)$ is defined by:

$1+s_{1}(E-F)+s_{2}(E-F)+\cdots=\left(1+s_{1}(E)+s_{2}(E)+\ldots\right) /\left(1+s_{1}(F)+s_{2}(F)+\ldots\right)$.

and $s_{k}(E)$ is here the $k$-th complete symmetric polynomial ([M1]) in the Chern roots of $E$. (Note that this definition differs by a sign from that for the Segre class of a bundle, used in [F1].)

Today's formulation of the Giambelli-Thom-Porteous formula uses much weaker assumptions than the transversality conditions in $[\mathrm{Po}]$ thanks to the work of Kempf and Laksov [K-L] and Fulton-MacPherson's intersection theory [F1]. (We refer the reader to [F1] for the notions of algebraic geometry used in the present article.)

TheOREM 1.0. If $X$ is a pure-dimensional Cohen-Macaulay scheme and the degeneracy locus $D_{r}(\varphi)$, endowed with the scheme structure defined by the ideal generated by $r+1$ minors of $\varphi$, is of pure codimension $(m-r)(n-r)$ in $X$ or empty, then

$$
\left[D_{r}(\varphi)\right]=\operatorname{Det}\left[s_{m-r-p+q}(E-F)\right]_{1 \leqslant p, q \leqslant n-r} \cap[X] .
$$

(In a modern treatment of intersection theory of [F1], one constructs, for every vector bundle homomorphism $\varphi$ over a pure-dimensional scheme $X$, a degeneracy class $\mathbb{D}_{r}(\varphi) \in$ $A_{\operatorname{dim} X-(m-r)(n-r)}\left(D_{r}(\varphi)\right)$ whose image in $A_{*}(X)$ is given by the right-hand side of the formula of the theorem. If $D_{r}(\varphi)$ is of pure codimension $(m-r)(n-r)$ then $\mathbb{D}_{r}(\varphi)$ is a

$\left({ }^{3}\right)$ More precisely, Giambelli calculated the degree of $D_{r}(\varphi)$ for a general map $\varphi: \mathcal{O}\left(m_{1}\right) \oplus$ $\mathcal{O}\left(m_{2}\right) \oplus \cdots \rightarrow \mathcal{O}\left(n_{1}\right) \oplus \mathcal{O}\left(n_{2}\right) \oplus \ldots$. His expression, in the notation introduced a bit later, is $\sum s_{I}(E) \cdot s \bar{I}_{\bar{I}}\left(F^{\vee}\right)$ where the sum is taken over all partitions $I$ whose diagram is contained in the rectangle $(n-r) \times(m-r)$ and the diagram of the partition $\bar{I}$ complements the one of $I^{\sim}$ in the rectangle $(m-r) \times(n-r)$; in today's language, explained in the sequel, this expression equals $s_{(m-n)^{n-r}}(E-F)$. We refer the reader to the article by D. Laksov [La] about Giambelli's work and life. This article contains also a complete bibliography of Giambelli overlapping his work on degeneracy loci formulas. Perhaps it is worth mentioning that several of Giambelli's formulas have been recently rediscovered using the Gröbner bases technique — see e.g. [He-T].

$\left({ }^{4}\right)$ As Lascoux points out, there is a little step, by combining this result of Thom and the above mentioned computation of Giambelli, to arrive at the formula from Theorem 1.0. 
positive cycle whose support is $D_{r}(\varphi)$; if, moreover $X$ is Cohen-Macaulay then $D_{r}(\varphi)$ is also Cohen-Macaulay and $\left.\mathbb{D}_{r}=\left[D_{r}(\varphi)\right].\right)\left({ }^{5}\right)$

The second domain of research concerning nonsingular degeneracy loci in nonsingular ambient spaces, is the calculation of their Chern numbers (see [H], [Na] and [H-T2]). Here, one deals with complex varieties and the problem is to find expressions depending solely on $c_{i}(E), c_{j}(F)$ and $c_{k}(T X)$ for such numbers. A natural extension of this question is to ask about similar universal formulas for the topological Euler-Poincaré characteristic of $D_{r}(\varphi)$, or, even more, for the Chern-Schwartz-MacPherson classes of these varieties, now without the smoothness assumption on $X$ and $D_{r}(\varphi)$.

Finally, the third kind of problems stems from a study of different type homology of degeneracy loci (compare [Tu]).

It turns out that all these questions are closely related with the following problem whose investigation started with the author's papers $[\mathrm{P} 1,3]$.

Problem. Which polynomials in the Chern classes of $E$ and $F$ are universally supported on the $r$-th degeneracy locus?

To state this problem precisely, assume that a homology theory $H(-)$ is given which is a covariant functor for proper morphisms and is endowed with Chern classes associated with vector bundles on a given variety $X$, acting as operators on $H(X)$. Then also the polynomials in the Chern classes of vector bundles act as operators on $H(X)$. For example, the Chow homology, the singular homology and the Borel-Moore homology have these properties (see [P-R1] for more on that).

Let $\iota_{r}: D_{r}(\varphi) \rightarrow X$ be the inclusion and let $\left(\iota_{r}\right)_{*}: H\left(D_{r}(\varphi)\right) \rightarrow H(X)$ be the induced morphism of the homology groups. Fix integers $m, n>0$ and $r \geqslant 0$. Introduce $m+n$ variables $c_{1}, \ldots, c_{n} ; c_{1}^{\prime}, \ldots, c_{m}^{\prime}$ such that $\operatorname{deg}\left(c_{i}\right)=\operatorname{deg}\left(c_{i}^{\prime}\right)=i$. Let $\mathbb{Z}\left[c ., c^{\prime}.\right]=$ $\mathbb{Z}\left[c_{1}, \ldots, c_{n}, c_{1}^{\prime}, \ldots, c_{m}^{\prime}\right]$ be the polynomial algebra. Following $[\mathrm{P} 1,3]$ we say that $P \in$ $\mathbb{Z}\left[c ., c^{\prime}.\right]$ is universally supported on the $r$-th degeneracy locus if

$$
P\left(c_{1}(E), \ldots, c_{n}(E), c_{1}(F), \ldots, c_{m}(F)\right) \cap \alpha \in \operatorname{Im}\left(\iota_{r}\right)_{*}
$$

for any homomorphism $\varphi: F \rightarrow E$ of vector bundles on $X$ such that $n=\operatorname{rank} E$, $m=\operatorname{rank} F$ and any $\alpha \in H(X)$. Denote by $\mathcal{P}_{r}$ the set (which is, in fact, an ideal) of all polynomials universally supported on the $r$-th degeneracy locus. Of course, the Giambelli-Thom-Porteous polynomials (*) describing $D_{i}(\varphi)$ for $i \leqslant r$ belong to $\mathcal{P}_{r}$, but they do not generate this ideal if $r \geqslant 1$. An analogous problem can be stated for symmetric

$\left(^{5}\right)$ Recall that if $D \subset X$ is a (closed) subscheme then $[D] \in A_{*}(X)$ is the class of the fundamental cycle associated with $D$, i.e., if $D=D_{1} \cup \ldots \cup D_{n}$ is a minimal decomposition into irreducible components then

$$
[D]=\sum_{i=1}^{n}\left(\operatorname{length} \mathcal{O}_{D, D_{i}}\right)\left[D_{i}\right],
$$

where $\mathcal{O}_{D, D_{i}}$ is the local ring of $D$ along $D_{i}$. Recall also that if $f: X \rightarrow Y$ is a proper morphism then it induces a morphism of abelian groups $f_{*}: A_{*}(X) \rightarrow A_{*}(Y)$ such that $f_{*}[V]=\operatorname{deg}\left(\left.f\right|_{V}\right)[f(V)]$ if $\operatorname{dim} f(V)=\operatorname{dim} V$ and 0 - otherwise. In particular, if $f$ establishes a birational isomorphism of $V$ and $f(V)$ then $f_{*}[V]=[f(V)]$. If $X$ and $Y$ are nonsingular then a morphism $f: X \rightarrow Y$ induces a ring homomorphism $f^{*}: A^{*}(Y) \rightarrow A^{*}(X)$. If $X, Y$ are possibly singular and $f$ is flat (or regular embedding) then there exists a group homomorphism $f^{*}: A_{*}(Y) \rightarrow A_{*}(X)$ 
(resp. antisymmetric) morphisms: $F=E^{\vee}, \varphi^{\vee}=\varphi$ (resp. $\varphi^{\vee}=-\varphi$ ). In this case the corresponding ideal $\mathcal{P}_{r}^{s}$ (resp. $\mathcal{P}_{r}^{a s} r$-even) is contained in $\mathbb{Z}\left[c_{1}, \ldots, c_{n}\right]=\mathbb{Z}[c$.$] .$

It follows from the "main theorem on symmetric polynomials" that for a sequence of variables $A=\left(a_{1}, \ldots, a_{n}\right)$, where $\operatorname{deg}\left(a_{i}\right)=1$, the assignment

$$
c_{i} \mapsto(i \text {-th elementary symmetric polynomial in } A)
$$

defines an isomorphism of $\mathbb{Z}[c$.$] and S \mathcal{P}(A)$ - the ring of symmetric polynomials in $A$. Similarly, by considering an analogous assignment for the $c_{j}^{\prime}$ 's and a second sequence of variables $B=\left(b_{1}, \ldots, b_{m}\right)$, we get an isomorphism of $\mathbb{Z}\left[c ., c^{\prime}.\right]$ with $S \mathcal{P}(A \mid B)=S \mathcal{P}(A) \otimes$ $S \mathcal{P}(B)$ - the ring of symmetric polynomials in $A$ and $B$ separately.

A precise description of the ideals $\mathcal{P}_{r}, \mathcal{P}_{r}^{s}$ and $\mathcal{P}_{r}^{a s}$ requires two families of symmetric polynomials.

(i) Let $I=\left(i_{1}, \ldots, i_{k}\right)$ be a sequence of integers. We define

$$
s_{I}(A-B)=\operatorname{Det}\left[s_{i_{p}-p+q}(A-B)\right]_{i \leqslant p, q \leqslant k},
$$

where $s_{i}(A-B)$ is a homogeneous polynomial of degree $i$ such that

$$
\sum_{i=-\infty}^{\infty} s_{i}(A-B)=\prod_{i=1}^{n}\left(1-a_{i}\right)^{-1} \prod_{j=1}^{m}\left(1-b_{j}\right) .
$$

Observe that the corresponding polynomials $s_{I}\left(c . / c^{\prime}.\right)$ in the variables $c$. and $c^{\prime}$. are determined by

$$
s_{i}\left(c . / c^{\prime} .\right)=s_{i}-s_{i-1} c_{1}^{\prime}+\cdots+(-1)^{i-1} s_{1} c_{i-1}^{\prime}+(-1)^{i} c_{i}^{\prime}
$$

where

$$
s_{i}=s_{i-1} c_{1}-s_{i-2} c_{2}+\ldots+(-1)^{i-2} s_{1} c_{i-1}+(-1)^{i-1} c_{i}
$$

for $i>0$ and $s_{i}=0$ for $i<0, s_{0}=1$.

Moreover, we put $s_{I}(A)=s_{I}(A-B)$ for $B=(0, \ldots, 0)$ and similarly $s_{I}(c)=$. $s_{I}\left(c . / c^{\prime}\right.$.) for $c_{j}^{\prime}=0, j=1, \ldots, m$.

(ii) Let $Q_{i}(A)$ be a symmetric polynomial defined by the expansion

$$
\sum_{i=-\infty}^{\infty} Q_{i}(A) t^{i}=\prod_{i=1}^{n}\left(1+t a_{i}\right)\left(1-t a_{i}\right)^{-1}
$$

Given nonnegative integers $i, j$, we set

$$
Q_{i, j}(A)=Q_{i}(A) Q_{j}(A)+2 \sum_{p=1}^{j}(-1)^{p} Q_{i+p}(A) Q_{j-p}(A) .
$$

Finally, if $I=\left(i_{1}, \ldots, i_{k}\right)$ is a sequence of positive integers then for odd $k$ we put

$$
Q_{I}(A)=\sum_{p=1}^{k}(-1)^{k-1} Q_{i_{p}}(A) Q_{i_{1}, \ldots, i_{p-1}, i_{p+1}, \ldots, i_{k}}(A),
$$

and for even $k$,

$$
Q_{I}(A)=\sum_{p=2}^{k}(-1)^{k} Q_{i_{1}, i_{p}}(A) Q_{i_{2}, \ldots, i_{p-1}, i_{p+1}, \ldots, i_{k}}(A) .
$$



by

Observe that the corresponding polynomials $Q_{I}(c$.$) in the variables c$. are determined

$$
Q_{i}(c .)=s_{i}+s_{i-1} c_{1}+\cdots+s_{1} c_{i-1}+c_{i}
$$

for $i>0$ and $Q_{0}(c)=1,. Q_{i}(c)=$.0 for $i<0$.

The polynomials $s_{I}(A)$ and $s_{I}(c$.) are called Schur polynomials or $S$-polynomials. The polynomials $s_{I}(A-B)$ and $s_{I}\left(c . / c^{\prime}\right.$.) are often called supersymmetric Schur polynomials - for an account to their properties we refer to $[\mathrm{P} 4]$ and $[\mathrm{P}-\mathrm{T}]$. The polynomials $Q_{I}(A)$ are called Schur Q-polynomials — for an account to their properties we refer to $[\mathrm{H}-\mathrm{H}]$ and [P4]. For another expression of $Q_{I}(A)$ in the form of a quadratic polynomial in the $s_{J}(A)$ 's, see [La-Le-T1].

Now, let $E$ and $F$ be two vector bundles on $X$. Then $s_{I}(E-F)$ is obtained from $s_{I}\left(c . / c^{\prime}.\right)$ via the specialization $c_{i}:=c_{i}(E), i=1, \ldots, n ; c_{j}^{\prime}:=c_{j}(F), j=1, \ldots, m$; and $s_{I}(E)-$ from $s_{I}(c$. $)$ by the substitution $c_{i}:=c_{i}(E)$. Similarly we define $Q_{I}(E)$ as the specialization of $Q_{I}(c$. $)$ with $c_{i}:=c_{i}(E)$.

Recall that by a partition (of $n$ ) we understand a sequence of integers $I=\left(i_{1}, \ldots, i_{k}\right.$ ), where $i_{1} \geqslant i_{2} \geqslant \cdots \geqslant i_{k} \geqslant 0$ and $\sum i_{p}=n$. A partition with strictly decreasing parts is called strict. For partitions $I, J$ we write $I \supset J$ if $i_{1} \geqslant j_{1}, i_{2} \geqslant j_{2}, \ldots$; the partition $(i, \ldots, i)(r$-times $)$ is denoted by $(i)^{r}$; finally the partition $(k, k-1, \ldots, 2,1)$ is denoted by $\rho_{k}$.

Note that for every strict partition $I=\left(i_{1}>\cdots>i_{k}>0\right)$, one has $Q_{I}(c)=.2{ }^{k} P_{I}(c$. for some polynomial $P_{I}(c$. $)$ with integer coefficients. These polynomials are called $S c h u r$ $P$-polynomials. At first, the ideals $\mathcal{P}_{r}, \mathcal{P}_{r}^{s}$ and $\mathcal{P}_{r}^{a s}$ were described for the Chow homology. Let us give first a coarse description:

Theorem $1.1[\mathrm{P} 1,3]$. Assume that $H(-)=A_{*}(-)$ is the Chow homology theory. Then

(i) The ideal $\mathcal{P}_{r}$ is generated by $s_{I}\left(c . / c^{\prime}.\right)$, where $I$ runs over all partitions $I \supset(m-r)^{n-r}$.

(ii) The ideal $\mathcal{P}_{r}^{s}$ is generated by $Q_{I}\left(\right.$ c.), where $I$ runs over all partitions $I \supset \rho_{n-r}$.

(iii) The ideal $\mathcal{P}_{r}^{a s}$ (r-even) is generated by $P_{I}(c$.$) , where I$ runs over all partitions $I \supset \rho_{n-r}$.

Observe that the "positive" generator of the ideal $\mathcal{P}_{r}$ agrees with the Segre class version of the Giambelli-Thom-Porteous polynomial from Theorem 1.0. The analogous generators of the ideals $\mathcal{P}_{r}^{s}$ and $\mathcal{P}_{r}^{a s}$ are of different (Pfaffian) form than the determinantal expressions given in $[\mathrm{J}-\mathrm{L}-\mathrm{P}],[\mathrm{H}-\mathrm{T} 1]$ and $[\mathrm{P} 5]$.

To prove that the quoted polynomials belong to $\mathcal{P}_{r}, \mathcal{P}_{r}^{s}$ and $\mathcal{P}_{r}^{a s}$, the key tools are certain factorization formulas and formulas for the Gysin map for Grassmannian bundles.

In the sequel, having two partitions $I$ and $J$ with $l(I) \leqslant k$ and $l\left(J^{\sim}\right) \leqslant i\left({ }^{6}\right)$, by $(i)^{k}+I, J$ we denote the partition $\left(i+i_{1}, \ldots, i+i_{k}, j_{1}, j_{2}, \ldots\right)$.

$\left({ }^{6}\right)$ For a given partition $I, l(I)=\operatorname{card}\left\{p: i_{p}>0\right\}$ denotes its length and $I^{\sim}$ denotes the partition conjugate to $I$, i.e., $\left(h_{1}, h_{2}, \ldots\right)$ where $h_{p}=\operatorname{card}\left\{q: i_{q} \geqslant p\right\}$. 
Proposition 1.2 (Factorization Formula). Let $I, J$ be two partitions such that $l(I) \leqslant$ $n$ and $l\left(J^{\sim}\right) \leqslant m$. Then $\left({ }^{7}\right)$

$$
\begin{aligned}
s_{(m)^{n}+I, J}(A-B) & =s_{I}(A) s_{(m)^{n}}(A-B) s_{J}(-B) \\
& =(-1)^{|J|} s_{I}(c .) s_{(m)^{n}}\left(c . / c .^{\prime}\right) s_{J} \sim\left(c .^{\prime}\right) \\
Q_{\rho_{n-1}+I}(A) & =Q_{\rho_{n-1}}(A) s_{I}(A) .
\end{aligned}
$$

(Formula (ii) is due to Stanley; for the history of (i) we refer to [L3]. Both the formulas are just special instances of much more general identities for which we refer the interested reader to $[\mathrm{P} 4,1.3],[\mathrm{P}-\mathrm{T}]$ and $[\mathrm{La}-\mathrm{Le}-\mathrm{T} 1]$.

Proposition 1.3. Let $\pi=\pi_{E}: \mathcal{G}=G^{q}(E) \rightarrow X$ be the Grassmannian bundle parametrizing q-quotients of E. Write $r=n-q$. Let

$$
0 \leftarrow Q=Q_{E} \leftarrow E_{\mathcal{G}} \leftarrow R=R_{E} \leftarrow 0
$$

be the tautological exact sequence of vector bundles on $\mathcal{G}$. Let $\alpha \in A_{*}(X)$.

(i) $[\mathrm{J}-\mathrm{L}-\mathrm{P}],[\mathrm{P} 3]$. For every vector bundle $F$ on $X$ and any sequences of integers $I=$ $\left(i_{1}, \ldots, i_{q}\right), J=\left(j_{1}, \ldots, j_{r}\right)$,

$$
\left(\pi_{E}\right)_{*}\left[s_{I}\left(Q-F_{\mathcal{G}}\right) s_{J}\left(R-F_{\mathcal{G}}\right) \cap \pi_{E}^{*} \alpha\right]=s_{i_{1}-r, \ldots, i_{q}-r, j_{1}, \ldots, j_{r}}(E-F) \cap \alpha,
$$

(ii) Let $I=\left(i_{1}, \ldots, i_{k}\right), J=\left(j_{1}, \ldots, j_{h}\right)$ be two sequences of positive integers, $k \leqslant q$, $h \leqslant r$. Then

$$
\left(\pi_{E}\right)_{*}\left[c_{\mathrm{top}}(Q \otimes R) P_{I} Q P_{J} R \cap \pi_{E}^{*} \alpha\right]=d P_{I, J}(E) \cap \alpha,
$$

where $d$ is zero if $(q-k)(r-h)$ is odd, and $(-1)^{(q-k) r}\left(\begin{array}{c}{[(n-k-h) / 2]} \\ {[(q-k) / 2]}\end{array}\right)$ - otherwise $\left(^{8}\right)$.

For a proof of (ii), see Appendix A.1.

Propositions 1.2 and 1.3 allow one to prove the following algebraic result providing finite sets of generators of the ideals in question.

Proposition 1.4 [P3].

(i) $\left(s_{I}\left(c . / c^{\prime}.\right), I \supset(m-r)^{n-r}\right)=\left(s_{(m-r)^{n-r}+I}\left(c . / c^{\prime}.\right), I \subset(r)^{n-r}\right)$,

(ii) $\left(Q_{I}(c),. I \supset \rho_{n-r}\right)=\left(Q_{\rho_{n-r}+I}(c),. I \subset(r)^{n-r}\right)$,

(iii) $\left(P_{I}(c),. I \supset \rho_{n-r-1}\right)=\left(P_{\rho_{n-r-1}+I}(c),. I \subset(r)^{n-r}\right), r-$ even.

- thus these ideals are generated by $\left(\begin{array}{l}n \\ r\end{array}\right)$ elements.

Note that it is still an open problem to show that these sets form minimal sets of generators of the corresponding ideals (in case (i), we assume that $m \geqslant n$ ).

For an explicit $\mathbb{Z}$-basis of the ideal in (i), see [P3, Proposition 6.2]. It would be valuable to have a similar result for the ideals in (ii) and (iii). Moreover, the ideal in (i) is prime $([\mathrm{P} 2,4])$, and is a set-theoretical complete intersection (is equal to the radical of an ideal generated by a regular sequence of length $r+1$ (loc.cit.)).

$\left({ }^{7}\right)$ For a given partition $I$, we write $|I|:=\sum i_{p}$ — the sum of parts of $I$, i.e. the number partitioned by $I$.

$\left.{ }^{8}\right) I, J$ denotes here and in Appendix A.1 the juxtaposition of $I$ and $J$. 
As shown in loc.cit., the ideal in (i) gives a generalization of the resultant of two polynomials in one variable. Let

$$
A(x)=x^{n}+\sum_{i=1}^{n} c_{i} x^{n-i}, \quad B(x)=x^{m}+\sum_{j=1}^{m} c_{j}^{\prime} x^{m-j}
$$

be two polynomials in one variable with generic coefficients. It follows from the classical algebra, that there exists a polynomial in $\left\{c_{i}\right\},\left\{c_{i}^{\prime}\right\}$ called the resultant, whose vanishing (after a specialization of $\left\{c_{i}\right\},\left\{c_{i}^{\prime}\right\}$ to an algebraically closed field) implies that the corresponding polynomials have a common root (see, e.g., [L3] for an approach to the resultant via the symmetric polynomials).

Now, let $\mathcal{T}_{r}$ be the ideal of all $P \in \mathbb{Z}\left[c ., c^{\prime}.\right]$, which vanish if, after a specialization of $\left\{c_{i}\right\},\left\{c_{i}^{\prime}\right\}$ to a field, $A(x)$ and $B(x)$ have $r+1$ common roots. Surprisingly (or not) we have

TheOREM $1.5[\mathrm{P} 2,4] . \mathcal{T}_{r}=\left(s_{(m-r)^{n-r}+I}\left(c . / c^{\prime}.\right), I \subset(r)^{n-r}\right)$.

In other words $\mathcal{T}_{r}=\mathcal{P}_{r}$ in the above notation. It would be interesting to have an intrinsic proof of this equality. It is shown in $[\mathrm{L}-\mathrm{P}]$ that an analogous ideal defined in the ring $\mathbb{Z}[A B]$ of all polynomials in $A$ and $B$ is just generated by $\mathcal{T}_{r} \subset S \mathcal{P}(A \mid B) \subset \mathbb{Z}[A B]$. A similar interpretation is given in [P4] (and correspondingly in [L-P]) for the ideals $\mathcal{P}_{r}^{s}$ and $\mathcal{P}_{r}^{a s}$ generated by $Q$ - and $P$-polynomials respectively.

Let us come back to Theorem 1.1. The proof that the ideal $\mathcal{P}_{r}$ is actually generated by the above polynomials is based on the investigation of the tautological determinantal variety $\underline{D}_{r} \subset \underline{\operatorname{Hom}}(F, E)$ (the fibre of $\underline{D}_{r}$ over a point $x \in X$ is equal to $\{f \in \operatorname{Hom}(F(x), E(x)) \mid \operatorname{rank}(f) \leqslant r\})$. The bundles $E$ and $F$ occurring in this construction are some "universal enough" vector bundles over the product $G G$ of two Grassmannians (see [P3]). In fact, in [P3], two proofs of this assertion are given. One of them [P3, pp. 441-445] is by induction on $r$ with the help of an exact sequence of Chow groups

$$
A_{*}\left(\underline{D}_{r-1}\right) \rightarrow A_{*}\left(\underline{D}_{r}\right) \rightarrow A_{*}\left(\underline{D}_{r} \backslash \underline{D}_{r-1}\right) \rightarrow 0
$$

and a detailed analysis of $A_{*}\left(\underline{D}_{r} \backslash \underline{D}_{r-1}\right)$. The second one [P3, pp. 428-432] uses a certain desingularization of $\underline{D}_{r}$ and has been ameliorated in [P-R1] to give the assertion also for the Borel-Moore homology and the singular homology.

TheOREM 1.6 [P-R1]. The statement of Theorem 1.1 is true also for $H(-)$ being the Borel-Moore homology (both, the classical one and that defined by Laumon in characteristic $p$ ) as well as for the singular homology (with integer coefficients).

Since the same applies to Proposition 1.3, when appropriately formulated, the proof that the quoted polynomials belong to $\mathcal{P}_{r}$ is the same.

On the other hand, the proof that the ideal $\mathcal{P}_{r}$ is generated by the above polynomials uses the following compactification of $\underline{D}_{r}$. Let us embed the above $\underline{\operatorname{Hom}}(F, E)$ into a Grassmannian bundle $\mathbf{X}=G_{m}(F \oplus E)$ by assigning fibrewise to $f \in \overline{\operatorname{Hom}}(F(x), E(x))$ its (graph of $f$ ) $\in G_{m}(F(x) \oplus E(x)), x$ belonging to the base space $G G$. On $\mathbf{X}$ there exists a natural tautological extension of the universal homomorphism on $\underline{\operatorname{Hom}}(F, E)$ and its degeneracy loci serve to prove the assertion.

An important advantage of the above compactification as well as a certain natural desingularization $\mathbf{Z}$ of it is the vanishing of their odd homology groups - this is not the case of $\underline{D}_{r}$ and its analogous desingularization (see [P-R1]). Here $\mathbf{Z}$ is the subscheme 
of zeros of the homomorphism $F_{\mathbf{G}} \rightarrow E_{\mathbf{G}} \rightarrow Q$ on $\mathbf{G}=G_{r}(E)$ where the first map is the pullback to $\mathbf{G}$ of $\varphi$. Let $\eta: \mathbf{Z} \rightarrow \mathbf{D}_{r}$ be the restriction of $\pi: \mathbf{G} \rightarrow X$ to $\mathbf{Z}$, and let $j$ be the closed immersion of $\mathbf{Z}$ into $\mathbf{G}$. Then by using the rank-stratification $\left\{\mathbf{D}_{k} \backslash \mathbf{D}_{k-1}\right\}$ of $\mathbf{D}_{r}$, the induced stratification $\mathbf{Z}^{k} \backslash \mathbf{Z}^{k-1}$ of $\mathbf{Z}\left(\mathbf{Z}^{k}=\eta^{-1} \mathbf{D}_{k}\right)$, and proving (for the Borel-Moore homology) that $\mathrm{cl}_{\mathbf{D}_{k}}$ and $\mathrm{cl}_{\mathbf{Z}^{k}}$ are isomorphisms, one shows that the induced push-forward map $\eta_{*}: H(\mathbf{Z}) \rightarrow H\left(\mathbf{D}_{r}\right)$ is surjective. Also, by analyzing the geometry of $\mathbf{Z}$, one shows that $j^{*}$ is surjective. This implies, by the projection formula, that $\operatorname{Im} j_{*}$ is a principal ideal in $H(\mathbf{G})$ generated by the fundamental class $[\mathbf{Z}]$. It follows then, from the commutative diagram

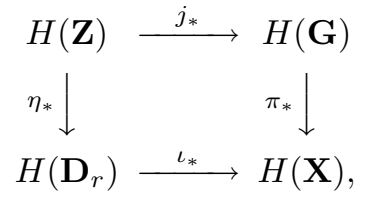

that $\operatorname{Im} \iota_{*}=\pi_{*}([\mathbf{Z}] H(\mathbf{G}))$. This identity together with some algebra of symmetric polynomials (which allows one to express explicitly $[\mathbf{Z}] H(\mathbf{G})$ ) yields the desired assertion about $\operatorname{Im} \iota_{*}$. In this way we obtain a proof which is valid both for Chow homology and other homology theories simultaneously.

In a similar way, though overcoming some additional difficulties, one can prove the analogous theorem in the symmetric and antisymmetric cases.

Theorem 1.1 allows us to calculate the Chow groups of some degeneracy loci. A prototype of these results is the following result from [B]. Let $R$ be a normal noetherian ring, $M$ - a $m \times n$ matrix of indeterminates, $\mathcal{I}$ - the ideal generated by all $(r+1)$-minors of $M$. Then, the divisor class groups satisfy: $\mathrm{Cl}(R[M] / \mathcal{I}) \cong \mathrm{Cl}(R) \oplus \mathbb{Z}$.

The geometric analogue of $\mathrm{Cl}$ is $A^{1}$ (the Chow group of codimension 1 cycles modulo rational equivalence). Keeping the above notation for the tautological degeneracy loci in Hom-bundle one has $\left(A^{i}(-)\right.$ denotes below the Chow group of codimension $i$ algebraic cycles modulo rational equivalence):

THEOREM 1.7 [P3]. If $m \geqslant n$ then the Chow group of $\underline{D}_{r}$ is canonically isomorphic to the Chow group of $G_{r}(E)$. Therefore, for every $i, A^{i}\left(\underline{D}_{r}\right)=\bigoplus A^{i-|I|}(X)$, the sum over all partitions $I \subset(r)^{n-r},|I| \leqslant i$.

Let $\operatorname{Mat}_{m \times n}(K)$ denote the affine space of $m \times n$ matrices over a field $K$ (assume $m \geqslant$ $n$ without loss of generality) and $D_{r} \subset \operatorname{Mat}_{m \times n}(K)$ be the subscheme defined by the ideal generated by all minors of order $r+1$. The theorem implies, in particular, that for every $K$-scheme $X, A^{i}\left(X \times D_{r}\right) \cong \bigoplus A^{i-|I|}(X)$, the sum as above. For $i=1$ this is a geometric analogue of the result from [B]. Note that the Chow group of $D_{r}$ is isomorphic to the Chow group of the Grassmannian $G_{r}\left(K^{n}\right)$. This could create an impression that homologically $D_{r}$ behave like spaces which admit a cellular decomposition. This is, however, not the case - see [P-R1] where it is shown that complex determinantal varieties have nontrivial Borel-Moore homology groups of odd degree.

It would be interesting to find analogues of Theorem 1.7 for the tautological degeneracy loci of homomorphisms with symmetries.

Finally we pass to perhaps the most spectacular application of the theory of polynomials universally supported on degeneracy loci. This is a formula for the Chern-SchwartzMacPherson classes of degeneracy loci associated with an $r$-general vector bundle ho- 
momorphism $\varphi: F \rightarrow E$ over a (possibly singular) complex analytic variety $X$. The Chern-Schwartz-MacPherson class $c_{*}(X)$ of a variety $X$ has its value in the Borel-Moore homology of $X$ and satisfies similar properties as the Chern class $c(T X)$ of the tangent bundle of a complex manifold $X$. In particular, for a possibly singular compact analytic variety $X$, we have the following expression for the topological Euler-Poincaré characteristic:

$$
\chi(X)=\int_{X} c_{*}(X)
$$

(see, for instance [F1, Chap. 19]). Let us now fix a Whitney stratification $\mathcal{X}$ of $X$. Let $E$ be a holomorphic vector bundle on $X$ and $Z$ - the variety of zeros of a holomorphic section $s$ of $E$. Assume that $s$ intersects, on each stratum of $\mathcal{X}$, the zero section of $E$ transversely. Let $\iota: Z \rightarrow X$ be the inclusion.

LEMMA 1.8 [P-P2].

$$
\iota_{*}\left(c_{*}(Z)\right)=c(E)^{-1} \cdot c_{\mathrm{top}}(E) \cap c_{*}(X) .
$$

In particular, for a compact analytic variety $X$,

$$
\chi(Z)=\int_{X} c(E)^{-1} \cdot c_{\mathrm{top}}(E) \cap c_{*}(X) .
$$

This is the simplest instance of the formula in question. To state the result in the most general form we need a notion of $r$-generality of a vector bundle homomorphism. We say that $\varphi$ is $r$-general if the section $s_{\varphi}: X \rightarrow \underline{\operatorname{Hom}}(F, E)$ induced by $\varphi$ intersects, on each stratum of the Whitney stratification $\mathcal{X}$, the subset $\underline{D}_{k} \backslash \underline{D}_{k-1}$ transversely for every $k=0,1, \ldots, r$. For a pure-dimensional nonsingular $X$, this condition can be expressed in a more transparent way: a morphism $\varphi$ is $r$-general iff for every $k=0,1, \ldots, r$, the subset $D_{k}(\varphi) \backslash D_{k-1}(\varphi)$ is nonsingular of pure dimension $\operatorname{dim} X-(m-k)(n-k)$ (here, $\left.D_{-1}(\varphi)=\emptyset\right)$.

Let $m \wedge n$ denote the minimum of $m$ and $n$. We now define the following element in $H_{*}(X)$. We set

where

$$
\Psi(k):=P_{k}(E, F) \cap c_{*}(X),
$$

$$
P_{k}(E, F):=\sum(-1)^{|I|+|J|} D_{I, J}^{m-k, n-k} s_{(m-k)^{n-k}+I, J \sim}(E-F) .
$$

Here, the sum is over all partitions $I, J$ such that $l(I) \leqslant m \wedge n-k, l(J) \leqslant m \wedge n-k$, and the numbers $D_{I, J}^{m-k, n-k}$ are some binomial determinants which will be defined in Theorem 2.4(i).

The following formula gives an explicit expression for the image of the Chern-SchwartzMacPherson class of $D_{r}(\varphi)$ in the homology of $X$. Recall that $\iota: D_{r}(\varphi) \rightarrow X$ denotes the inclusion.

THEOREM 1.9 [P-P1,2]. If $\varphi$ is $r$-general then one has in $H_{*}(X)$

$$
\iota_{*}\left(c_{*}\left(D_{r}(\varphi)\right)\right)=\sum_{k=0}^{r}(-1)^{k}\left(\begin{array}{c}
m \wedge n-r+k-1 \\
k
\end{array}\right) \Psi(r-k) .
$$

In particular, if $X$ is a compact analytic variety, then

$$
\chi\left(D_{r}(\varphi)\right)=\int_{X} \sum_{k=0}^{r}(-1)^{k}\left(\begin{array}{c}
m \wedge n-r+k-1 \\
k
\end{array}\right) \Psi(r-k) .
$$


Under the assumption $D_{r-1}(\varphi)=\emptyset$, the above formula reads $\chi\left(D_{r}(\varphi)\right)=\int_{X} \Psi(r)$. This result was established earlier in [P3] as a particular case of an algorithm for computation the Chern numbers of nonsingular degeneracy loci.

The essence of the argument is to pass first to the above described desingularization of $D_{r}(\varphi)$ and calculate explicitly the image of the homology dual to its Chern class in the homology of $X$. To this end, by using some algebra (of symmetric polynomials and Gysin maps), we show that this image has the form $P \cap c_{*}(X)$ where $P=P\left(\left\{c_{i}\right\},\left\{c_{j}^{\prime}\right\}\right)$ is a polynomial universally supported on the $r$-th degeneracy locus and not universally supported on the $(r-1)$-th one, specialized by setting $c_{i}=c_{i}(E), c_{j}^{\prime}=c_{j}(F)$. Thus "morally", without changing the result of the computation, we can assume that $D_{r-1}(\varphi)=\emptyset$. But then the desingularization equals $D_{r}(\varphi)$ and the wanted class is known by the result of [P3] quoted above.

Secondly, stratifying $D_{r}(\varphi)$ by the subsets where the rank of $\varphi$ is constant, the desingularization turns out to be a Grassmannian bundle over each stratum. This leads to an equation with the known $H_{*}(X)$-image of the Chern class of the desingularization on the one side and a linear combination of the unknown $H_{*}(X)$-images of the Chern-SchwartzMacPherson classes of $D_{k}(\varphi) \quad(k \leqslant r)$ — on the other. By varying $r$, this leads to a system of linear equations in the unknown $H_{*}(X)$-images of the Chern-Schwartz-MacPherson classes of $D_{r}(\varphi)$ (and with known coefficients). Solving this system of equations with the help of some algebra of binomial numbers, one gets the formula looked at.

As a by-product of our considerations, we also get a formula for the Intersection Homology-Euler characteristic of $D_{r}(\varphi)$ associated with an $r$-general morphism $\varphi$ :

THEOREM 1.10 [P-P2]. If $X$ is nonsingular compact analytic variety and $\varphi$ is $r$-general, then

$$
\chi_{I H}\left(D_{r}(\varphi)\right)=\int_{X} \Psi(r) .
$$

As an example of application of Theorem 1.9, we provide an expression for the topological Euler-Poincaré characteristic of the Brill-Noether loci $W_{d}^{r}(C)=\left\{L \in \operatorname{Pic}^{d}(C) \mid\right.$ $\left.h^{0}(C, L)>r\right\}$ parametrizing all complete linear series of degree $d$ and dimension $r$ on a general curve $C$ of genus $g$. Let $\rho:=\rho(r):=\rho(g, d, r):=g-(r+1)(g-d+r)$ be the Brill-Noether number.

For $\rho(r) \geqslant 0$, let

$$
\Phi(g, d, r)=(-1)^{\rho(r)} g ! \sum D_{I, J}^{r+1, g-d+r} / h\left(I_{g, d, r}+I, J^{\sim}\right),
$$

where $I_{g, d, r}$ is the partition $(r+1)^{g-d+r}$, the sum is over partitions $I, J$ with length $\leqslant(r+1) \wedge(g-d+r)$ and such that $|I|+|J|=\rho(r)$. Moreover, for a partition $I, h(I)$ denotes the product of all hook lengths associated with the boxes in the Ferrers' diagram of $I$ (see [M1, Chap. I]). We set $\Phi(g, d, r)=0$ if $\rho(r)<0$.

TheOrem 1.11 [P-P2]. Assume that a curve $C$ of genus $g$ is general. Let $d, r$ be integers as above and such that $\rho(r) \geqslant 0$. Then one has

$$
\chi\left(W_{d}^{r}(C)\right)=\sum_{k \geqslant r}(-1)^{k-r}\left(\begin{array}{c}
k \\
k-r
\end{array}\right) \Phi(g, d, k) .
$$

From this formula, one deduces the following corollary. If we fix $g, d, r$ such that $\rho(r) \geqslant 0$ and the nonnegative numbers $\rho(r), \rho(r+1), \ldots$ change successively the parity, then $\chi\left(W_{d}^{r}(C)\right)>0$ (resp. $\left.\chi\left(W_{d}^{r}(C)\right)<0\right)$ iff $\rho(r)$ is even (resp. $\rho(r)$ is odd). Observe 
that the above numbers change successively the parity if $r+1$ and $r+g-d$ are of the same parity. The latter condition holds iff $g \not \equiv d(\bmod 2)$. Thus we get, in the situation of the theorem, the following result.

Corollary 1.12 [P-P2]. Assume $g \not \equiv d(\bmod 2)$. Then one has $\chi\left(W_{d}^{r}(C)\right)<0$ (resp. $\left.\chi\left(W_{d}^{r}(C)\right)>0\right)$ iff $g \equiv r(\bmod 2) \quad($ resp. $g \not \equiv r(\bmod 2))$.

For example, if $\rho=0$, we have

$$
\Phi(g, d, r)=\operatorname{card}\left(W_{d}^{r}(C)\right)=g ! / h\left((r+1)^{g-d+r}\right),
$$

which is the classical Castelnuovo formula, expressed here using the hook number of $I_{g, d, r}$.

2. Some explicit formulas for Chern and Segre classes of tensor bundles with applications to enumerative geometry. In this paper, by $S^{I} E$ we will denote the Schur bundle associated with a bundle $E$ and partition $I$ (whenever we speak about Schur bundles, we assume, for simplicity, that the ground field is of characteristic zero). Recall that if $|I|=n$ and if $S_{n}$ stands for the symmetric group with $n$ ! elements, then $S^{I} E=\operatorname{Hom}_{S_{n}}\left(\Sigma^{I}, E^{\otimes n}\right)$ where $\Sigma^{I}$ is the corresponding irreducible representation of the group $S_{n}$ and this group acts on $E^{\otimes n}$ via the permutations of the factors. Thus in particular $S^{(n)} E=S_{n}(E)$, the $n$-th symmetric power; and $S^{(1)^{n}} E=\Lambda^{n}(E)$, the $n$-th exterior power. In other words, $S^{I} E$ is the tensor bundle of $E$ associated with the irreducible representation of $G L_{n}$ defined by $I$.

The problem of determining the Schur polynomials decomposition of $s_{I}\left(S^{J} E\right)$ is very far of being solved. The present section and Section 7 provide some partial information related to this question.

Throughout this paper, for a vector bundle $E$, we write $c_{\text {top }}(E)$ instead of $c_{\text {rank } E}(E)$. We show first that the Schur polynomials decomposition of $c_{\text {top }}\left(S^{J} E\right)$ determines the one of $c\left(S^{J} E\right)$.

Proposition 2.1. If $c_{\mathrm{top}}\left(S^{J} E\right)=\sum_{K} m_{K} s_{K}(E)$, the sum over partitions $K$, then

$$
c\left(S^{J} E\right)=|J|^{-\operatorname{rank}\left(S^{J} E\right)} \sum_{K} \sum_{L \subset K}|J|^{|L|} m_{K} d_{K L} s_{L}(E) .
$$

where the sum is over partitions $K=\left(k_{1}, \ldots, k_{n}\right), L=\left(l_{1}, \ldots, l_{n}\right)$, where $n=$ rank $E$, and

$$
d_{K L}=\operatorname{Det}\left[\left(\begin{array}{c}
k_{p}+n-p \\
l_{q}+n-q
\end{array}\right)\right]_{1 \leqslant p, q \leqslant n} .
$$

For a proof see Appendix A.2.

(In particular, note that if $c_{\mathrm{top}}\left(S^{J} E\right)$ and the Segre classes $s_{i}\left(S^{J} E\right), i \leqslant p$, are known, then the remaining Segre classes $s_{i}\left(S^{J} E\right), i>p$, are also determined.)

Recall that the Schur polynomials decompositions of $c\left(S^{2} E\right)$ and $c\left(\Lambda^{2}(E)\right)$ are known.

Proposition 2.2 [L2]. If $\operatorname{rank} E=n$ then

$$
c_{\text {top }}\left(S^{2} E\right)=2^{n} s_{\rho_{n}}(E) \quad \text { and } \quad c_{\text {top }}\left(\Lambda^{2}(E)\right)=s_{\rho_{n-1}}(E) .
$$

(Note that [L2] also contains a formula for the decomposition of $c(E \otimes F)$ into Schur polynomials.) 
EXAMPLE 2.3. In the following, $s_{I}=s_{I}(E)$.

If $\operatorname{rank} E=4$ then

$$
c_{\text {top }}\left(\Lambda^{3}(E)\right)=s_{3,1}+s_{2,2}+s_{2,1,1}+s_{1,1,1,1} .
$$

If $\operatorname{rank} E=5$ then

$$
\begin{aligned}
c_{\text {top }}\left(\Lambda^{3}(E)\right) & =9 s_{3,3,2,1,1}+3 s_{3,3,2,2}+2 s_{3,3,3,1}+9 s_{4,2,2,1,1}+3 s_{4,2,2,2}+6 s_{4,3,1,1,1} \\
& +9 s_{4,3,2,1}+3 s_{4,3,3}+3 s_{4,4,1,1}+3 s_{4,4,2}+4 s_{5,2,1,1,1}+4 s_{5,2,2,1}+4 s_{5,3,1,1} \\
& +4 s_{5,3,2}+2 s_{5,4,1}+s_{6,2,1,1}+s_{6,2,2}+s_{6,3,1}+6 s_{3,2,2,2,1}+s_{3,3,3,1}+s_{6,1,1,1,1} .
\end{aligned}
$$

If $\operatorname{rank} E=2$ then

$$
c_{n+1}\left(S^{n} E\right)=\prod_{j=0}^{(n-1) / 2}\left[j(n-j) s_{2}+\left(n^{2}-3 j(n-j)\right) s_{1,1}\right]
$$

for $n$ odd, and

$$
c_{n+1}\left(S^{n} E\right)=(n / 2) s_{1} \cdot \prod_{j=0}^{n / 2-1}\left[j(n-j) s_{2}+\left(n^{2}-3 j(n-j)\right) s_{1,1}\right]
$$

for $n$ even.

The rest of this section summarizes some results from [La-La-T] and [P3].

Let $E, F$ be vector bundles of ranks $n$ and $m$ respectively. Assume $m \geqslant n$. We state

THEOREM 2.4.

(i) [L-S1], [La-La-T] The total Segre class of the tensor product $E \otimes F$ is given by

$$
s(E \otimes F)=\sum D_{I, J}^{n, m} s_{I}(E) s_{J}(F),
$$

where the sum is over partitions $I, J$ of length $\leqslant n$ and

$$
D_{I, J}^{m, n}=\operatorname{Det}\left[\left(\begin{array}{c}
i_{p}+j_{q}+m+n-p-q \\
i_{p}+n-p
\end{array}\right)\right]_{1 \leqslant p, q \leqslant n} .
$$

(ii) [La-La-T] \& [P3] The total Segre class of the second symmetric power $S^{2} E$ is given by

$$
s\left(S^{2} E\right)=\sum\left(\left(I+\rho_{n-1}\right)\right) s_{I}(E),
$$

where the sum is over all partitions $I$ and the definition of $((J))$, for $J=\left(j_{1}>\right.$ $\left.\ldots>j_{n} \geqslant 0\right)$, is as follows. If $n$ is even, define $((J))$ to be the Pfaffian of the $n \times n$ antisymmetric matrix $\left[a_{p, q}\right]$ where for $p<q$,

$$
\left.a_{p, q}=\sum\left(\begin{array}{c}
j_{p}+j_{q} \\
j
\end{array}\right) \quad \text { (the sum over } j_{q}<j \leqslant j_{p}\right),
$$

and if $n$ is odd, then $((J)):=\sum(-1)^{p-1} 2^{j_{p}}\left(\left(J \backslash\left\{j_{p}\right\}\right)\right)$.

(iii) $[\mathrm{La}-\mathrm{La}-\mathrm{T}] \&[\mathrm{P} 3]$ The total Segre class of the second exterior power $\Lambda^{2}(E)$ is given by

$$
s\left(\Lambda^{2}(E)\right)=\sum\left[I+\rho_{n-1}\right] s_{I}(E)
$$


where the sum is over all partitions $I$ and the definition of $[J]$, for $J=\left(j_{1}>\right.$ $\left.\ldots>j_{n} \geqslant 0\right)$ is as follows. If $n$ is even, define $[J]$ to be the Pfaffian of the $n \times n$ antisymmetric matrix $\left[\left(j_{p}+j_{q}-1\right) !\left(j_{p}-j_{q}\right) / j_{p} ! j_{q} !\right] ;$ if $n$ is odd then $[J]=0$ unless $j_{n}=0$ where $[J]=\left[j_{1}, \ldots, j_{n-1}\right]$.

Remark 2.5. (Background) The history of the above formulas for $s\left(S^{2} E\right)$ and $s\left(\Lambda^{2}(E)\right)$ is as follows. At first, one of the authors of [La-La-T] has informed the author about recursive formulas for $((J))$ and $[J]$, in the form of linear equations, obtained with the help of divided differences. (We will explain and use this extremely powerful technique in Sections 3, 4 and 6.) Using this recursion the author has found and proved the above Pfaffian formulas in [P3]. Finally, the authors of [La-La-T] managed to give a self-contained and elegant account of different formulas for $s\left(S^{2} E\right)$ and $s\left(\Lambda^{2}(E)\right)$ based on an interplay between the recursive formulas, Pfaffian expressions from [P3] and formulas which present $((J))$ and $[J]$ as sums of minors in some matrices of binomial numbers. Consequently, there are no divided differences in the final version of [La-La-T]. ("The power was eliminated by the elegance"! $\left({ }^{9}\right)$ )

As it was mentioned in Section 1, the coefficients $D_{I, J}^{m, n}$ appearing in Theorem 2.4(i) are needed for the expression of the Chern-Schwartz-MacPherson classes of $D_{r}(\varphi)$ associated with an $r$-general morphism.

The analogue of Theorem 1.9 for homomorphisms with symmetries is not known yet; let us state, however, a weaker result using, this time, the numbers $((J))$ and $[J]$ from Theorem 2.4(ii) and (iii). By $\iota$ we understand the inclusion $D_{r}(\varphi) \rightarrow X$.

TheOREM 2.6 [P3]. Assume that a (possibly singular) complex analytic variety $X$ is compact, $\varphi$ is r-general and $D_{r-1}(\varphi)=\emptyset$.

(i) If $\varphi$ is symmetric then

$$
\iota_{*}\left(c_{*}\left(D_{r}(\varphi)\right)\right)=\sum(-1)^{|I|}\left(\left(I+\rho_{n-r-1}\right)\right) Q_{\rho_{n-r}+I}(E) \cap c_{*}(X),
$$

the sum over all partitions of $I$ of length $\leqslant n-r$.

(ii) If $\varphi$ is antisymmetric, $r$ even, then

$$
\iota_{*}\left(c_{*}\left(D_{r}(\varphi)\right)\right)=\sum(-1)^{|I|}\left[I+\rho_{n-r-1}\right] P_{\rho_{n-r-1}+I}(E) \cap c_{*}(X),
$$

the sum over all partitions of $I$ of length $\leqslant n-r$.

Taking the degree of the expression on the right-hand side gives the topological EulerPoincaré characteristic of $D_{r}(\varphi)$. It would be valuable to extend the theorem to $r$-general morphisms without the assumption of the emptiness of $D_{r-1}(\varphi)$.

Another application of Theorem 2.4 was given in [La-La-T] to the enumerative properties of complete correlations and quadrics. Let us limit ourselves to the latter case. Here we assume that the ground field is of characteristic different from 2 .

Let us fix a positive integer $r$ and a projective space $\mathbb{P}$. By a complete quadric of rank $r$ we understand a sequence $Q_{\bullet}: Q_{1} \subset Q_{2} \subset \cdots \subset Q_{n}$ ( $n$ can vary) of quadrics in $\mathbb{P}$, such that

1) $Q_{1}$ is nonsingular,

2) the linear span $L\left(Q_{i}\right)$ of $Q_{i}$ is the vertex of $Q_{i+1}, i=1, \ldots, n-1$,

$\left({ }^{9}\right)$ The proof of the linear equations for $((J))$ and $[J]$ via the divided differences is reproduced in Appendix A.3. 
3) $\operatorname{dim} L\left(Q_{n}\right)=r-1$.

There exists a natural structure of a nonsingular algebraic projective variety on $C Q(r)$

- the set of all rank $r$ complete quadrics (see [La-La-T] and the references therein). Let $\mu_{i} \in A_{*}(C Q(r))(i=1, \ldots, r)$ be the class of the locus of all complete quadrics $Q$ • such that $Q_{n}$ is tangent to a given (codimension $i$ )-plane in $\mathbb{P}$.

Now let $\mathcal{G}=G_{r}(\mathbb{P})$ be the Grassmannian parametrizing $(r-1)$-dimensional linear subspaces of $\mathbb{P}$. Fix a sequence $I=\left(1 \leqslant i_{1}<i_{2}<\cdots<i_{r} \leqslant \operatorname{dim} \mathbb{P}\right)$ of integers and consider the flag $L_{\bullet}: L_{1} \subset L_{2} \subset \cdots \subset L_{r}$ of linear subspaces in $\mathbb{P}$ where $\operatorname{dim} L_{j}=i_{j}$, $j=1, \ldots, r$. Let $\Omega(I)$ be the class in $A_{*}(\mathcal{G})$ of the Schubert cycle

$$
\left\{L \in \mathcal{G} \mid \operatorname{dim}\left(L \cap L_{j}\right) \geqslant j-1, j=1, \ldots, r\right\} .
$$

We have a map $f: C Q(r) \rightarrow \mathcal{G}$ such that $f\left(Q_{\bullet}\right)=L\left(Q_{n}\right)$. Let $\omega(I):=f^{*} \Omega(I)$.

Classics of enumerative geometry like Schubert, Giambelli ... were interested in the computation of the number of complete quadrics $Q_{\bullet}$ such that $Q_{n}$ is tangent to $m_{j}$ fixed planes of codimension $j$ in general position in $\mathbb{P}$ and such that $\operatorname{dim}\left(L\left(Q_{n}\right) \cap L_{j}\right) \geqslant j-1$ for each member of the above flag $L_{\bullet}$. This question makes sense if $i_{1}+\cdots+i_{r}+r-1=$ $m_{1}+\cdots+m_{r}$ because then $\mu_{1}^{m_{1}} \mu_{2}^{m_{2}} \ldots \mu_{r}^{m_{r}} \cdot \omega(I)$ is in $A_{0}(C Q(r))$. The answer to the question (under the above assumption) needs besides the numbers $((J))$ defined at the beginning of this section, also the function $\alpha(p ; k, j)$ defined by

$$
\alpha(p ; k, j):=\left\{\begin{array}{l}
\left(\begin{array}{l}
k \\
0
\end{array}\right)+\left(\begin{array}{l}
k \\
1
\end{array}\right) p+\cdots+\left(\begin{array}{l}
k \\
j
\end{array}\right) p^{j} \quad \text { if } j \geqslant 0, \\
0 \quad \text { - otherwise. }
\end{array}\right.
$$

In fact, the following result answers a more general question:

Theorem 2.7 [La-La-T]. Assume that $p$ is a number such that $0 \leqslant p<r$ and $m_{1}+$ $\cdots+m_{q}>i_{r}+i_{r-1}+\cdots+i_{r-q+1}+q-1$ for $q=1, \ldots, p-1$. Then

$$
\begin{aligned}
& \mu_{1}^{m_{1}} \mu_{2}^{m_{2}} \ldots \mu_{p+1}^{m_{p+1}} \cdot \omega(I)= \\
= & 1^{m_{1}} 2^{m_{2}} \ldots p^{m_{p}}\left[(p+1)^{m_{p+1}}((I))-\sum \alpha\left(p ; m_{p+1}, m_{p+1}-|J|-(r-p)\right) \varepsilon_{J}((J))\left(\left(J^{\prime}\right)\right)\right],
\end{aligned}
$$

where the sum is over all subsequences $J$ in $I$ of cardinality $r-p ; J^{\prime}=I \backslash J$ and $\varepsilon_{J}=\operatorname{sign}\left(J, J^{\prime}\right)$.

This theorem generalizes and offers a "modern treatment" of the results of Schubert [S] and Giambelli [G2] from the end of the previous and the beginning of the present century. For more on this subject, consult also the paper [Th] by A. Thorup in the present volume.

There is a similar formula for complete correlations which, in turn, uses the numbers $D_{I, J}^{n, m}($ see $[\mathrm{La}-\mathrm{La}-\mathrm{T}])$.

3. Flag degeneracy loci and divided differences. This section summarizes mainly some of the results of [F2]. Let

$$
F_{\bullet}: F_{1} \subset F_{2} \subset \cdots \subset F_{m}=F \quad \text { and } \quad E^{\bullet}: E=E^{n} \rightarrow \cdots \rightarrow E^{2} \rightarrow E^{1}
$$

be two flags of vector bundles over a variety $X$ and let $\varphi: F \rightarrow E$ be a vector bundle homomorphism. Assume that a function $\mathbf{r}:\{1, \ldots, n\} \times\{1, \ldots, m\} \rightarrow \mathbb{N}$ is given (we will refer to $\mathbf{r}$ as to a rank function). Define

$$
D_{\mathbf{r}}(\varphi)=\left\{x \in X \mid \operatorname{rank}\left(F_{q}(x) \rightarrow E^{p}(x)\right) \leqslant \mathbf{r}(p, q) \forall p, q\right\} .
$$


In [F2] the author gives conditions on $\mathbf{r}$ which guarantee that for a "generic" $\varphi, D_{\mathbf{r}}(\varphi)$ is irreducible. Then, a natural problem arises, to find for such an $\mathbf{r}$ and $\varphi$ a formula expressing $\left[D_{\mathbf{r}}(\varphi)\right]$ in terms of the Chern classes of $E^{\bullet}$ and $F_{\bullet}$.

It turns out that the crucial case is the case of complete flags, i.e. $\operatorname{rank} E^{i}=\operatorname{rank} F_{i}=i$ and $m=n$. The desired formula in all other cases can be deduced from that one. In this situation, the degeneracy loci $D_{\mathbf{r}}(\varphi)$ are parametrized by permutations $\mu \in S_{n}$, and

$$
\mathbf{r}_{\mu}(p, q)=\operatorname{card}\{i \leqslant p \mid \mu(i) \leqslant q\} .
$$

Let $\Omega_{\mu}\left(E^{\bullet}, F_{\bullet}\right)=D_{\mathbf{r}_{\mu}}(\varphi)$. Then the expected (i.e. the maximal one, if the locus is nonempty) codimension of $\Omega_{\mu}\left(E^{\bullet}, F_{\bullet}\right)$ is $l(\mu)$ (the length of $\mu$ ). In order to describe a formula for the fundamental class of $\Omega_{\mu}\left(E^{\bullet}, F_{\bullet}\right)$ associated with a generic $\varphi$ we need some algebraic tools developed in [B-G-G], [D1,2] and [L-S 2,3] (for an elegant account of this theory, see [M2]).

Let $A=\left(a_{1}, \ldots, a_{n}\right), B=\left(b_{1}, \ldots, b_{n}\right)$ be two sequences of independent and commuting variables. We have divided differences

defined by

$$
\partial_{i}: \mathbb{Z}[A B] \rightarrow \mathbb{Z}[A B] \quad(\text { of degree }-1)
$$

$$
\partial_{i}(f)=\left(f-\tau_{i} f\right) /\left(a_{i}-a_{i+1}\right) \quad i=1, \ldots, n-1,
$$

where $\tau_{i}=(1, \ldots, i-1, i+1, i, i+2, \ldots, n)$ denotes the $i$-th simple transposition. For every reduced decomposition $\mu=\tau_{i_{1}} \cdot \ldots \cdot \tau_{i_{k}}\left({ }^{10}\right)$ one defines $\partial_{\mu}:=\partial_{i_{1}} \circ \cdots \circ \partial_{i_{k}}$ - an operator on $\mathbb{Z}[A B]$ of degree $-l(\mu)$. In fact $\partial_{\mu}$ does not depend on the reduced decomposition chosen. Finally, for a permutation $\mu \in S_{n}$, we give, following [L5] (see also [M2]):

Definition 3.1. (Double) Schubert polynomials of Lascoux and Schützenberger. We set

$$
\mathfrak{S}_{\mu}(A / B)=\partial_{\mu^{-1} \omega} \prod_{i+j \leqslant n}\left(a_{i}-b_{j}\right)
$$

where $\omega$ is the permutation of biggest length in $S_{n}$.

Equivalently, the polynomials $\mathfrak{S}_{\mu}(A / B)$ are defined inductively by the equation

$$
\partial_{i}\left(\mathfrak{S}_{\mu}(A / B)\right)=\mathfrak{S}_{\mu \tau_{i}}(A / B)
$$

if $\mu(i)>\mu(i+1)$, the top polynomial $\mathfrak{S}_{\omega}(A / B)$ being $\prod_{i+j \leqslant n}\left(a_{i}-b_{j}\right)$.

Note that the operators act here on the $A$-variables; however, it can be shown ([L5], [M2]) that

Specialize now

$$
\mathfrak{S}_{\mu}(A / B)=(-1)^{l(\mu)} \mathfrak{S}_{\mu^{-1}}(B / A) .
$$

$$
a_{i}:=c_{1}\left(\operatorname{Ker}\left(E^{i} \rightarrow E^{i-1}\right)\right) \quad \text { and } \quad b_{i}:=c_{1}\left(F_{i} / F_{i-1}\right) .
$$

Then we have

Theorem 3.2 [F2]. Assume that $X$ is a pure-dimensional Cohen-Macaulay scheme and $\Omega_{\mu}\left(E^{\bullet}, F_{\bullet}\right)$ is of pure codimension $l(\mu)$ in $X$ or empty. Then the following equality

$\left({ }^{10}\right)$ This - most common - notation means that $\mu=(\mu(1), \ldots, \mu(n)) \in S_{n}$ is obtained from $(1, \ldots, n)$ by the sequence of simple transpositions of components, where one performs first $\tau_{i_{1}}$, then $\tau_{i_{2}}$ etc. 
holds in $A_{*}(X)$,

$$
\left[\Omega_{\mu}\left(E^{\bullet}, F_{\bullet}\right)\right]=\mathfrak{S}_{\mu}(A / B) \cap[X]
$$

The key point of the proof of Theorem 3.2 in [F2] is a geometric interpretation of the divided differences with the help of some correspondences in flag bundles. More precisely, assume, for simplicity, that $E \rightarrow X$ is a vector bundle over a nonsingular variety $X$ and let $\mathcal{F} \rightarrow X$ be the flag bundle parametrizing the flags of quotients of $E$ of successive ranks $n, n-1, \ldots, 2,1$. Denote by

$$
E^{\bullet}: E=E^{n} \rightarrow E^{n-1} \rightarrow \cdots \rightarrow E^{2} \rightarrow E^{1}
$$

the tautological flag on $\mathcal{F}$. It is well known that for $a_{i}=c_{1}\left(\operatorname{Ker}\left(E^{i} \rightarrow E^{i-1}\right)\right), i=$ $1, \ldots, n, A^{*}(\mathcal{F})$ is a quotient ring of $A^{*}(X)\left[a_{1}, \ldots, a_{n}\right]$. Let $\mathcal{F}(i)$ be the flag bundle parametrizing flags of successive quotients of ranks $n, n-1, \ldots, i+1, i-1, \ldots, 2,1$ of $E$. There is a canonical projection $\mathcal{F} \rightarrow \mathcal{F}(i)$ which is a $\mathbb{P}^{1}$-bundle. Consider the fibre product

$$
\mathcal{F} \times_{\mathcal{F}(i)} \mathcal{F}
$$

equipped with two projections

$$
p_{1}, p_{2}: \mathcal{F} \times \mathcal{F}(i) \mathcal{F} \rightarrow \mathcal{F}
$$

Proposition 3.3 [F2].

(i) The map $\left(p_{1}\right)_{*} \circ p_{2}^{*}: A^{k}(\mathcal{F}) \rightarrow A^{k+1}(\mathcal{F})$ acts on polynomials in $a_{1}, \ldots, a_{n}$ like the divided-differences operator $\partial_{i}$ does.

(ii) Assume that a flag of subbundles

$$
F_{\bullet}: F_{1} \subset F_{2} \subset \cdots \subset F_{n-1} \subset F_{n}=E
$$

is given on $X$. Then, in $A^{*}(\mathcal{F})$,

$$
\left(p_{1}\right)_{*} \circ p_{2}^{*}\left[\Omega_{\mu}\left(E^{\bullet},\left(F_{\bullet}\right)_{\mathcal{F}}\right)\right]=\left[\Omega_{\mu \tau_{i}}\left(E^{\bullet},\left(F_{\bullet}\right)_{\mathcal{F}}\right)\right]
$$

if $\mu(i)>\mu(i+1)$, and 0 - otherwise.

The theorem generalizes in a uniform way the formulas for the fundamental classes of Schubert varieties in the flag varieties from [B-G-G] and [D2], and - with the help of a rich algebra of Schubert polynomials (see [M2]) - some other known before formulas for flag degeneracy loci like the Giambelli-Thom-Porteous formula (see Section 1) as well as determinantal formulas for flag degeneracy loci from [K-L], [L1] and [P3] which we recall in the following examples. (Note that another approach to the Giambelli-Thom-Porteous formula, this time using a certain Schur complex, is given in Appendix A.6.)

ExAmple $3.4[\mathrm{~K}-\mathrm{L}]$. Assume that on $X$ a flag of vector bundles

$$
B_{1} \varsubsetneqq B_{2} \varsubsetneqq \cdots \varsubsetneqq B_{k}=B
$$

is given, with rank $B_{i}=m_{i}$. Moreover, let $\varphi: A \rightarrow B$ be a vector bundle homomorphism where $\operatorname{rank} A=n$. Consider the locus:

$$
\Omega=\left\{x \in X \mid \operatorname{dim} \operatorname{Ker}\left(B_{i}(x) \hookrightarrow B(x) \stackrel{\varphi(x)}{\longrightarrow} A(x)\right) \geqslant i, i=1, \ldots, k\right\} .
$$

Then, assuming that $X$ is a pure dimensional Cohen-Macaulay variety and $\Omega$ is of pure codimension $\sum_{i}\left(n-m_{i}+i\right)$ in $X$ or empty, one has the equality

$$
[\Omega]=\operatorname{Det}\left[c_{n-m_{i}+j}\left(A-B_{i}\right)\right]_{1 \leqslant i, j \leqslant k} \cap[X]
$$


(compare also [L1]). The author of [F2] reports on p. 417 that his approach does not cover all the instances of this formula but only the cases of the form $n-m_{1}+1>n-m_{2}+2>$ $\ldots>n-m_{k}+k$. (A similar remark applies to the formula treated in the next example.)

EXAmple $3.5[\mathrm{P} 3,(8.3)]$. Assume that on $X$ two flags of vector bundles are given

$$
B_{1} \subset B_{2} \subset \cdots \subset B_{k}=B, \quad A=A_{1} \rightarrow A_{2} \rightarrow \cdots \rightarrow A_{k-1} \rightarrow A_{k}
$$

with $\operatorname{rank} A_{i}=n_{i}$, rank $B_{i}=m_{i}$. Moreover, let $\varphi: B \rightarrow A$ be a vector bundle homomorphism. Consider the locus

$$
\Omega=\left\{x \in X \mid \operatorname{dim} \operatorname{Ker}\left(B_{i}(x) \hookrightarrow B(x) \stackrel{\varphi(x)}{\longrightarrow} A(x) \rightarrow A_{i}(x)\right) \geqslant i, i=1, \ldots, k\right\} .
$$

Then, assuming that $X$ is a pure-dimensional Cohen-Macaulay variety, $m_{i} \geqslant i$,

$$
n_{1}-m_{1}+1 \geqslant n_{2}-m_{2}+2 \geqslant \cdots \geqslant n_{k}-m_{k}+k>0
$$

and $\Omega$ is of pure codimension $\sum\left(n_{i}-m_{i}+i\right)$ in $X$ or empty, one has the equality

$$
[\Omega]=\operatorname{Det}\left[c_{n_{i}-m_{i}+j}\left(A_{i}-B_{i}\right)\right]_{1 \leqslant i, j \leqslant k} \cap[X] .
$$

See Appendix A.4 for a proof of this formula with the use of Gysin maps.

A combination of Theorem 3.2 with [G5] gives some interesting formulas for specializations of indeterminates in Schubert polynomials (see [F2, p.419]; compare also some related computations in $[\mathrm{He}-\mathrm{T}]$ using the Gröbner bases technique).

Finally, note that Schubert polynomials are a useful tool in the computation of Chern classes of the tangent vector bundles to the flag varieties — for details see [L5]. For one more application of Schubert polynomials, this time to the cohomology rings of Schubert varieties, see $[\mathrm{A}-\mathrm{L}-\mathrm{P}]$.

4. Gysin maps and divided differences. As it was pointed out in [F2], the divided differences $\partial_{i}$ are geometrically constructed from correspondences which are $\mathbb{P}^{1}$-bundles (see also the preceding section).

The aim of this section is to emphasize that some compositions of the $\partial_{i}$ 's can be interpreted geometrically as Gysin maps for flag bundles. Similar results are true for divided differences associated with other semisimple algebraic groups. We also state some "orthogonality" results with respect to Gysin maps for flag bundles.

Let $\pi: G^{1}(E) \rightarrow X$ be the projective bundle parametrizing 1-quotients of $E$ where $E$ is a vector bundle on a variety $X$ of rank $n$. Assume, for simplicity, that $X$ is smooth. Let $A=\left(a_{1}, \ldots, a_{n}\right)$ be a sequence of independent indeterminates and $\alpha_{1}, \ldots, \alpha_{n}$ - the sequence of Chern roots of $E$. One has the divided-differences operators $\partial_{i}: \mathbb{Z}[A] \rightarrow \mathbb{Z}[A]$ $(i=1, \ldots, n-1)$, associated with the simple transpositions, defined by the formulas from the preceding section.

Denote by $\triangle: \mathbb{Z}[A] \rightarrow \mathbb{Z}[A]$ the following composition of divided-differences operators

$$
\triangle:=\partial_{n-1} \circ \cdots \circ \partial_{2} \circ \partial_{1}
$$

We emphasize that while the divided-differences operators act on the whole polynomial ring $\mathbb{Z}[A]$, the symmetrizing operators appearing below are, in general, defined on proper subrings of $\mathbb{Z}[A]$ (for more on symmetrizing operators, see [L-S4] and [P4]). 
Proposition 4.1. One has, for $P \in \mathbb{Z}[A]^{S_{1} \times S_{n-1}}$,

$$
\begin{gathered}
\triangle P=\sum_{\bar{\sigma} \in S_{n} / S_{1} \times S_{n-1}} \sigma\left(\frac{P}{\prod_{\beta \geqslant 2}\left(a_{1}-a_{\beta}\right)}\right) \\
\pi_{*}\left(P\left(\alpha_{1}, \ldots, \alpha_{n}\right)\right)=(\Delta P)\left(\alpha_{1}, \ldots, \alpha_{n}\right) .
\end{gathered}
$$

A word about how one proves such a result (this method can also be applied to other results of this type stated in this section). The equality (i) is just a straightforward verification. In fact, since $\Delta(\sigma P)=\sigma \Delta(P)$ for $\sigma \in S_{n}$, it suffices to check it for $P=a_{1}^{k}, k=0,1, \ldots, n-1$. For the degree reasons it remains to show (i) for $P=a_{1}^{n-1}$, the calculation of the expression on the right-hand side being essentially the Laplace development of the Vandermonde determinant. To show (ii) we can assume without loss of generality that $X$ is a "big" Grassmannian and $E$ is a universal bundle on it with the Chern roots $a_{1}, \ldots, a_{n}$. Then $\Delta$ induces an $A^{*}(X)$-morphism. Since $A^{*}\left(G^{1} E\right)=$ $\bigoplus_{i=1}^{n-1} \pi^{*} A^{*}(X) \xi^{i}$, where $\xi=c_{1}(\mathcal{O}(1))$, the assertion follows from the equality $\triangle\left(a_{1}^{n-1}\right)=$ 1 because $\pi_{*}\left(\xi^{n-1}\right)=1$ and $\pi_{*}\left(\xi^{i}\right)=0$ for $i<n-1$.

Let $\pi: G^{q}(E) \rightarrow X$ be the Grassmannian bundle parametrizing $q$-quotients of the bundle $E$ as above. Denote by $\square: \mathbb{Z}[A] \rightarrow \mathbb{Z}[A]$ the following composition of divideddifferences operators $(r=n-q)$ :

$$
\square:=\left(\partial_{r} \circ \cdots \circ \partial_{2} \circ \partial_{1}\right) \circ \cdots \circ\left(\partial_{n-2} \circ \cdots \circ \partial_{q} \circ \partial_{q-1}\right) \circ\left(\partial_{n-1} \circ \cdots \circ \partial_{q+1} \circ \partial_{q}\right) .
$$

Proposition 4.2. One has, for $P \in \mathbb{Z}[A]^{S_{q} \times S_{r}}$,

$$
\begin{aligned}
& \square P=\sum_{\bar{\sigma} \in S_{n} / S_{q} \times S_{r}} \sigma\left(\frac{P}{\prod_{\alpha \leqslant q<\beta}\left(a_{\alpha}-a_{\beta}\right)}\right) \\
& \pi_{*}\left(P\left(\alpha_{1}, \ldots, \alpha_{n}\right)\right)=(\square P)\left(\alpha_{1}, \ldots, \alpha_{n}\right) .
\end{aligned}
$$

The operator $\square$ is known in interpolation theory as the Lagrange-Sylvester symmetrizer. We refer the reader to [L4] for an account of algebraic properties of the LagrangeSylvester symmetrizer.

Let now $\tau=\tau_{E}: \operatorname{Fl}(E)=\mathrm{Fl}^{n, n-1, \ldots, 1}(E) \rightarrow X$ be the flag bundle endowed with the tautological sequence of quotients

$$
E=Q^{n} \rightarrow Q^{n-1} \rightarrow \cdots \rightarrow Q^{2} \rightarrow Q^{1},
$$

where $\operatorname{rank} Q^{i}=i$. Let $L_{i}=\operatorname{Ker}\left(Q^{i} \rightarrow Q^{i-1}\right)$ and $\alpha_{i}=c_{1}\left(L_{i}\right), i=1, \ldots, n$.

Denote by $\partial=\partial_{A}: \mathbb{Z}[A] \rightarrow \mathbb{Z}[A]$ the following composition of divided differences:

$$
\partial:=\left(\partial_{1} \circ \cdots \circ \partial_{n-1}\right) \circ \cdots \circ\left(\partial_{1} \circ \partial_{2} \circ \partial_{3}\right) \circ\left(\partial_{1} \circ \partial_{2}\right) \circ \partial_{1}
$$

In other words, $\partial=\partial_{\omega}$ is the operator associated with the longest element $\omega \in S_{n}$ (in the notation of Section 3).

Proposition 4.3. One has, for $P \in \mathbb{Z}[A]$,

$$
\partial P=\sum_{\sigma \in S_{n}} \sigma\left(\frac{P}{\prod_{\alpha<\beta}\left(a_{\alpha}-a_{\beta}\right)}\right)
$$




$$
\tau_{*}\left(P\left(\alpha_{1}, \ldots, \alpha_{n}\right)\right)=(\partial P)\left(\alpha_{1}, \ldots, \alpha_{n}\right) .
$$

Let us prove (ii). Consider the factorization

$$
\tau=\tau_{E}: \operatorname{Fl}(E) \cong \operatorname{Fl}\left(Q^{n-1}\right) \stackrel{\tau^{\prime}=\tau_{Q^{n-1}}}{\longrightarrow} G_{1} E \stackrel{\pi}{\longrightarrow} X .
$$

Hence $\tau_{*}=\pi_{*} \circ \tau_{*}^{\prime}$. Define the operator $\partial^{\prime}: \mathbb{Z}[A] \rightarrow \mathbb{Z}[A]$ by

$$
\partial^{\prime}=\left(\partial_{1} \circ \cdots \circ \partial_{n-2}\right) \circ \cdots \circ\left(\partial_{1} \circ \partial_{2}\right) \circ \partial_{1} .
$$

Thus $\partial=\Delta \circ \partial^{\prime}$. Then assuming that equation (ii) is valid for $\tau^{\prime}$ and $\partial^{\prime}$ instead of $\tau$ and $\partial$, and invoking Proposition 4.1 we deduce, by induction on $n$, that equation (ii) holds.

The operator $\partial$ is called the Jacobi symmetrizer. A familiar Jacobi-Trudi identity can be restated as

Proposition 4.4. Let $I$ be a partition, $l(I) \leqslant n$. Then

$$
\partial\left(a_{1}^{i_{1}+n-1} a_{2}^{i_{2}+n-2} \ldots a_{n-1}^{i_{n-1}+1} a_{n}^{i_{n}}\right)=s_{I}(A) .
$$

For a simple operator proof see Appendix A.5. In fact this identity is valid for sequences $I \in \mathbb{Z}^{n}$ such that $i_{1} \geqslant-(n-1), \ldots, i_{n-1} \geqslant-1, i_{n} \geqslant 0$ (notation: $I \geqslant-\rho_{n-1}$ ).

Using this proposition one can give a short operator proof of Proposition 1.3(i). Denoting $A_{q}=\left(a_{1}, \ldots, a_{q}\right)$ and $A^{r}=\left(a_{q+1}, \ldots, a_{n}\right)$ and assuming $\mathbb{Z}^{q} \ni I \geqslant-\rho_{q-1}$, $\mathbb{Z}^{r} \ni J \geqslant-\rho_{r-1}$, we obtain

$$
\begin{aligned}
\square\left(s_{I}\left(A_{q}\right) \cdot s_{J}\left(A^{r}\right)\right) & =\square\left(\partial_{A_{q}}\left(a_{1}^{i_{1}+q-1} \cdot \ldots \cdot a_{q}^{i_{q}}\right) \cdot \partial_{A^{r}}\left(a_{q+1}^{j_{1}+r-1} \cdot \ldots \cdot a_{n}^{j_{r}}\right)\right) \\
& =\partial_{A}\left(a_{1}^{i_{1}+q-1} \cdot \ldots \cdot a_{q}^{i_{q}} a_{q+1}^{j_{1}+r-1} \cdot \ldots \cdot a_{n}^{j_{r}}\right) \\
& =\partial_{A}\left(a_{1}^{\left(i_{1}-r\right)+(n-1)} \cdot \ldots \cdot a_{q}^{\left(i_{q}-r\right)+(n-q)} a_{q+1}^{j_{1}+r-1} \cdot \ldots \cdot a_{n}^{j_{r}}\right) \\
& =s_{i_{1}-r, \ldots, i_{q}-r, j_{1}, \ldots, j_{r}}(A),
\end{aligned}
$$

which is the algebraic essence of the proposition (the second equality being a straightforward verification).

Let us now pass to other root systems than the one of type $A_{n-1}$. We need more notation which will also be used in Section 6 .

Let $G$ be a semisimple algebraic group, $B \subset G-$ a Borel subgroup and $T \subset B$ - a maximal torus. With a character $\chi \in X(T):=\operatorname{Hom}\left(T, \mathbb{C}^{*}\right)$ one associates a line bundle $L_{\chi}$ over a generalized flag variety $G / B$. The total space of $L_{\chi}$ is $G \times \mathbb{C} / \sim$, where the relation " " is defined by: $(g, z) \sim\left(g b, \chi\left(b^{-1}\right) z\right)$ for $g \in G, z \in \mathbb{C}, b \in B$; recall that $X(T)=\operatorname{Hom}\left(B, \mathbb{C}^{*}\right)$. We have a map from $X(T)$ to $A^{1}(G / B)$ which assigns to $\chi$ the Chern class $c_{1}\left(L_{\chi}\right)$. It extends multiplicatively to the Borel characteristic map:

$$
c: S^{\bullet}(X(T)) \rightarrow A^{*}(G / B) .
$$

Let $R$ be the root system of $(G, T)$, endowed with the basis $\triangle$ associated with $B$, and $P \supset B$ be the parabolic subgroup associated with a subset $\theta \subset \triangle$. Denote by $W_{\theta}$ the subgroup of the Weyl group $W$ of $(G, T)$ or $R$ generated by all reflections $\left\{s_{\alpha}\right\}_{\alpha \in \theta}$. Then the characteristic map restricted to the $W_{\theta}$-invariants factorizes through $A^{*}(G / P)$ :

$$
c: S^{\bullet}(X(T))^{W_{\theta}} \rightarrow A^{*}(G / P) .
$$

Let $R_{\theta}$ (resp. $R_{\theta}^{+}$) denote the subset of the set of roots (resp. positive roots with respect to $B$ ) formed by linear combinations of roots in $\theta$ and $d_{\theta}=\prod_{\alpha \in R_{\theta}^{+}} \alpha$. Consider a 
"symmetrizing operator" $\partial^{\theta}: S^{\bullet}(X(T)) \rightarrow S^{\bullet}(X(T))$ defined by

$$
\partial^{\theta}(f)=\sum_{w \in W_{\theta}}(-1)^{l(w)} w(f) / d_{\theta},
$$

where $l(w)$ is the length of $w$ with respect to $\triangle$.

THEOREM 4.5 [A-C].

(a)

(b) The diagram

$$
\partial^{\theta}\left(S^{\bullet}(X(T))\right) \subset S^{\bullet}(X(T))^{W_{\theta}} .
$$

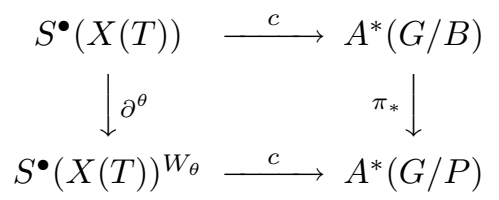

commutes, where $\pi_{*}$ is the Gysin map associated with $\pi: G / B \rightarrow G / P$. In other words, $\pi_{*}$ is induced by $\partial^{\theta}$.

Observe that for $\theta=\Delta, \partial^{\Delta}(f)=\sum(-1)^{l(w)} w f / d$ where $d$ is a product of all positive roots and the sum is over $w \in W$. This formula gives, more generally, a symmetrizing operator description of the Gysin maps associated with $G / B$-fibrations $\left({ }^{11}\right)$ overlapping, e.g., the case of Lagrangian and orthogonal flag bundles.

Let us recall another familiar interpretation of $\partial^{\Delta}$. Given a root $\alpha \in R$, one defines an operator $\partial_{\alpha}: S^{\bullet}(X(T)) \rightarrow S^{\bullet}(X(T))$ by

$$
\partial_{\alpha} f=\left(f-s_{\alpha} f\right) / \alpha,
$$

where $s_{\alpha} \in W$ is the reflection associated with $\alpha$.

Lemma 4.6 [B-G-G], [D1]. If $w \in W, l(w)=k$ and $w=s_{\alpha_{1}} \cdot \ldots \cdot s_{\alpha_{k}}=s_{\beta_{1}} \cdot \ldots \cdot s_{\beta_{k}}$ where $\alpha_{i}, \beta_{i} \in \Delta$, then

$$
\partial_{\alpha_{1}} \circ \ldots \circ \partial_{\alpha_{k}}=\partial_{\beta_{1}} \circ \ldots \circ \partial_{\beta_{k}}
$$

Thus the value of this operator can be denoted by $\partial_{w}$ without ambiguity.

It has been shown in loc.cit. that for the longest element $w_{0} \in W, \partial_{w_{0}}=\partial^{\Delta}$. Since $\theta$ is a basis of the root (sub)system $R_{\theta} \subset R$, the operator $\partial^{\theta}$ is similarly interpreted as the operator associated with the longest word in $W_{\theta}$. (Of course, the operators considered in Section 3 and in the beginning of this section are special cases of the ones here for type $A_{n-1}$, the Jacobi symmetrizer being $\left.\partial^{\Delta}=\partial_{(n, n-1, \ldots, 1)}\right)$.

Taking as the starting point the above mentioned symmetrizing operator description of the Gysin map associated with a $G / B$-fibration, the authors of [P-R5] give some explicit formulas for the Gysin maps associated with Lagrangian and orthogonal Grassmannian bundles. Consider, for example, the Lagrangian case. Let $V \rightarrow X$ be a vector bundle

$\left({ }^{11}\right)$ Note added in proof: This has circulated as a "folklore" among specialists since some time. A precise written account for $f_{*}: A_{*}(X / B) \rightarrow A_{*}(Y)$, where $f: X \rightarrow Y$ is a principal $G$-bundle ( $X$ and $Y$ are nonsingular varieties, $G$ is a reductive group), is contained in a paper by M. Brion The push-forward and Todd class of flag bundles - in this volume. In this paper, the author gives also a symmetrizing operator description of the Gysin map associated with a flag bundle $X / P \rightarrow Y$ where $P \subset G$ is a parabolic subgroup (thus generalizing the general linear group case from $[\mathrm{P} 3]$ and the results of $[\mathrm{A}-\mathrm{C}])$. Moreover, he computes the Todd class of the tangent bundle of such a "flag fibration" $X / P \rightarrow Y$. 
of rank $2 n$ endowed with a nondegenerate antisymmetric form. Let $\tau: \operatorname{LFl}(V) \rightarrow X$ be the flag bundle parametrizing flags of isotropic subbundles of ranks $1,2, \ldots, n$ in $V$ with respect to the above form, and let $\pi: L G_{n}(V) \rightarrow X$ be the Lagrangian Grassmannian bundle parametrizing top-dimensional isotropic subbundles in $V$. Let $\alpha_{1}, \ldots, \alpha_{n}$ be the sequence of the Chern roots of the tautological Lagrangian (sub)bundle $R$ on $L G_{n}(V)$. We know by the above that one has in $A^{*}(X)$,

$$
\tau_{*}\left(f\left(\alpha_{1}, \ldots, \alpha_{n}\right)\right)=\left(\partial_{(\overline{1}, \overline{2}, \ldots, \bar{n})} f\right)\left(\alpha_{1}, \ldots, \alpha_{n}\right),
$$

where $f$ is a polynomial in $n$ variables. (Recall that the symplectic Weyl group is the group of barred permutations and $(\overline{1}, \overline{2}, \ldots, \bar{n})$ is the longest element of it.)

In connection with $\pi_{*}$, it is natural to associate with a vector bundle $E$ the following polynomials in its Chern classes (consult [P-R5] and the subsection A connection with $Q$-polynomials in Section 6). We set $\widetilde{Q}_{i} E=c_{i}(E)$ and for a partition $I, l(I) \geqslant 2$, to define $\widetilde{Q}_{I} E$ we mimic the definition of the Schur polynomial $Q_{I}$ (see Section 1).

Proposition 4.7 [P-R5]. One has in $A^{*}(X)$ :

(i) $\pi_{*}\left(f\left(\alpha_{1}, \ldots, \alpha_{n}\right)\right)=\left(\partial_{(\bar{n}, \overline{n-1}, \ldots, \overline{1}, \overline{1})} f\right)\left(\alpha_{1}, \ldots, \alpha_{n}\right)$ for a symmetric polynomial $f$ in $n$ variables.

(ii) The element $\widetilde{Q}_{I} R^{\vee}$ has a nonzero image under $\pi_{*}$ only if each number $p, 1 \leqslant p \leqslant n$, appears as a part of $I$ with an odd multiplicity $m_{p}$. If the latter condition holds then

$$
\pi_{*} \widetilde{Q}_{I} R^{\vee}=\prod_{p=1}^{n}\left((-1)^{p} c_{2 p} V\right)^{\left(m_{p}-1\right) / 2} .
$$

(iii) The element $s_{I} R^{\vee}$ has a nonzero image under $\pi_{*}$ only if $I$ is the partition of the form $2 J+\rho_{n}$ for some partition $J$. If $I=2 J+\rho_{n}$ then

$$
\pi_{*} s_{I} R^{\vee}=s_{J}^{[2]} V \text {, }
$$

where the right-hand side is defined as follows: if $s_{J}=P(e$.$) is a unique presentation$ of $s_{J}$ as a polynomial in the elementary symmetric functions $e_{i}, E-a$ vector bundle, then $s_{J}^{[2]}(E):=P$ with $e_{i}$ replaced by $(-1)^{i} c_{2 i}(E)(i=1,2, \ldots)$.

We finish this section with some examples of the "orthogonality" results for Gysin maps for flag bundles. These results are crucial for computing the classes of diagonals in flag bundles and the knowledge of the classes of diagonals is useful in calculations of the classes of Schubert varieties, following a procedure described in the next section.

In the next theorem we follow the previously introduced notation from this section. Additionally, we put $\mathfrak{S}_{\mu}(A)=\mathfrak{S}_{\mu}(A / 0, \ldots, 0)\left({ }^{12}\right)$ for $\mu \in S_{n}$, in the notation of Sec-

$\left({ }^{12}\right)$ Note added in proof: It is an interesting question to extend the above definition of Schubert polynomials $\mathfrak{S}_{\mu}(A)$ to other semisimple groups. It appears that for the symplectic and orthogonal groups, a satisfactory algebro-combinatorial theory of this type has been given by S. Billey and M. Haiman in Schubert polynomials for classical groups, J. Amer. Math. Soc. 8 (1995), 443-482. Compare also: S. Fomin and A. N. Kirillov, Combinatorial Bn analogues of Schubert polynomials and S. Billey, Transition equations for isotropic flag manifolds - preprints (1995). For a theory of Schubert polynomials, suited to algebraic geometry, which has grown up from a paper by P. Pragacz and J. Ratajski [P-R5], see a forthcoming article: Symplectic and orthogonal Schubert polynomials à la polonaise - in preparation in collaboration with A. Lascoux. 
tion 3. Moreover, we define the polynomials $\widetilde{Q}_{I} A$ as follows. We set $\widetilde{Q}_{i} A=e_{i}(A)$ (the $i$-th elementary symmetric polynomial in $A$ ), and, for a strict partition $I, l(I) \geqslant 2$, we mimic the definition of the Schur $Q$-polynomial $Q_{I}$ (thus the element $\widetilde{Q}_{I} E$ associated above with a bundle $E$ is equal to $\widetilde{Q}_{I} A$ with $A$ specialized to the Chern roots of $E$ ). Note that the algebro-geometric properties of these $\widetilde{Q}$-polynomials were worked out in [P-R5] (see also [La-Le-T2] where an interesting specialization of a Hall-Littlewood polynomial, denoted in loc.cit. by $Q_{I}^{\prime}$, is studied, giving, for the specialization $q=-1$ of the parameter, the "Young dual" of $\left.\widetilde{Q}_{I}\right)$.

THEOREM 4.8 .

(i) For partitions $I, J \subset(r)^{q}$,

$$
\square\left(s_{I}\left(A_{q}\right) \cdot s_{\bar{J}}\left(-a_{r+1}, \ldots,-a_{n}\right)\right)=\delta_{I, J},
$$

where the Ferrers' diagram of $\bar{J}$ is the complement of the one of $J^{\sim}$ in $(q)^{r}$. Using a standard $\lambda$-ring notation, this is equivalently rewritten as

$$
\square\left(s_{I}\left(A_{q}\right) \cdot s_{(r)^{q} / J}\left(-A^{r}\right)\right)=\delta_{I, J} .
$$

(ii) For permutations $\mu, \nu \in S_{n}$,

$$
\partial_{\omega}\left(\mathfrak{S}_{\mu}(A) \cdot \mathfrak{S}_{\nu \omega}\left(-a_{n},-a_{n-1}, \ldots,-a_{1}\right)\right)=\delta_{\mu, \nu} .
$$

(iii) For strict partitions $I, J \subset \rho_{n}=(n, n-1, \ldots, 2,1)$, one has the following equality, in the symplectic case:

$$
\partial_{(\bar{n}, \overline{n-1}, \ldots, \overline{2}, \overline{1})}\left(\widetilde{Q}_{I}(A) \cdot \widetilde{Q}_{\rho_{n} \backslash J}(A)\right)=\delta_{I, J} .
$$

Here, $\delta_{.,}$. denotes the Kronecker delta and $\rho_{n} \backslash J$ is the strict partition whose parts complement the parts of $J$ in $\{1, \ldots, n\}$.

Assertion (i) can be deduced from (the $\square$-version of) Proposition 1.3(i). For a proof of (ii), see [L-S6], [M2] or [L-P]. Assertion (iii) stems from [P-R5].

5. Fundamental classes, diagonals and Gysin maps. As it was pointed out in Section 1, one of the fundamental problems in the study of a concrete (closed) subscheme of a given (smooth) scheme $X$ is the computation of its fundamental class in terms of given generators of the Chow ring of $X$.

The decisive role in the method described in this section is played by the diagonal of the ambient scheme or, more precisely, its class in the corresponding Chow group of a fibre product $\left({ }^{13}\right)$. As a matter of fact, we have already seen, in Section 3, one application of the diagonal to the computation of fundamental classes. In the situation of Section 3, there is a vector bundle on the product of flag bundles endowed with a section vanishing precisely on the diagonal. The top Chern class of this bundle is represented by $\mathfrak{S}_{\omega}(A / B)$. By applying divided differences to this polynomial, one gets polynomials representing the classes of other (i.e. higher dimensional) degeneracy loci in the product of flag bundles. (This generalizes the procedure from [B-G-G] and [D2]: starting from the class of the point and applying divided differences one gets the class of a curve, then the class of a surface, etc.) The procedure given below is of different nature. By using a

$\left({ }^{13}\right)$ The material of this section is due to the author. A discovery of this method is inspired by the construction used in the proof of the main formula in the paper by G. Kempf and D. Laksov [K-L]. 
desingularization of the subscheme whose class we want to compute, and the diagonal of the ambient scheme, we replace the original problem by the one of computing the image of the class of the diagonal under an appropriate Gysin map. Moreover, since the diagonal is not always represented as the scheme of zeros of a vector bundle (this seems to happen, e.g., for flag bundles for classical groups different from $S L_{n}$ ), we give a recipe allowing to calculate the class of the diagonal of the fibre product with the help of Gysin maps.

Let $S$ be a smooth scheme (over a field) and $\pi: X \rightarrow S$ a smooth morphism of schemes. Suppose that $D \subset X$ is a (closed) subscheme whose class is to be computed. Let $p: Z \rightarrow S$ be a proper smooth morphism and $\alpha: Z \rightarrow X$ be an $S$-morphism which maps birationally $Z$ onto $D$. Assume that $\alpha$ is proper. Consider a commutative diagram:

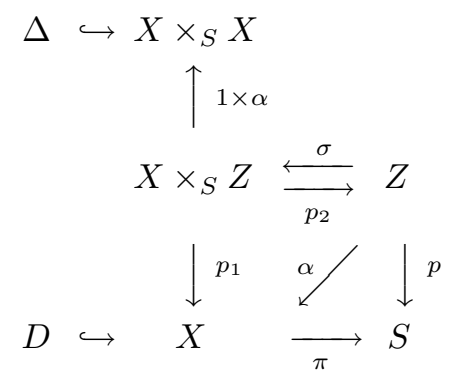

Here $p_{1}$ and $p_{2}$ are the projections, the section $\sigma$ (of $p_{2}$ ) equals $\alpha \times_{S}$ id and $\Delta$ is the diagonal in the fibre product $X \times_{S} X$.

Proposition 5.1. Suppose that the class of the diagonal $\Delta$ in $A^{*}\left(X \times_{S} X\right)$ is $[\Delta]=$ $\sum \operatorname{pr}_{1}^{*}\left(x_{i}\right) \cdot \operatorname{pr}_{2}^{*}\left(y_{i}\right)$ where $\operatorname{pr}_{i}: X \times_{S} X \rightarrow X$ are the projections and $x_{i}, y_{i} \in A^{*}(X)$. Then, in $A^{*}(X)$,

$$
[D]=\sum_{i} x_{i} \cdot\left(\pi^{*} p_{*} \alpha^{*}\left(y_{i}\right)\right) .
$$

Proof. By the assumption $[D]=\alpha_{*}([Z])$. Since $\alpha=p_{1} \circ \sigma$, we have $\alpha_{*}([Z])=$ $\left(p_{1}\right)_{*}[\sigma(Z)]$. Now, the key observation is that, in the scheme-theoretic sense, one has the equality $\sigma(Z)=(1 \times \alpha)^{-1}(\Delta)$. Since $\Delta \cong X$ is smooth, this implies $[\sigma(Z)]=(1 \times \alpha)^{*}([\Delta])$ (see Lemma 9 in $[\mathrm{K}-\mathrm{L}])$. We then have:

$$
\begin{aligned}
{[D] } & =\left(p_{1}\right)_{*}([\sigma(Z)])=\left(p_{1}\right)_{*}\left((1 \times \alpha)^{*}([\Delta])\right) \\
& =\left(p_{1}\right)_{*}\left((1 \times \alpha)^{*}\left(\sum_{i} \operatorname{pr}_{1}^{*}\left(x_{i}\right) \cdot \operatorname{pr}_{2}^{*}\left(y_{i}\right)\right)\right) \\
& =\left(p_{1}\right)_{*}\left(\sum_{i} p_{1}^{*}\left(x_{i}\right) \cdot p_{2}^{*}\left(\alpha^{*}\left(y_{i}\right)\right)\right) \\
& =\sum_{i} x_{i} \cdot\left(\left(p_{1}\right)_{*} p_{2}^{*} \alpha^{*}\left(y_{i}\right)\right) \\
& =\sum_{i} x_{i} \cdot\left(\pi^{*} p_{*} \alpha^{*}\left(y_{i}\right)\right),
\end{aligned}
$$

where the last equality follows from the above fibre product diagram and [F1, Proposition 1.7 . 
The next result shows how one can compute the fundamental class of the diagonal $[\Delta] \in A^{*}\left(X \times_{S} X\right)$ using Gysin maps.

TheOREm 5.2. Let $S$ be as above and $\pi: X \rightarrow S$ be a proper smooth morphism such that $\pi^{*}$ makes $A^{*}(X)$ a free $A^{*}(S)$-module; $A^{*}(X)=\bigoplus_{\alpha \in \Lambda} A^{*}(S) \cdot a_{\alpha}$, where $a_{\alpha} \in$ $A^{n_{\alpha}}(X)$ and $A^{*}(X)=\bigoplus_{\beta \in \Lambda} A^{*}(S) \cdot b_{\beta}$, where $b_{\beta} \in A^{m_{\beta}}(X)$. Suppose that for any $\alpha$ there is a unique $\beta=$ : $\alpha^{\prime}$ such that $n_{\alpha}+m_{\beta}=\operatorname{dim} X-\operatorname{dim} S$ and $\pi_{*}\left(a_{\alpha} \cdot b_{\beta}\right) \neq 0$ (assume $\left.\pi_{*}\left(a_{\alpha} \cdot b_{\alpha^{\prime}}\right)=1\right)$. Moreover, denoting by $\mathrm{pr}_{i}: X \times{ }_{S} X \rightarrow X(i=1,2)$ the projections, suppose that the homomorphism $A^{*}(X) \otimes_{A^{*}(S)} A^{*}(X) \rightarrow A^{*}\left(X \times{ }_{S} X\right)$, defined by $g \otimes h \mapsto \operatorname{pr}_{1}^{*}(g) \cdot \operatorname{pr}_{2}^{*}(h)$, is an isomorphism. Then

(i) The class of the diagonal $\Delta$ in $X \times{ }_{S} X$ equals $[\Delta]=\sum_{\alpha, \beta} d_{\alpha \beta} a_{\alpha} \otimes b_{\beta}$, where, for any $\alpha, \beta, \quad d_{\alpha \beta}=P_{\alpha \beta}\left(\left\{\pi_{*}\left(a_{\kappa} \cdot b_{\lambda}\right)\right\}\right)$ for some polynomial $P_{\alpha \beta} \in \mathbb{Z}\left[\left\{x_{\kappa \lambda}\right\}\right]$.

(ii) The following conditions are equivalent:

a) One has $\pi_{*}\left(a_{\alpha} \cdot b_{\beta^{\prime}}\right)=\delta_{\alpha, \beta}$, the Kronecker delta.

b) The class of the diagonal $\Delta \subset X \times_{S} X$ equals $[\Delta]=\sum_{\alpha} a_{\alpha} \otimes b_{\alpha^{\prime}}$.

Proof. Denote by $\delta: X \rightarrow X \times{ }_{S} X, \delta^{\prime}: X \rightarrow X \times X$ (× denotes the Cartesian product) the diagonal embeddings and by $\gamma$ the morphism $\pi \times{ }_{S} \pi: X \times_{S} X \rightarrow S$. For $g, h \in A^{*}(X)$ we have

$$
\pi_{*}(g \cdot h)=\pi_{*}\left(\left(\delta^{\prime}\right)^{*}(g \times h)\right)=\pi_{*}\left(\delta^{*}(g \otimes h)\right)=\gamma_{*} \delta_{*}\left(\delta^{*}(g \otimes h)\right)=\gamma_{*}([\Delta] \cdot(g \otimes h)),
$$

where all the equalities follow from the theory in [F1, Chap. 8] by taking into account, for the second one, the commutative diagram

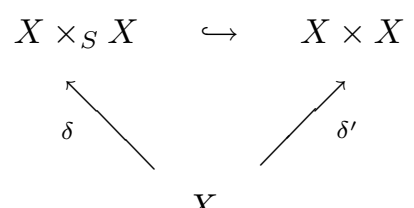

and, for the third one, the equality $\pi=\gamma \circ \delta$. Hence, writing $[\Delta]=\sum d_{\mu \nu} b_{\mu} \otimes a_{\nu}$, we get

$$
\begin{aligned}
\pi_{*}\left(a_{\alpha} \cdot b_{\beta}\right) & =\gamma_{*}\left([\Delta] \cdot\left(a_{\alpha} \otimes b_{\beta}\right)\right)=\left(\pi_{*} \otimes \pi_{*}\right)\left(\left(\sum d_{\mu \nu} b_{\mu} \otimes a_{\nu}\right) \cdot\left(a_{\alpha} \otimes b_{\beta}\right)\right) \\
& =\sum_{\mu, \nu} d_{\mu \nu} \pi_{*}\left(b_{\mu} \cdot a_{\alpha}\right) \cdot \pi_{*}\left(a_{\nu} \cdot b_{\beta}\right) .
\end{aligned}
$$

(i) By the assumption and (*) with $\alpha$ replaced by $\alpha^{\prime}$, and $\beta$ by $\beta^{\prime}$, we get

$$
d_{\alpha \beta}=\pi_{*}\left(a_{\alpha^{\prime}} \cdot b_{\beta^{\prime}}\right)-\sum_{\mu \neq \alpha, \nu \neq \beta} d_{\mu \nu} \pi_{*}\left(b_{\mu} \cdot a_{\alpha^{\prime}}\right) \cdot \pi_{*}\left(a_{\nu} \cdot b_{\beta^{\prime}}\right) .
$$

where the degree of $d_{\mu \nu} \in A^{*}(S)$ such that $\pi_{*}\left(b_{\mu} \cdot a_{\alpha^{\prime}}\right) \cdot \pi_{*}\left(a_{\nu} \cdot b_{\beta^{\prime}}\right) \neq 0$ and $\mu \neq \alpha$ or $\nu \neq \beta$, is smaller than the degree of $d_{\alpha \beta}$. The assertion now follows by induction on the degree of $d_{\alpha \beta}$.

(ii) a) $\Rightarrow$ b): By virtue of a), Equation $(* *)$ now reads $\pi_{*}\left(a_{\alpha^{\prime}} \cdot b_{\beta^{\prime}}\right)=d_{\alpha \beta}$ and immediately implies b).

b) $\Rightarrow$ a): Without loss of generality we can assume that $\Lambda$ is endowed with a linear ordering $\prec$ compatible with codimension, i.e. $n_{\alpha_{1}}<n_{\alpha_{2}} \Rightarrow \alpha_{1} \prec \alpha_{2}, m_{\beta_{1}}<m_{\beta_{2}} \Rightarrow \beta_{1} \prec \beta_{2}$ and such that $\alpha_{1} \prec \alpha_{2} \Rightarrow \alpha_{2}^{\prime} \prec \alpha_{1}^{\prime}$. The rows and columns of the matrices below are ordered 
using the ordering $\prec$. Write $x_{\alpha \beta}=\pi_{*}\left(a_{\alpha} \cdot b_{\beta}\right)$. By virtue of $\left.\mathrm{b}\right)$, Equation $(*)$ gives us the following system of equations:

$$
x_{\alpha \beta}=\sum_{\mu} x_{\alpha \mu} x_{\mu^{\prime} \beta},
$$

where $\alpha, \beta \in \Lambda$. Note that the antidiagonal of the matrix $M:=\left[x_{\alpha \beta}\right]_{\alpha, \beta \in \Lambda}$ is indexed by $\left\{\left(\alpha, \alpha^{\prime}\right) \mid \alpha \in \Lambda\right\}$. The assumption implies that this antidiagonal consists of units. Moreover, because of dimension reasons and the assumption again, we know that the entries above the diagonal are zero. Let $P$ be the permutation matrix corresponding to the bijection $\alpha \mapsto \alpha^{\prime}$ of $\Lambda$. The above system of equations is rewritten in the matrix form as:

$$
M P=M P \cdot M P .
$$

Then $M P$ as a (lower) triangular matrix with the units on the diagonal, must be the identity matrix. Hence $M=P^{-1}$ and this implies a).

Remark 5.3. A standard situation when the theorem can be applied is when $\pi$ : $X \rightarrow S$ is a locally trivial fibration and $\left\{a_{\alpha}\right\},\left\{b_{\beta}\right\}$ restrict to bases of the Chow ring of a fibre $F$ which are dual under the Poincaré duality map: $(a, b) \mapsto \int_{F} a \cdot b$. In such a situation the above method is successfully applied in [P-R5] to solve a problem of J. Harris - for more on that, see the next section.

Example 5.4. Let $\pi: \mathcal{G} \rightarrow X$ be a relative Grassmannian as in Proposition 1.3. It is easy to see the diagonal in $\mathcal{G}_{1} \times_{X} \mathcal{G}_{2}$, where $\mathcal{G}_{1}=\mathcal{G}_{2}=\mathcal{G}$, is given (in the schemetheoretic sense) by the vanishing of the entries of a matrix of the homomorphism $R_{\mathcal{G}_{1}} \rightarrow$ $E_{\mathcal{G}_{1}}=E_{\mathcal{G}_{2}} \rightarrow Q_{\mathcal{G}_{2}}$. Hence, by the theorem and a formula for the top Chern class of the tensor product [L2], we have that $\pi_{*}\left(s_{I}(Q) \cdot s \bar{J}\left(R^{\vee}\right)\right)=\delta_{I, J}$ for $I, J \subset(r)^{q}$, where $\bar{J}$ is the partition whose Ferrers' diagram complements the one of $J^{\sim}$ in the rectangle $(q)^{r}$. Equivalently, $\pi_{*}\left(s_{I}(Q) \cdot s_{(r)^{q} / J}(-R)\right)=\delta_{I, J}$. This is coherent with Theorem 4.8(i) (invoking Proposition 4.2).

In a similar way, using the calculation of the class of the diagonal from [F2, Proposition $7.5]$ via the top Chern class of a suitable vector bundle, one can reprove the equality in Theorem 4.8(ii). On the contrary, it appears that the equality in Theorem 4.8(iii) does not admit a geometric interpretation in terms of the top Chern class of a vector bundle on a fibre product of two Lagrangian Grassmannian bundles.

6. Intersection rings of spaces $G / P$, divided differences and formulas for isotropic degeneracy loci - an introduction to [P-R 2-5].

"explicit" is not necessary EXPLICIT!

In this section we summarize results and techniques mainly from [P-R 2-5] and [R] as well as from preceding papers $[\mathrm{H}-\mathrm{B}]$ and $[\mathrm{P} 4$, Sect. 6]. This section should serve as a "friendly" introduction to the papers [P-R 2-5] by J. Ratajski and the author, and convince the reader that this series of very technical - at the very first glance - papers relies on a childishly simple idea!

Let $G$ be a semisimple algebraic group, $B \subset G$ - a Borel subgroup and $T \subset B$ a maximal torus. We have the characteristic map

$$
c: S^{\bullet}(X(T)) \rightarrow A^{*}(G / B) .
$$


(see Section 4) which allows one to study the multiplicative structure of $A^{*}(G / B)$. On the other hand, looking at the additive structure, one has the Bruhat-Schubert cycles $X_{w}=\left[\overline{B^{-} w B / B}\right] \in A^{l(w)}(G / B)$. Here $w$ runs over the Weyl group $W$ and $B^{-}$denotes the Borel subgroup opposite to $B$. Since the Bruhat-Schubert cells $B^{-} w B / B(w \in W)$ form a cellular decomposition of $G / B$, one has $A^{*}(G / B)=\bigoplus_{w \in W} \mathbb{Z} X_{w}$.

The key problem is now to understand the coefficients $c_{w}(f)$ appearing in the decomposition

$$
c(f)=\sum_{w \in W} c_{w}(f) X_{w},
$$

where $f \in S^{\bullet}(X(T))$. This question is answered using the divided-differences operators $\partial_{w}$ which were defined in Section 3.

Theorem 6.1 [B-G-G], [D1,2]. If $f \in S^{\bullet}(X(T))$ is homogeneous then $c(f)=\sum_{l(w)=\operatorname{deg} f} \partial_{w}(f) X_{w}$. Moreover, the kernel of $c$ is generated by the positive degree $W$-invariants and $c \otimes \mathbb{Q}$ is surjective.

Observe that $\partial_{w}(\operatorname{Ker} c) \subset \operatorname{Ker} c$, so $\partial_{w}$ acts also on $A^{*}(G / B)$. One has (loc.cit.):

$$
\partial_{w} X_{v}= \begin{cases}X_{v w^{-1}} & \text { if } \quad l\left(v w^{-1}\right)=l(v)-l(w) \\ 0 & \text { otherwise. }\end{cases}
$$

In particular, $X_{w}=\partial_{w_{0} w^{-1}} X_{w_{0}}$ where $w_{0} \in W$ is the longest element in $W$. One has (loc.cit.):

$$
X_{w_{0}}=c\left(\prod_{\alpha \in R^{+}} \alpha /|W|\right)=c\left(\rho^{N} / N !\right),
$$

where $R^{+}$is the set of positive roots with respect to $B$ of cardinality $N$ and $\rho$ denotes the half of the sum of positive roots.

A similar theory works for the parabolic subgroups. Let $\Delta$ be a basis of the root system associated with $T$ and let $P$ be the parabolic subgroup associated with a subset $\theta \subset \Delta$. Denote by $W^{\theta}$ the set

$$
W^{\theta}=\left\{w \in W \mid l\left(w s_{\alpha}\right)=l(w)+1 \quad \forall \alpha \in \theta\right\} .
$$

The latter set is the set of minimal length left coset representatives of $W_{\theta}$, the subgroup of $W$ generated by $\left\{s_{\alpha}\right\}_{\alpha \in \theta}$, in $W$. (This follows, e.g., from the following fact: for every $w \in$ $W$, there exist unique $w^{\theta} \in W^{\theta}, w_{\theta} \in W_{\theta}$ such that $w=w^{\theta} w_{\theta}$ and $l(w)=l\left(w^{\theta}\right)+l\left(w_{\theta}\right)-$ see [Bou].) The projection $G / B \rightarrow G / P$ induces an inclusion $A^{*}(G / P) \rightarrow A^{*}(G / B)$ which (additively) identifies $A^{*}(G / P)$ with $\bigoplus_{w \in W^{\theta}} \mathbb{Z} X_{w}$. Multiplicatively, $A^{*}(G / P)$ is identified with the ring of invariants $A^{*}(G / B)^{W_{\theta}}$. Indeed, $X_{w} \in A(G / B)^{W_{\theta}}$ iff $\partial_{\alpha} X_{w}=0$ for all $\alpha \in \theta$. This takes place iff $l\left(w s_{\alpha}\right) \neq l(w)-1$, i.e. $l\left(w s_{\alpha}\right)=l(w)+1$, for all $\alpha \in \theta$; or equivalently $w \in W^{\theta}$. The restriction

$$
c: S^{\bullet}(X(T))^{W_{\theta}} \rightarrow A^{*}(G / P)
$$

of the characteristic map satisfies, for a homogeneous $f \in S^{\bullet}(X(T))$,

$$
c(f)=\sum_{\substack{w \in W^{\theta} \\ l(w)=\operatorname{deg} f}} \partial_{w}(f) X_{w} .
$$


The series of papers [P-R 2-5] deals with the case when $G$ is a classical group and $P$ is a maximal parabolic subgroup, i.e. $\theta=\Delta \backslash\{\alpha\}$ where $\alpha$ is a simple root. In every case like that, there exists a collection of "special" Schubert cycles $\left\{X_{p}\right\}$ forming a minimal set of multiplicative generators of $A^{*}(G / P)$ and a collection $\left\{e_{p}\right\}$ of elements in $S^{\bullet}(X(T))$ such that $c\left(e_{p}\right)=X_{p}$ up to a scalar. We specify these families "type by type": Let $S=S^{\bullet}(X(T))^{W_{\theta}} / \operatorname{Ker} c$. We use here the "Bourbaki notation" [Bou] for roots although denoting by $A_{n}$ the sequence of variables $\left(a_{1}, a_{2}, \ldots, a_{n}\right)$ where $\left(a_{1}, \ldots, a_{m}\right)$ is the sequence of "basic coordinates" and $n \leqslant m$. By $s_{i}$ we denote the simple reflection associated with the $i$-th simple root $\alpha_{i}$ specified below (in all types); it should not be confused with the Schur (or Segre) polynomial.

$\mathbf{A}_{m-1} \quad$ Simple roots: $\alpha_{n}=\varepsilon_{n}-\varepsilon_{n+1}, n=1, \ldots, m-1$.

For a fixed $n, W_{\alpha_{n}}=S_{n} \times \operatorname{id}_{S_{m-n}} \hookrightarrow S_{m}=W$;

$$
\begin{aligned}
& S=S \mathcal{P}\left(A_{n}\right) /\left(s_{j}\left(A_{n}\right) \mid m-n+1 \leqslant j \leqslant m\right) ; e_{p}=e_{p}\left(A_{n}\right) \\
& X_{p}=X_{s_{n+p-1} s_{n+p-2} \ldots s_{n}} \text { for } p=1, \ldots, n . \text { One has } c\left(e_{p}\right)=X_{p} .
\end{aligned}
$$

(Here, $G / P=G_{n}\left(\mathbb{C}^{m}\right)$.)

$\mathbf{B}_{m} \quad$ Simple roots: $\alpha_{n}=\varepsilon_{n}-\varepsilon_{n+1}(n<m), \alpha_{m}=\varepsilon_{m}$.

For a fixed $n, W_{\alpha_{n}}=S_{n} \times\left(S_{m-n} \ltimes \mathbb{Z}_{2}^{m-n}\right) \hookrightarrow S_{m} \ltimes \mathbb{Z}_{2}^{m}=W$ (W is identified with the group of "barred permutations" - see [H-B], [P-R2])

$$
\begin{aligned}
& S=S \mathcal{P}\left(A_{n}\right) /\left(s_{j}\left(a_{1}^{2}, \ldots, a_{n}^{2}\right) \mid m-n+1 \leqslant j \leqslant m\right) ; e_{p}=e_{p}\left(A_{n}\right), \\
& X_{p}=X_{s_{n-p+1} s_{n-p+2} \ldots s_{n}} \text { for } p=1, \ldots, n . \text { For } m=n \text { one has } c\left(e_{p}\right)=2 X_{p} .
\end{aligned}
$$

For $n<m, c\left(e_{p}\right)=X_{p}$.

(Here, $G / P=O G_{n}\left(\mathbb{C}^{2 m+1}\right)$ is the Grassmannian of isotropic $n$-subspaces in $\mathbb{C}^{2 m+1}$ endowed with a symmetric nondegenerate form.)

$\mathbf{C}_{m} \quad$ Simple roots: $\alpha_{n}=\varepsilon_{n}-\varepsilon_{n+1}(n<m), \alpha_{m}=2 \varepsilon_{m}$.

For a fixed $n, W_{\alpha_{n}}=S_{n} \times\left(S_{m-n} \ltimes \mathbb{Z}_{2}^{m-n}\right) \hookrightarrow S_{m} \ltimes \mathbb{Z}_{2}^{m}=W$;

$$
\begin{aligned}
& S=S \mathcal{P}\left(A_{n}\right) /\left(s_{j}\left(a_{1}^{2}, \ldots, a_{n}^{2}\right) \mid m-n+1 \leqslant j \leqslant m\right) ; e_{p}=e_{p}\left(A_{n}\right), \\
& X_{p}=X_{s_{n-p+1} s_{n-p+2} \ldots s_{n}} \text { for } p=1, \ldots, n . \text { One has } c\left(e_{p}\right)=X_{p} .
\end{aligned}
$$

(Here, $G / P=L G_{n}\left(\mathbb{C}^{2 m}\right)$ is the Lagrangian Grassmannian of isotropic $n$-subspaces in $\mathbb{C}^{2 m}$ endowed with an antisymmetric nondegenerate form.)

$\mathbf{D}_{m} \quad$ Simple roots: $\alpha_{n}=\varepsilon_{n}-\varepsilon_{n+1}(n<m), \alpha_{m}=\varepsilon_{m-1}+\varepsilon_{m}$.

For a fixed $n, W_{\alpha_{n}}=S_{n} \times\left(S_{m-n} \ltimes \mathbb{Z}_{2}^{m-n-1}\right) \hookrightarrow S_{m} \ltimes \mathbb{Z}_{2}^{m-1}=W$, the latter being identified with the group of "even-barred permutations";

For $n=m, e_{p}=e_{p}\left(A_{n}\right), p=1, \ldots, n-1$, generate multiplicatively $S$; for $n<m$, $e_{p}\left(A_{n}\right), p=1, \ldots, n$ and $a_{n+1} \cdot \ldots \cdot a_{m}$ generate multiplicatively $S$.

We propose the following choice of special Schubert cycles: $X_{p}=X_{s_{n-p+1} s_{n-p+2} \ldots s_{n}}$ for $p=1, \ldots, n$ and additionally for $n<m, X_{0}=X_{s_{n} s_{n+1} \ldots s_{m-1}}$. One has $c\left(e_{p}\right)=X_{p}$ for $p=1, \ldots, n$ and, for $n<m, c\left(a_{n+1} \cdot \ldots \cdot a_{m}\right)=X_{0}$.

(Here, $G / P=O G_{n}\left(\mathbb{C}^{2 m}\right)$ is the Grassmannian of isotropic $n$-subspaces in $\mathbb{C}^{2 m}$ endowed with a symmetric nondegenerate form. Recall that for $n<m, G / P=O G_{n}\left(\mathbb{C}^{2 m}\right)$ is irreducible, and for $n=m$, it has two connected components.) 
Our main task here are Pieri-type formulas, i.e. we wish to give explicit formulas for the coefficients $m_{w, p}^{v} \in \mathbb{N}$ appearing in the equations:

$$
X_{w} \cdot X_{p}=\sum m_{w, p}^{v} X_{v}
$$

where $w, v \in W^{\theta}(\theta=\Delta \backslash\{\alpha\}, \alpha$ a simple root). For the spaces $G / B$, there exists a formula of this type due to $\mathrm{C}$. Chevalley $[\mathrm{Ch}]\left({ }^{14}\right)$ :

$$
X_{w} \cdot X_{s_{\alpha}}=\sum\left(\beta^{\vee}, \omega_{\alpha}\right) X_{w s_{\beta}}
$$

where $w \in W$ and $\beta$ runs over positive roots such that $l\left(w s_{\beta}\right)=l(w)+1$. One can use the Chevalley formula to compute the Pieri-type formulas for $G / P$ in question. This method leads, however, to very complicated computations (compare [H-B]) which make it practically unaccessible for the Grassmannians of non-maximal subspaces of types $B$, $C, D$.

The strategy taken in [P-R 2-4] relies on a totally different method; namely, it uses an iteration of the following Leibniz-type formula: for $f, g \in S^{\bullet}(X(T))$ and a root $\alpha$, one has:

$$
\partial_{\alpha}(f \cdot g)=f \cdot\left(\partial_{\alpha} g\right)+\left(\partial_{\alpha} f\right) \cdot\left(s_{\alpha} g\right) .
$$

More precisely, the calculation goes as follows. Let $f$ be a homogeneous element of $S^{\bullet}(X(T)) \otimes \mathbb{Q}$ such that $c(f)=X_{w}$. We have for a reduced decomposition $v=s_{\alpha_{1}} \ldots \cdot s_{\alpha_{k}}$,

$$
\begin{aligned}
m_{w, p}^{v} & =\partial_{v}\left(f \cdot e_{p}\right)=\partial_{\alpha_{1}} \circ \cdots \circ \partial_{\alpha_{k}}\left(f \cdot e_{p}\right)= \\
& =\sum \partial_{I}(f) \cdot \partial_{\alpha}^{I}\left(e_{p}\right),
\end{aligned}
$$

where the sum is over all subsequences $I=\left(i_{1}<\cdots<i_{l}\right) \subset\{1, \ldots, k\} ; \partial_{I}$ is $\partial_{r_{I}}$ where $r_{I}=s_{\alpha_{i_{1}}} \cdot \ldots \cdot s_{\alpha_{i_{k}}}$ and $\partial_{\alpha}^{I}$ is obtained by replacing in $\partial_{\alpha_{1}} \circ \cdots \circ \partial_{\alpha_{k}}$ the subword $\partial_{I}$ through the subword $r_{I}$. By the choice of $f$ we finally infer

LEMMA 6.2. $m_{w, p}^{v}=\sum \partial_{\alpha}^{I}\left(e_{p}\right)$, where the sum runs over $I$ such that $r_{I}$ is a reduced decomposition of $w$ (notation: $\left.r_{I} \in R(w)\right)$.

The content of papers [P-R 2-4] and $[\mathrm{R}]$ can be summarized, in a coarse way, in the following theorem.

THEOREM 6.3 [P-R 2-4], [R]. In the above notation, for types $A_{m-1}, B_{m}, C_{m}\left({ }^{15}\right)$ and any simple root $\alpha$, the following assertions hold $\left(W^{\alpha}=W^{\Delta \backslash\{\alpha\}}\right)$ :

I. For any $v \in W^{\alpha}$ there exists a reduced decomposition $v=s_{\alpha_{1}} \cdot \ldots \cdot s_{\alpha_{k}}$ such that for any $w \in W^{\alpha}$ with $k=l-p$, the cardinality of the set

$$
\mathcal{I}=\left\{I \subset\{1, \ldots, k\} \mid s_{\alpha_{i_{1}}} \cdot \ldots \cdot s_{\alpha_{i_{l}}} \in R(w) \text { and } \partial_{\alpha}^{I}\left(e_{p}\right) \neq 0\right\}
$$

is less than or equal to 1.

II. There exists an EXPLICIT combinatorial criterion for card $\mathcal{I}=1$.

III. If card $\mathcal{I}=1$ then the unique $I=I(\alpha ., w) \in \mathcal{I}$ is given by an EXPLICIT combinatorial algorithm.

IV. The multiplicity is given by the formula $m_{w, p}^{v}=\partial_{\alpha .}^{I}\left(e_{p}\right)=2^{e(w, v)}$, where $e(w, v)$ is given by an EXPLICIT combinatorial rule.

$\left({ }^{14}\right)$ Note added in proof: This paper, written about 1958, has been published only recently.

$\left({ }^{15}\right)$ Note added in proof: The authors have recently extended the result also to type $D_{m}$. See a forthcoming paper: P. Pragacz and J. Ratajski, A Pieri-type theorem for even orthogonal Grassmannians. 
We now illustrate the theorem for type $C_{n}$ and the simple root $\alpha_{n}$. Let, for the rest of this section, $\mathcal{G}=L G_{n}\left(\mathbb{C}^{2 n}\right)$ denote the Lagrangian Grassmannian of $n$-dimensional isotropic subspaces in $\mathbb{C}^{2 n}$ with respect to a nondegenerate antisymmetric form on $\mathbb{C}^{2 n}$. Let $\mathcal{F}$ denote the flag variety of (total) isotropic flags in $\mathbb{C}^{2 n}$ (with respect to the same antisymmetric form). By $\rho_{n}$ we denote the partition $(n, \ldots, 2,1)$. Let $I=\left(i_{1}>i_{2}>\right.$ $\left.\cdots>i_{k}>0\right) \subset \rho_{n}$ be a strict partition. We associate with $I$ the element $w_{I}$ of the symplectic Weyl group $W\left({ }^{16}\right)$ :

$$
w_{I}=\left(s_{n-i_{k}+1} \ldots s_{n-1} s_{n}\right) \ldots\left(s_{n-i_{2}+1} \ldots s_{n-1} s_{n}\right)\left(s_{n-i_{1}+1} \ldots s_{n-1} s_{n}\right) .
$$

From the theory described above we get a Schubert cycle $X_{w_{I}} \in A^{|I|}(\mathcal{F})$, defined as the class of the closure of $B^{-} w_{I} B / B$ where $B$ is the Borel subgroup of the symplectic group and $B^{-}$- its opposite. In fact, $X_{w_{I}}$ belongs to $A^{|I|}(\mathcal{G}) \subset A^{|I|}(\mathcal{F})$. Denote this element in $A^{|I|}(\mathcal{G})$ by $\sigma(I)$, for brevity.

As usual, we associate to a partition $I$ its Ferrers' diagram $D_{I}$ (see [M1]) and treat it as a subset of $\mathbb{Z} \times \mathbb{Z}$. A subset $D \subset \mathbb{Z} \times \mathbb{Z}$ is connected if each of the sets $\{i: \exists j(i, j) \in D\}$ and $\{j: \exists i(i, j) \in D\}$ is an interval in $\mathbb{Z}$. This allows us to speak about the "connected components" of skew diagrams, i.e. the differences between diagrams of partitions.

The following result was given originally in [H-B] and reproved in [P-R2].

TheOREM $6.4[\mathrm{H}-\mathrm{B}]$. Let $I=\left(i_{1}, \ldots, i_{k}\right) \subset \rho_{n}$ be a strict partition. The following equality holds in $A^{*}(\mathcal{G})(p=1, \ldots, n)$ :

$$
\sigma(I) \sigma(p)=\sum 2^{e(I, J)} \sigma(J)
$$

where the sum is over strict partitions $J$ such that $i_{h-1} \geqslant j_{h} \geqslant i_{h}\left(i_{0}=n, i_{k+1}=0\right)$, $|J|=|I|+p$ and $e(I, J)$ is the number of connected components of $D_{J} \backslash D_{I}$ not meeting the first column.

(This formulation of the theorem is slightly different from the one in $[\mathrm{H}-\mathrm{B}]$ and is suited to the techniques of $[\mathrm{P}-\mathrm{R} 2]$.)

EXAMPLE 6.5. $n=7$

$$
\sigma(632) \sigma(5)=2 \sigma(763)+2^{2} \sigma(7531)+2 \sigma(7621)+2 \sigma(7432)+\sigma(6532) .
$$
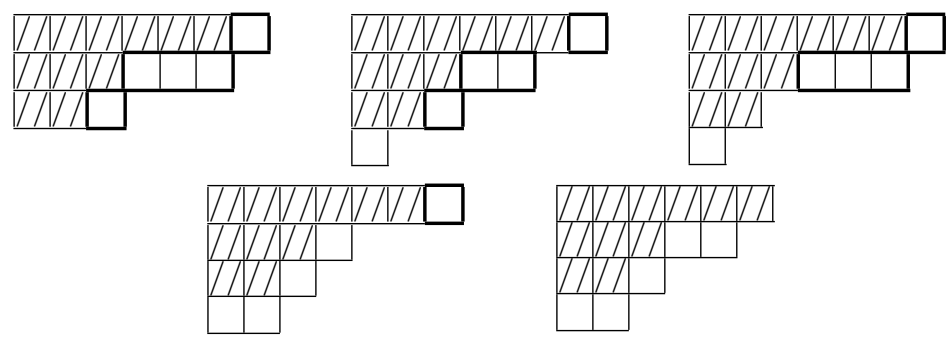

We now sketch a proof of this theorem, in the spirit of Theorem 6.3 above, stemming from $[\mathrm{P}-\mathrm{R} 2]$. This proof is much simpler than the one in $[\mathrm{H}-\mathrm{B}]$ based on the Chevalley formula.

Let $A=\left(a_{1}, \ldots, a_{n}\right)$ be a sequence of independent variables. It follows from [B-G-G] and $[\mathrm{D} 1,2]$ that $A^{*}(\mathcal{F})$ is identified with $\mathbb{Z}[A] / \mathcal{I}$, where $\mathcal{I}$ is the ideal generated by symmetric polynomials in $a_{1}^{2}, \ldots, a_{n}^{2}$ without constant term. Also, $A^{*}(\mathcal{G})$ is identified with

$\left({ }^{16}\right)$ Footnote 10 applies here with $S_{n}$ replaced by $W$. 
$(\mathbb{Z}[A] / \mathcal{I})^{S_{n}}$, i.e. with the quotient of the symmetric polynomials modulo $\mathcal{I}$ restricted to the ring of symmetric polynomials.

We have "symplectic divided differences": $\partial_{i}: \mathbb{Z}[A] \rightarrow \mathbb{Z}[A]$ (of degree -1 ), $i=$ $1, \ldots, n$, defined by

$$
\partial_{i}(f)=\left(f-s_{i} f\right) /\left(a_{i}-a_{i+1}\right), \quad i=1, \ldots, n-1, \quad \partial_{n}(f)=\left(f-s_{n} f\right) / 2 a_{n} .
$$

Recall that there exists a surjective characteristic map $c: \mathbb{Z}[A] \rightarrow A^{*}(\mathcal{F})$, and $c\left(e_{p}\right)=$ $\sigma(p)=X_{p} \in A^{p}(\mathcal{G})([\mathrm{H}-\mathrm{B}])$.

Let $f_{I} \in \mathbb{Z}[A]$ be homogeneous such that $c\left(f_{I}\right)=\sigma(I)$. Then, for $w \in W, l(w)=|\lambda|$, we have $\partial_{w}\left(f_{I}\right) \neq 0$ iff $w=w_{I}$ and $\partial_{w_{I}}\left(f_{I}\right)=1$. Following Theorem 6.3 , our goal is to find the coefficients $m_{J}$ appearing in

$$
c\left(f_{I} \cdot e_{p}\right)=\sum m_{J} \sigma(J) .
$$

Consider an arbitrary subset $D \subset D_{J}$. (Subsequences $I$ in Lemma 6.2 and Theorem 6.3 correspond here to subsets $D$ of $D_{J}$.) The boxes in $D_{J}$ which belong to $D$ will be called $D$-boxes; the boxes in $D_{J} \backslash D$ will be called non-D-boxes. We associate with $D$ the word $r_{D}$ and the operator $\partial_{J}^{D}$, given by Definitions 6.6 and 6.7. For technical reasons we will use, from now on, the "matrix" coordinates for indexing boxes in $D_{J} \subset \rho_{n}$ but the columns are numbered from left to right by $n, n-1, \ldots, 2,1$ successively.

In Definitions 6.6, 6.7 we read $D_{J}$ like books (e.g., in Europe), i.e. row by row from left to right starting from the top row.

Definition 6.6 of $r_{D}$. Read $D_{J}$. Every $D$-box in the $i$-th column gives us the $s_{i}$. Non- $D$-boxes have no influence on $r_{D}$. Then $r_{D}$ is the word obtained by writing the $s_{i}$ 's from right to left.

Definition 6.7 of $\partial_{J}^{D}$. Read $D_{J}$. Every $D$-box in the $i$-th column gives us the $s_{i}$. Every non- $D$-box in the $i$-th column gives the $\partial_{i}$. Then $\partial_{J}^{D}$ is the composition of the $s_{i}$ 's and $\partial_{i}$ 's (the composition written from right to left).

EXAMPLE 6.8. $J=(763), n=7$.

$$
\begin{array}{lllllll}
7 & 6 & 5 & 4 & 3 & 2 & 1
\end{array}
$$

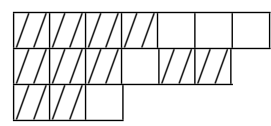

( $D$-boxes are shaded here)

$r_{D}=s_{6} \cdot s_{7} \cdot s_{2} \cdot s_{3} \cdot s_{5} \cdot s_{6} \cdot s_{7} \cdot s_{4} \cdot s_{5} \cdot s_{6} \cdot s_{7}$,

$\partial_{J}^{D}=\partial_{5} \circ s_{6} \circ s_{7} \circ s_{2} \circ s_{3} \circ \partial_{4} \circ s_{5} \circ s_{6} \circ s_{7} \circ \partial_{1} \circ \partial_{2} \circ \partial_{3} \circ s_{4} \circ s_{5} \circ s_{6} \circ s_{7}$.

In the above notation, Lemma 6.2 reads as follows: $m_{J}=\sum \partial_{J}^{D}\left(e_{p}\right)$, where the sum is over all $D \subset D_{J}$ such that $r_{D} \in R\left(w_{I}\right)$.

One proves that if $j_{h+1}>i_{h}$ for some $h$ (in particular if $l(J)>l(I)+1$ ) then $\partial_{J}^{D}\left(\sum e_{p}\right)=0$ for every $D \subset D_{J}$ such that $r_{D} \in R\left(w_{I}\right)$.

Moreover, fix a strict partition $I \subset \rho_{n}$. Let $J$ be a strict partition such that $I \subset J \subset$ $\rho_{n}, j_{h+1} \leqslant i_{h}$ for every $h$ (in particular $\left.l(J) \leqslant l(I)+1\right)$. Then there exists exactly one $D^{I, J} \subset D_{J}$ such that $r_{D} \in R\left(w_{I}\right)$ and $\partial_{J}^{D}\left(\sum e_{p}\right) \neq 0$ for $D=D^{I, J}$.

The idea of constructing such a $D^{I, J}$ can be easily explained using pictures. The boxes from $D_{I} \subset D_{J}$ are shaded in the pictures below. A part: 


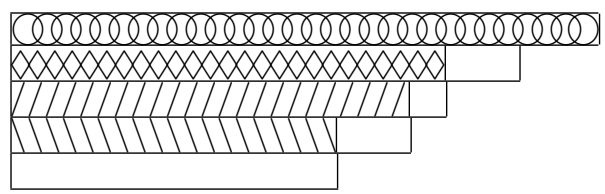

of the diagram $D_{I} \subset D_{J}$ is deformed to:

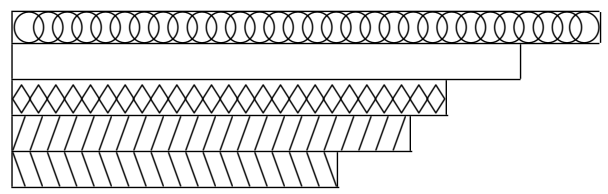

On the other side, a part:

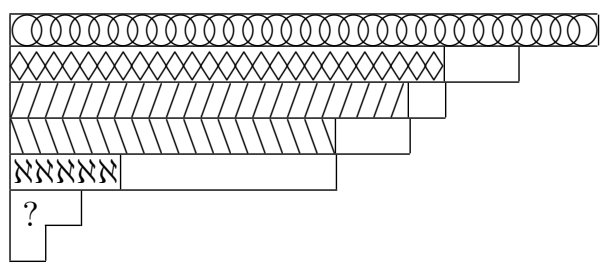

of the diagram $D_{I} \subset D_{J}$ is deformed to:

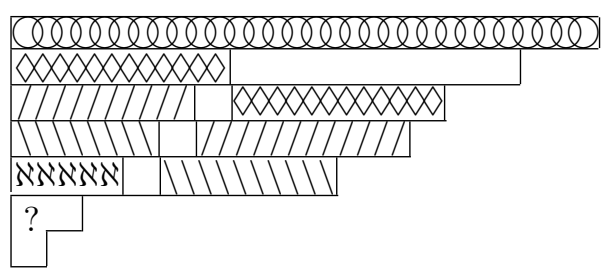

The deformations are performed in direction South $\rightarrow$ North.

Fix a strict partition $I \subset \rho_{n}$ and a number $p=1, \ldots, n$. Let $J$ be a strict partition such that $I \subset J \subset \rho_{n},|J|=|I|+p, j_{h+1} \leqslant i_{h}$ for every $h$. Let $D=D^{I, J}$. Every $\partial_{i}$ involved in $\partial_{J}^{D}$ is associated with a box in $D_{J} \backslash D$. It turns out that the connected components of $D_{J} \backslash D$ play a crucial role in the computation of $\partial_{J}\left(e_{p}\right)$. Namely, in the above notation,

$$
\partial_{J}^{D}\left(e_{p}\right)=2^{e(I, J)}
$$

where $e(I, J)$ is the number of connected components of $D_{J} \backslash D$ not meeting the $n$-th column. By changing the numbering of columns to the usual ordering, this can be easily restated as: $e(I, J)$ is the number of connected components of $D_{J} \backslash D_{I}$ not meeting the first column. 
This finishes the sketch of the proof of Theorem 6.4 in the spirit of Theorem $6.3-$ for more details see [P-R2].

ExAMPLE 6.9. The diagrams $D^{(632), J}$ for partitions $J$ appearing in the decomposition $\sigma(632) \sigma(5)$, are:
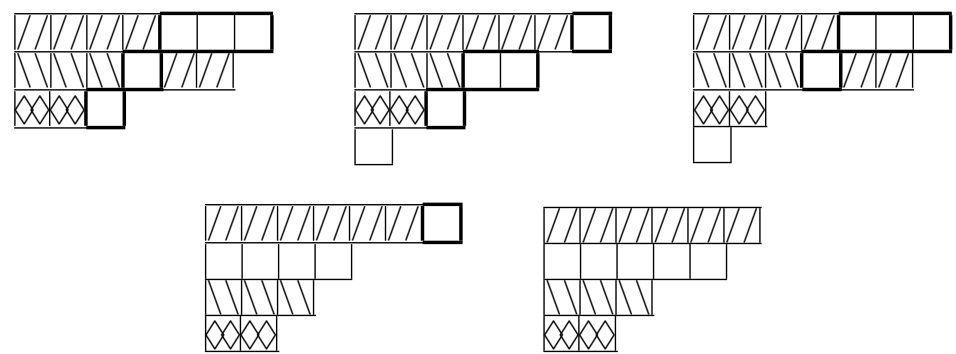

A geometric interpretation of $\sigma(I)$ is as follows. Let $V$ be a vector space of dimension $2 n$ endowed with an antisymmetric nondegenerate form $\phi: V \times V \rightarrow \mathbb{C}$. Let $\left(v_{1}, \ldots, v_{n}\right)$ be a basis of an isotropic $n$-subspace of $V$. Let $V_{1} \subset V_{2} \subset \cdots \subset V_{n}$ be a flag of isotropic subspaces where $V_{i}$ is spanned by the first $i$ vectors in the sequence $\left(v_{1}, \ldots, v_{n}\right)$. Then $\sigma\left(i_{1}, \ldots, i_{k}\right)$ is the class in $A^{|I|}(\mathcal{G})$ of the cycle of all isotropic $n$-subspaces $L$ in $V$ such that

$$
\operatorname{dim}\left(L \cap V_{n+1-i_{h}}\right) \geqslant h, h=1, \ldots, k .
$$

A connection with Q-polynomials. In $[\mathrm{P} 4]$ the author has deduced from Theorem 6.4 the following result, where $Q_{I}$ denotes the Schur $Q$-polynomial (see Section 1):

TheOREM 6.10 [P4, Sect. 6]. The assignment $Q_{I} \mapsto \sigma(I)$ for $I \subset \rho_{n}-$ zero otherwise, defines a ring homomorphism and allows one to identify $A^{*}(\mathcal{G})$ with the factor of the ring of $Q$-polynomials modulo the ideal $\bigoplus \mathbb{Z} Q_{I}$, the sum over all $I$ not contained in $\rho_{n}$.

In particular the following Giambelli-type formula is valid for the Schubert cycle $\sigma(I)$.

TheOREM 6.11 [P4, Sect. 6]. Let $I=\left(i_{1}, \ldots, i_{k}\right) \subset \rho_{n}$ be a strict partition, $k$-even (we can always assume it by putting $i_{k}=0$, if necessary). Then

$$
\sigma(I)=\operatorname{Pfaffian}\left[\sigma\left(i_{p}, i_{q}\right)\right]_{1 \leqslant p, q \leqslant k},
$$

where, for $p<q, \sigma\left(i_{p}, i_{q}\right)=\sigma\left(i_{p}\right) \sigma\left(i_{q}\right)+2 \sum_{h=1}^{q}(-1)^{h} \sigma\left(i_{p}+h\right) \sigma\left(i_{q}-h\right)$, and $\sigma\left(i_{p}, 0\right)=$ $\sigma\left(i_{p}\right)$

With the use of the tautological Lagrangian (sub)bundle $R$ on $\mathcal{G}$, this result is rewritten as $\sigma(I)=\widetilde{Q}_{I} R^{\vee}$ (see Section 4 for the definition of the right-hand side).

Schubert calculus for usual Grassmannians is based on three main theorems: Pieri's formula, Giambelli determinantal formula and the basis theorem. Analogues of the formulas are given in Theorems 6.4 and 6.11. A basis-type theorem for the Lagrangian Grassmannian $\mathcal{G}$ can be formulated as

Theorem 6.12. $A_{*}(\mathcal{G})=\bigoplus \mathbb{Z} \sigma(I)$, where the sum is over all strict partitions $I \subset \rho_{n}$.

This result globalizes to Lagrangian Grassmannian bundles $L G_{n}(V) \rightarrow X$ (the notation as in Section 4). One has $A^{*}\left(L G_{n}(V)\right)=\bigoplus A^{*}(X) \widetilde{Q}_{I} R^{\vee}$, the sum over all strict partitions $I \subset \rho_{n}$. 
Theorem 6.12 can be deduced from a general theory of the cellular Schubert-Bruhat decompositions of homogeneous spaces (see, e.g., [Ch], [B-G-G], [D2]). The cellular decomposition in the case of $\mathcal{G}$ was described in details in [P4, Sect. 6]. Another simple, conceptual proof of Theorem 6.12 is given in [P-R2].

Using similar arguments one can prove a Pieri-type formula for the Grassmannian of $n$-dimensional isotropic subspaces of $(2 n+1)$-dimensional vector space endowed with a symmetric nondegenerate form (a Pieri-type formula in this case was originally given in $[\mathrm{H}-\mathrm{B}])$. A connection with P-polynomials and a Giambelli-type formula were originally established in [P4, Sect. 6].

Analogous results in the case of Grassmannian of $n$-dimensional isotropic subspaces in a $2 n$-dimensional vector space endowed with a symmetric nondegenerate form were worked out in [P4, Sect. 6].

The Giambelli-type formulas for isotropic Grassmannians described above are globalized in [P-R5] to Lagrangian and orthogonal degeneracy loci. In particular, the following problem is solved:

Problem (J. Harris). Let $V$ be a vector bundle over $X$ equipped with a nondegenerate antisymmetric or symmetric form and let $E, F$ be two maximal isotropic subbundles of $V$. Express the fundamental class of the locus

$$
D^{k}=\{x \in X \mid \operatorname{dim}(E(x) \cap F(x)) \geqslant k\}
$$

as a polynomial in the Chern classes of the bundles involved. (For a definition of an appropriate scheme structure on $D^{k}$ - see [P-R5].)

Observe that if $X=L G_{n}\left(\mathbb{C}^{2 n}\right), E$ is the tautological vector bundle on $X$ and $F$ is a trivial isotropic rank $n$ bundle on $X$, then the fundamental class of the above locus is the Schubert cycle $\sigma(k, k-1, \ldots, 1)$. By the above, this Schubert cycle is expressed by $\widetilde{Q}_{(k, k-1, \ldots, 1)} E^{\vee}$.

More generally, one has:

THEOREM 6.13 [P-R5]. Let $V$ be a vector bundle over a pure-dimensional CohenMacaulay variety endowed with a nondegenerate antisymmetric form. Let $E, F \subset V$ be two maximal isotropic subbundles. If $D^{k}$ is of pure codimension $k(k+1) / 2$ in $X$ or empty, then

$$
\left[D^{k}\right]=\sum_{I \subset \rho_{k}} \widetilde{Q}_{I} E^{\vee} \cdot \widetilde{Q}_{\rho_{k} \backslash I} F^{\vee},
$$

where $\rho_{k}=(k, k-1, \ldots, 1)$ and for a strict $I \subset \rho_{k}, \rho_{k} \backslash I$ is the strict partition whose parts complement $I$ in $\{k, k-1, \ldots, 1\}\left({ }^{17}\right)$.

There exists $([\mathrm{P}-\mathrm{R} 5])$ an analogue of this theorem for the symmetric form; this analogue has allowed recently a computation of the cohomology class of Brill-Noether loci in Prym varieties. Let $K$ be an algebraically closed field of characteristic different from

$\left({ }^{17}\right)$ Note added in proof: A different (in its form) solution to Harris' problem has been obtained independently by W. Fulton in Determinantal Formulas for Orthogonal and Symplectic Degeneracy Loci - to appear in J. Differential Geom. and Schubert Varieties in Flag Bundles for the Classical Groups - to appear in "Hirzebruch 65", Israel Math. Conf. Proc. Both the papers depend on the paper by D. Edidin and W. Graham, Characteristic classes and quadric bundles — Duke Math. J. 78 (1995), 277-299. 
2. Let $\pi: \widetilde{C} \rightarrow C$ be an étale double cover of a nonsingular algebraic curve $C$ over $K$ of genus $g$ (hence the genus of $\widetilde{C}$ is $2 g-1$ ). Let $\mathrm{Nm}: \operatorname{Pic}^{2 g-2}(\widetilde{C}) \rightarrow \operatorname{Pic}^{2 g-2}(C)$ be the norm map associated with $\pi$; the scheme $\mathrm{Nm}^{-1}\left(\omega_{C}\right), \omega_{C}$ being the canonical class, breaks up into two connected components $P^{+}$and $P^{-}$corresponding to even or odd values of $h^{0}(-)$. These components, being translates of the Prym variety associated with $\pi$, are irreducible of dimension $g-1$. The following definition of the closed subsets $V^{r}$ in $P^{ \pm}$ (called "Brill-Noether loci for Pryms") is due to G. Welters. For every integer $r \geqslant-1$ one sets:

$$
V^{r}=\left\{L \in \mathrm{Nm}^{-1}\left(\omega_{C}\right) \mid h^{0}(L) \geqslant r+1 \text { and } h^{0}(L) \equiv r+1(\bmod 2)\right\} .
$$

TheOREm 6.14 [DC-P]. Assume that $V^{r}$ either is empty or has pure codimension in $P^{ \pm}$equal to $r(r+1) / 2$. Then its class in the numerical equivalence ring of $P^{ \pm}$, or its cohomology class in $H^{*}\left(P^{ \pm}, \mathbb{C}\right) \quad($ for $K=\mathbb{C})$, is equal to

$$
2^{r(r-1) / 2} \prod_{i=1}^{r} \frac{(i-1) !}{(2 i-1) !} \xi^{r(r+1) / 2} .
$$

where $\xi$ is the class of the theta divisor on $P^{ \pm}$.

The assumptions of the theorem, as shown by Welters, are satisfied for a general curve $C$ and any irreducible double cover $\pi: \widetilde{C} \rightarrow C$ of it. For more details, concerning, e.g., a definition of an appropriate scheme structure on $V^{r}$, see [DC-P] and the references therein.

The paper [P-R5] gives also formulas for more general loci; in the following $a$. is a sequence $a .=\left(1 \leqslant a_{1}<\cdots<a_{k} \leqslant n\right)$ :

$$
D(a .)=\left\{x \in X \mid \operatorname{dim}\left(E(x) \cap F_{a_{p}}(x)\right) \geqslant p, p=1, \ldots, k\right\}
$$

where $F_{\bullet}=F_{1} \subset F_{2} \subset \cdots \subset F_{n}=F$ is an isotropic flag. The fundamental classes of these loci are given in loc.cit. as quadratic expressions in $\widetilde{Q}$-polynomials of $E$ and flag Schur polynomials of $F_{\bullet}$ (i.e. the determinants of the matrices of the form $\left.\left[s_{i_{p}-p+q}\left(F_{p}\right)\right]_{p, q}\right)$.

By adapting the technique of Hodge and Pedoe, S. Sertöz has obtained in [Se] a "triple Pieri intersection theorem" for isotropic Grassmannians in the orthogonal case; however, the main result in $[\mathrm{P}-\mathrm{R} 3,4]$ cannot be deduced from [Se].

7. Numerically positive polynomials for ample vector bundles with applications to Schur polynomials of Schur bundles and a vanishing theorem.

Question: "Which result of algebraic geometry is the most useful for combinatorists?" Answer: "Perhaps ... Hard Lefschetz!"

In this section, we denote by $X$ a nonsingular projective variety. Let $L=\mathcal{O}_{X}(1)$. Suppose that $c_{1}, \ldots, c_{e}$ are independent variables such that $\operatorname{deg} c_{i}=i$. We say, following [F-L], that a polynomial $P \in \mathbb{Z}\left[c_{1}, \ldots, c_{e}\right], \operatorname{deg} P=d$, is numerically positive for ample vector bundles if for every $X$ of dimension $n \geqslant d$ and every ample vector bundle of rank $e$ on $X$, the number $P\left(c_{1}(E), \ldots, c_{e}(E)\right) \cdot c_{1}(L)^{n-d} \in A_{0}(X)=\mathbb{Z}$, is positive.

Recall (see Section 1) that the Schur polynomial associated with a partition $I$ is defined by

where $J=I^{\sim}$. One has

$$
s_{I}(c .)=\operatorname{Det}\left[c_{j_{p}-p+q}\right]_{1 \leqslant p, q \leqslant i_{1}},
$$


THEOREM 7.1 [F-L]. Every numerically positive polynomial is a $\mathbb{Z}$-combination $\sum d_{I} s_{I}(c$.$) , where all d_{I} \geqslant 0$ and $\sum d_{I}>0\left({ }^{18}\right)$.

This theorem, based on the Hard Lefschetz theorem and the Giambelli-Kempf-Laksov formula (i.e. the formula from Example 3.4), has a nice algebraic consequence. We follow the notation from Section 2.

The following observation is due to the author.

Corollary 7.2. Let $E_{1}, \ldots, E_{k}$ be vector bundles. Then for partitions $I ; J_{1}, \ldots, J_{k}$; $K_{1}, \ldots, K_{k}$, the coefficients $d\left(I ; J_{1}, \ldots, J_{k} ; K_{1}, \ldots, K_{k}\right)$ appearing in the decomposition

$$
\begin{aligned}
& \qquad s_{I}\left(S^{J_{1}} E_{1} \otimes \cdots \otimes S^{J_{k}} E_{k}\right)=\sum_{K .} d\left(I ; J_{1}, \ldots, J_{k} ; K_{1}, \ldots, K_{k}\right) s_{K_{1}}\left(E_{1}\right) \cdot \ldots s_{K_{k}}\left(E_{k}\right) \\
& \text { satisfy } d\left(I ; J_{1}, \ldots, J_{k} ; K_{1}, \ldots, K_{k}\right) \geqslant 0 \text { and } \sum_{K} d\left(I ; J_{1}, \ldots, J_{k} ; K_{1}, \ldots, K_{k}\right)>0 .
\end{aligned}
$$

Of course, the coefficients $d\left(I ; J_{1}, \ldots, J_{k} ; K_{1}, \ldots, K_{k}\right)$ are universal, i.e., they depend on $\operatorname{rank}\left(E_{1}\right), \ldots, \operatorname{rank}\left(E_{k}\right)$ only. Therefore, it is sufficient to show the assertion for ample bundles $E_{1}, \ldots, E_{k}$ with algebraically independent Chern roots. This can be achieved by taking as $X$ the product of Grassmannians

$$
X=\prod_{i=1}^{k} G^{e_{i}}\left(\mathbb{C}^{n_{i}}\right),
$$

where $e_{i}=\operatorname{rank} E_{i}$ and $n_{i} \gg 0$. Let $L=\mathcal{O}_{X}(1)$. Moreover, denote by $p_{i}$ the projection $X \rightarrow G^{e_{i}}\left(\mathbb{C}^{n_{i}}\right)$ and by $Q_{i}$ the tautological quotient bundle on $G^{e_{i}}\left(\mathbb{C}^{n_{i}}\right)$. Define $E_{i}:=$ $\left(p_{i}^{*} Q_{i}\right) \otimes L(i=1, \ldots, k)$. The bundles $E_{i}$ are ample. Hence $S^{J_{1}} E_{1} \otimes \cdots \otimes S^{J_{k}} E_{k}$ is ample (see $[\mathrm{Ha}])$. The assertion now follows from Theorem 7.1.

For example, the numbers $D_{I, J}^{m, n},((J))$ and $[J]$ from Section 2 are nonnegative.

It would be interesting to have a purely algebro-combinatorial proof of Corollary 7.2. Note that a combinatorial interpretation of the numbers $D_{I, J}^{m, n}$ was given in [G-V].

In [D-P-S], the authors show that the above Fulton-Lazarsfeld inequalities for Chern classes and Schur polynomials of ample vector bundles still hold for nef vector bundles on compact Kähler manifolds. Recall that a vector bundle $E$ is numerically effective if the Grothendieck line bundle $\mathcal{O}(1)$ on $G^{1}(E)$ is nef, i.e. has a nonnegative degree on each effective curve in $G^{1}(E)$.

As shown by L. Manivel in the next theorem, the numerical positivity of Schur polynomials of vector bundles can be used to extend vanishing theorems to wider classes of vector bundles.

THEOREM 7.3 [Ma]. Let $E$ be a numerically effective vector bundle on a projective variety $X$ of dimension $n$. Suppose that a line bundle $L$ on $X$ is nef and either there exists a partition I such that $\int_{X} s_{I}(E)>0$ or $L$ is "big" (or equivalently $\left.\int_{X} c_{1}(L)^{n}>0\right)$. Then, for every partition $J$ of length $l(J) \geqslant l(I)$,

$$
H^{q}\left(X, K_{X} \otimes S^{J} E \otimes(\operatorname{Det} E)^{l} \otimes L\right)=0
$$

$\left({ }^{18}\right)$ Note added in proof: W. Fulton has obtained recently an analogue of this result for filtered ample bundles where the role of Schur polynomials is played by Schubert polynomials, recalled in Sections 3 and 4. See: W. Fulton, Positive polynomials for ample vector bundles, Amer. J. Math. 117 (1995), 627-633. 
for $q>0$ and $l \geqslant l(J)$.

This result, extending the familiar Kawamata-Viehweg vanishing theorem, is derived from an expression of the self-intersection of a line bundle on a relative flag manifold. This expression is a consequence of some expansion of the Chern character of symmetric powers combined with the Hirzebruch-Riemann-Roch formula $[\mathrm{H}]$, and gives an insight into the corresponding Gysin map that seems to be not reachable by the formulas of Sections 4 and 1.

\section{APPENDICES}

A.1. Proof of Proposition 1.3(ii). Let $\mathrm{Fl}^{q_{1}, \ldots, q_{l}}(E) \rightarrow X$ be the flag bundle parametrizing flags of successive quotients of ranks $q_{1}, \ldots, q_{l}$ of $E$. It is endowed with a sequence of tautological quotients $Q^{i}$ where $\operatorname{rank} Q^{i}=i$. If $\left(q_{1}, \ldots, q_{l}\right)=(l, l-1, \ldots, 1)$ then the projection in the flag bundle is denoted by $\tau_{E}^{l}$. The proof goes as follows. First, one shows the assertion for $J=\emptyset$ and any $I$. We use a commutative diagram

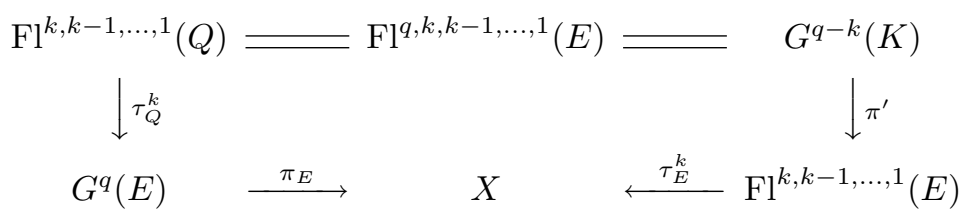

where $K=\operatorname{Ker}\left(E \rightarrow Q^{k}\right)$. Invoking [P3, Corollary 2.7], we have:

$$
\begin{aligned}
\left(\pi_{E}\right)_{*}\left(c _ { \mathrm { top } } \left(R_{E}\right.\right. & \left.\left.\otimes Q_{E}\right) \cdot P_{I}\left(Q_{E}\right) \cap \pi_{E}^{*} \alpha\right)= \\
& =\left(\pi_{E} \tau_{Q}^{k}\right)_{*}\left(a_{1}^{i_{1}} \ldots a_{k}^{i_{k}} \prod_{\substack{1 \leqslant i<j \leqslant q \\
i \leqslant k}}\left(a_{i}+a_{j}\right) \prod_{\substack{1 \leqslant i \leqslant q \\
q<j \leqslant n}}\left(a_{i}+a_{j}\right) \cap\left(\pi_{E} \tau_{Q}^{k}\right)^{*} \alpha\right) \\
& =\left(\tau_{E}^{k}\right)_{*} \pi_{*}^{\prime}\left(c_{\mathrm{top}}\left(R_{K} \otimes Q_{K}\right) \cdot a_{1}^{i_{1}} \ldots a_{k}^{i_{k}} \prod_{\substack{1 \leqslant i<j \leqslant n \\
i \leqslant k}}\left(a_{i}+a_{j}\right) \cap\left(\tau_{E}^{k} \pi^{\prime}\right)^{*} \alpha\right) \\
& =\pi_{*}^{\prime} c_{\mathrm{top}}\left(R_{K} \otimes Q_{K}\right) \cdot P_{I}(E) \cap \alpha .
\end{aligned}
$$

Now, it follows easily from Proposition 1.3(i) that $\pi_{*}^{\prime} c_{\text {top }}\left(R_{K} \otimes Q_{K}\right)$ equals

$$
\operatorname{card}\left\{I \subset(q-k)^{n-q}:|I| \text { even }\right\}-\operatorname{card}\left\{I \subset(q-k)^{n-q}:|I| \operatorname{odd}\right\}
$$

which is the requested multiplicity $d$ in this case.

Passing to the dual Grassmannian, we prove the formula for $I=\emptyset$ and any $J$. If $|I| \geqslant 1$ (and $J$ is arbitrary) we proceed as in [P4, pp. 154-155] with the following changes:

p. 155 l. -6 should read: “ $\ldots=d P_{I^{\prime}, J}\left(R^{\prime}\right) \cap \pi_{2}^{*} \alpha$ ” ,

p. 155 l. -4 should read: “ $\ldots=\pi_{2 *}\left[d \cdot e_{2} P_{I^{\prime}, J}\left(R^{\prime}\right) \cdot \xi^{i_{i}} \cap \pi_{2}^{*} \alpha\right]=d P_{I, J}(E) \cap \alpha$ ".

(Observe that the multiplicity " $d$ " associated with $P_{I^{\prime}, J}\left(R^{\prime}\right)$ is equal to the one associated with $P_{I, J}(E)$.) 
A.2. Proof of Proposition 2.1. We need the following lemma.

Lemma [L2]. Let $A=\left(a_{1}, \ldots, a_{n}\right)$ be a sequence of independent variables. Then the following equality holds

$$
s_{I}\left(a_{1}+1, \ldots, a_{n}+1\right)=\sum_{J \subset I} d_{I J} s_{J}(A),
$$

where $I$ and $J$ denote partitions and

$$
d_{I J}=\operatorname{Det}\left[\left(\begin{array}{c}
i_{p}+n-p \\
j_{q}+n-q
\end{array}\right)\right]_{1 \leqslant p, q \leqslant n} .
$$

(As Lascoux points out, the simplest proof of this identity uses the familiar Binet-Cauchy formula - see, e.g., [L-S1]. These binomial determinants appear as counting certain correlations in $[\mathrm{G} 2]$.

We pass to the proof of Proposition 2.1. Let us decompose formally $E=L_{1} \oplus \ldots \oplus L_{n}$ where rank $L_{i}=1$. Then, according to Schur's Thesis, if $S^{I} E=\bigoplus L_{1}^{t_{1}} \otimes \cdots \otimes L_{n}^{t_{n}}$, then $s_{I}(E)=\sum a_{1}^{t_{1}} \ldots a_{n}^{t_{n}}$, where $a_{i}=c_{1}\left(L_{i}\right)$ and both the sums are taken over the same multiset of sequences $\left(t_{1}, \ldots, t_{n}\right)$. More precisely, $s_{J}(A)=\sum a^{T}$ where the sum runs over all standard tableaux $T$ of shape $J$ filled up with $1, \ldots, n$ and $a^{T}=a_{1}^{t_{1}} \cdot \ldots \cdot a_{n}^{t_{n}}, t_{i}$ being the cardinality of boxes with " $i$ " in $T$ (see, e.g. [M1]). Hence we want to compute the decomposition into Schur polynomials of

$$
\prod_{T}\left(1+\sum_{i} t_{i} a_{i}\right)
$$

where the product is taken over all standard tableaux $T$ of shape $J$ filled up with $1, \ldots, n$. Note that by the assumption we have the following equation of symmetric polynomials in independent variables $x_{1}, \ldots, x_{n}$ :

$$
\prod_{T}\left(\sum_{i} t_{i} x_{i}\right)=\sum_{K} m_{K} s_{K}\left(x_{1}, \ldots, x_{n}\right) .
$$

Using this equation and $\sum t_{i}=|J|$, we get:

$$
\begin{aligned}
\prod_{T}\left(1+\sum_{i} t_{i} a_{i}\right) & =|J|^{-\operatorname{rank}\left(S^{J} E\right)} \prod_{T}\left(|J|+\sum_{i}|J| t_{i} a_{i}\right) \\
& =|J|^{-\operatorname{rank}\left(S^{J} E\right)} \prod_{T}\left(\sum_{i} t_{i}\left(1+|J| a_{i}\right)\right) \\
& =|J|^{-\operatorname{rank}\left(S^{J} E\right)} \sum_{K} m_{K} s_{K}\left(\ldots, 1+|J| a_{i}, \ldots\right) \\
& =|J|^{-\operatorname{rank}\left(S^{J} E\right)} \sum_{K} \sum_{L \subset K}|J|^{|L|} m_{K} d_{K L} s_{L}(E)
\end{aligned}
$$

by the lemma and the homogeneity of Schur polynomials.

A.3. Recursive linear relations for $((\mathbf{J}))$ and $[\mathbf{J}]$. We give here an alternative proof, due to A. Lascoux, of the enumeration of complete quadrics by Schubert $[\mathrm{S}]$ and Giambelli [G2] given in [La-La-T].

Let $A=\left(a_{1}, a_{2}, \ldots\right)$ be a sequence of independent degree 1 indeterminates and let 
$A_{r}=\left(a_{1}, a_{2}, \ldots, a_{r}\right)$. Define two power series in $A_{r}$ :

$$
\begin{aligned}
F_{r} & :=\prod_{1 \leqslant i<j \leqslant r} \frac{1}{1-\left(a_{i}+a_{j}\right)} \\
\text { and } H_{r} & :=\prod_{1 \leqslant i \leqslant j \leqslant r} \frac{1}{1-\left(a_{i}+a_{j}\right)} .
\end{aligned}
$$

Let $\Delta: \mathbb{Z}\left[A_{r+1}\right] \rightarrow \mathbb{Z}\left[A_{r+1}\right]$ denote, in this section, the operator $(-1)^{r} \partial_{1} \circ \ldots \circ \partial_{r}$. Let us make the following change of variables: $b_{1}=-a_{r+1}, b_{2}=-a_{r}, \ldots b_{r+1}=-a_{1}$. Note that with respect to the $b$-variables the above operator becomes $\partial_{\tau} \circ \ldots \circ \partial_{1}$ (i.e. is the operator $\Delta$ studied in Section 4$)$. Denoting $B^{r}=\left(b_{2}, \ldots, b_{r+1}\right)$, we have $\Delta\left(b_{1}^{i} \cdot s_{J}\left(B^{r}\right)\right)=$ $s_{(i-r) J}\left(B_{r+1}\right)$ by Proposition 1.3(i) where $q=1$.

Lemma 1. The following equalities hold:

1) $\Delta\left(F_{r}\right)=F_{r+1}$ if $r$ is even, and 0 - otherwise.

2) $\Delta\left(\left(a_{1}+\ldots+a_{r}\right) \cdot F_{r}\right)=\left(\frac{r+1}{2}-\left(a_{1}+\ldots+a_{r+1}\right)\right) \cdot F_{r+1}$ if $r$ is odd, and $\frac{r}{2} \cdot F_{r+1}$ if $r$ is even.

3) $\Delta\left(H_{r}\right)=\left((r+1)-2\left(a_{1}+\ldots+a_{r+1}\right)\right) \cdot H_{r+1}$.

Proof. 1) We have

$$
\begin{aligned}
F_{r}=F_{r+1}(1 & \left.+b_{1}+b_{2}\right) \ldots\left(1+b_{1}+b_{r+1}\right) \\
& =F_{r+1}\left(\left(1+b_{1}\right)^{r}+\left(1+b_{1}\right)^{r-1} s_{1}\left(B^{r}\right)+\ldots+\left(1+b_{1}\right)^{0} s_{(1)^{r}}\left(B^{r}\right)\right)
\end{aligned}
$$

It follows from the remark preceding the lemma that

$$
\triangle\left(\left(1+b_{1}\right)^{r-j} s_{(1)^{j}}\left(B^{r}\right)\right)=\Delta\left(b_{1}^{r-j} s_{(1)^{j}}\left(B^{r}\right)\right)=(-1)^{j}
$$

for $0 \leqslant j \leqslant r$. Therefore, $\Delta\left(F_{r}\right)=(1-1+1-1+\ldots) F_{r+1}$, where the first factor contains $r+1$ times " \pm 1 ". This yields the assertion.

2) By similar computations we get, for $0<j \leqslant r$, the following equality (for the rest of the proof we use the variables $a_{i}$, set $a:=a_{r+1}$ and adapt a standard $\lambda$-ring notation):

$$
\triangle\left(a(1-a)^{r-j} s_{j}\left(-A_{r}\right)\right)=\Delta\left((r-j) a(-a)^{r-j-1} s_{j}\left(-A_{r}\right)\right)=(-1)^{j+1}(r-j)
$$

Also,

$$
\triangle\left(a(1-a)^{r}\right)=a_{1}+\ldots+a_{r+1}-r .
$$

Taking these equalities into account, we compute

$$
\begin{aligned}
\Delta\left(\left(a_{1}+\right.\right. & \left.\left.\ldots+a_{r}\right) \cdot F_{r}\right)= \\
= & \left(a_{1}+\ldots+a_{r+1}\right) \Delta\left(F_{r}\right)-\Delta\left(a \cdot F_{r}\right) \\
= & \left(a_{1}+\ldots+a_{r+1}\right) \Delta\left(F_{r}\right) \\
& \quad-\Delta\left(a(1-a)^{r}+a(1-a)^{r-1} s_{1}\left(-A_{r}\right)+\ldots+a(1-a)^{0} s_{r}\left(-A_{r}\right)\right) F_{r+1} \\
= & \left(a_{1}+\ldots+a_{r+1}\right) \Delta\left(F_{r}\right) \\
& \quad+\left(r-(r-1)+(r-2)-\ldots \pm 1-\left(a_{1}+\ldots+a_{r+1}\right)\right) F_{r+1} \\
= & \begin{cases}\frac{r}{2} F_{r+1} & \text { if } r \text { is even, } \\
\left(\frac{r+1}{2}-\left(a_{1}+\ldots+a_{r+1}\right)\right) F_{r+1} & \text { if } r \text { is odd. }\end{cases}
\end{aligned}
$$


3) We have

$$
\begin{aligned}
H_{r} & =H_{r+1} \cdot\left(1-a-a_{1}\right) \ldots\left(1-a-a_{r+1}\right) \\
& =H_{r+1} \cdot\left((1-a)^{r+1}+(1-a)^{r} s_{1}\left(-A_{r+1}\right)+\ldots+(1-a)^{0} s_{r+1}\left(-A_{r+1}\right)\right) .
\end{aligned}
$$

By applying $\triangle$ to $(1-a)^{r+1-j} s_{j}\left(-A_{r+1}\right), 0 \leqslant j \leqslant r+1$, one gets a nonzero result only for $j=0,1$ :

$$
\triangle\left((1-a)^{r+1}\right)=-\left(a_{1}+\ldots+a_{r+1}\right)+(r+1)
$$

and

$$
\triangle\left((1-a)^{r} s_{1}\left(-A_{r+1}\right)\right)=-\left(a_{1}+\ldots+a_{r+1}\right) .
$$

Hence $\triangle\left(H_{r}\right)=\left((r+1)-2\left(a_{1}+\ldots+a_{r+1}\right)\right) \cdot H_{r+1}$.

It will be now convenient to use the following notation: for a partition $I=\left(i_{1}, \ldots, i_{r}\right)$, $s_{I}\left(A_{r}\right)=: s\left(\rho_{r-1}+I ; A_{r}\right)$. We define, for the use of this appendix, the numbers $((J))$ and $[J]$ by

$$
\begin{aligned}
& F_{r}=\sum[J] s\left(J ; A_{r}\right) \\
& H_{r}=\sum((J)) s\left(J ; A_{r}\right)
\end{aligned}
$$

(thus $\left.J=\left(j_{1}>j_{2}>\ldots>j_{r-1}>j_{r} \geqslant 0\right)\right)$.

PROPOSITION 2.

(i) The numbers $[J]$ satisfy the following recursive linear relations:

1) $[1,0]=1$

2) $p\left[j_{1}, \ldots, j_{2 p}\right]-\sum_{k}\left[j_{1}, \ldots, j_{k}-1, \ldots, j_{2 p}\right]=$

$$
= \begin{cases}0 & \text { if }\left(j_{2 p-1}, j_{2 p}\right) \neq(1,0), \\ {\left[j_{1}, \ldots, j_{2 p-2}\right]} & \text { if }\left(j_{2 p-1}, j_{2 p}\right)=(1,0) .\end{cases}
$$

We assume the terms with $j_{k+1}=j_{k}-1$ in the above sum to be zero. If $r$ is odd then

$$
\left[j_{1}, \ldots, j_{r}\right]= \begin{cases}{\left[j_{1}, \ldots, j_{r-1}\right]} & \text { if } j_{r}=0 \\ 0 & \text { if } j_{r}>0 .\end{cases}
$$

(ii) The numbers $((J))$ satisfy the following recursive linear relations:

1) $((1,0))=1$

2) $r\left(\left(j_{1}, j_{2} \ldots, j_{r}\right)\right)-2 \sum_{k}\left(\left(j_{1}, \ldots, j_{k}-1, \ldots, j_{r}\right)\right)=$

$$
= \begin{cases}0 & \text { if } j_{r}>0, \\ \left(\left(j_{1}-1, \ldots, j_{r-1}-1\right)\right) & \text { if } j_{r}=0 .\end{cases}
$$

We assume the terms with $j_{k+1}=j_{k}-1$ in the above sum to be zero.

Proof. (i) Since, by the lemma, $\Delta\left(F_{2 p}\right)=F_{2 p+1}$, the coefficient of $s\left(J ; A_{2 p}\right)$ in $F_{2 p}$ is the same as the one of $s\left(J ; A_{2 p+1}\right)$ in $F_{2 p+1}$. In particular, $\left[j_{1}, \ldots, j_{2 p}, j_{2 p+1}\right]=0$ if $j_{2 p+1}>0$, and $\left[j_{1}, \ldots, j_{2 p}, j_{2 p+1}\right]=\left[j_{1}, j_{2}, \ldots, j_{2 p}\right]$ if $j_{2 p+1}=0$. To get 2$)$ we invoke the Pieri formula (we need it for $k=1$ ):

$$
s\left(j_{1}, \ldots, j_{r} ; A_{r}\right) \cdot s_{k}\left(A_{r}\right)=\sum s\left(h_{1}, \ldots, h_{r} ; A_{r}\right)
$$


where the sum is over $h_{1} \geqslant j_{1}>h_{2} \geqslant j_{2}>\ldots>h_{r} \geqslant j_{r}, \sum h_{i}=\sum j_{i}+k$ (see [M1] and [L-S1]). Now writing,

$$
F_{2 p-1}=\sum\left[i_{1}, i_{2}, \ldots, i_{2 p-2}, 0\right] s\left(i_{1}, i_{2}, \ldots, i_{2 p-2}, 0 ; A_{2 p-1}\right),
$$

we express $\Delta\left(F_{2 p-1} \cdot s_{1}\left(A_{2 p-1}\right)\right)$ in two different ways: first, using the Pieri formula and then applying $\triangle$, and secondly, using assertion 2) of the lemma. The comparison of the coefficients of Schur polynomials in the two expressions so obtained gives

$$
\begin{aligned}
p\left[j_{1}, j_{2}, \ldots, j_{2 p}\right]-\sum\left[j_{1}, \ldots, j_{k}-1, \ldots, j_{2 p}\right]= & \text { if }\left(j_{2 p-1}, j_{2 p}\right) \neq(1,0), \\
& = \begin{cases}0 & \text { if }\left(j_{2 p-1}, j_{2 p}\right)=(1,0) .\end{cases}
\end{aligned}
$$

(ii) Using the Pieri formula and the equality $\Delta\left(H_{r-1}\right)=\left(r-2 s_{1}\left(A_{r}\right)\right) H_{r}$ from the lemma, one immediately gets the assertion.

EXAmple 3. 1) $2[5,3,2,1]-[5,3,2,0]-[4,3,2,1]=2 \cdot 4-7-1=0$;

$2[5,4,3,1]-[5,4,3,0]-[5,4,2,1]=2 \cdot 6-5-7=0$

$2[6,3,1,0]-[6,2,1,0]-[5,3,1,0]=2 \cdot 25-10-12=[6,3]=28$.

2) $F_{2}=1+s_{1}+\left(s_{2}+s(2,1)\right)+\left(s_{3}+2 s(3,1)\right)+\left(s_{4}+3 s(4,1)+2 s(3,2)\right)+\ldots \quad$;

$F_{4}=s(3,2,1,0)+3 s(4,2,1,0)+(6 s(5,2,1,0)+4 s(4,3,1,0))+$

$+(10 s(6,2,1,0)+12 s(5,3,1,0)+2 s(4,3,2,0))+$

$+(15 s(7,2,1,0)+25 s(6,3,1,0)+13 s(5,4,1,0)+7 s(5,3,2,0)+s(4,3,2,1))+$

$+(21 s(8,2,1,0)+44 s(7,3,1,0)+32 s(6,4,1,0)+16 s(6,3,2,0)+10 s(5,4,2,0)+$ $+4 s(5,3,2,1))+$

$+(28 s(9,2,1,0)+70 s(8,3,1,0)+87 s(7,4,1,0)+41 s(6,5,1,0)+30 s(7,3,2,0)+$ $+33 s(6,4,2,0)+5 s(5,4,3,0)+10 s(6,3,2,1)+7 s(5,4,2,1))+\ldots$.

3) One has

$((i, j))-((i-1, j))-((i, j-1))=0$ for $j>0$;

$((i, 0))-((i-1,0))-2^{i-1}=0$; hence $((i, 0))=2^{i}-1$.

The number $((i, j)) \quad(i=1,2,3, \ldots, j=0,1,2, \ldots)$ is given in the $i$-th row and $j$-th column of the matrix:

so, for example, $((6,2))=210$.

$\begin{array}{cccc}1 & 0 & 0 & \ldots \\ 3 & 3 & 0 & \ldots \\ 7 & 10 & 10 & \ldots \\ 15 & 25 & 35 & \ldots \\ 31 & 56 & 91 & \ldots \\ 63 & 119 & 210 & \ldots\end{array}$

A.4. A Gysin map proof of the formula of Example 3.5. Let $\tau: \mathcal{F}=$ $\mathrm{Fl}\left(B_{1}, \ldots, B_{k}\right) \rightarrow X$ be the flag bundle parametrizing flags $V_{1} \subset V_{2} \subset \cdots \subset V_{k}$ of vector bundles on $X$ such that $\operatorname{rank} V_{i}=i$ and $V_{i} \subset B_{i}$ for $i=1, \ldots, k$. There is a 
tautological sequence of vector bundles $R_{1} \subset R_{2} \subset \cdots \subset R_{k}$ on $\mathcal{F}$. Let $Z \subset \mathcal{F}$ be a subscheme defined by the vanishing of the homomorphisms:

$$
R_{1} \rightarrow\left(A_{1}\right)_{\mathcal{F}}, \quad R_{2} \rightarrow\left(A_{2}\right)_{\mathcal{F}}, \quad \cdots \quad, R_{k} \rightarrow\left(A_{k}\right)_{\mathcal{F}},
$$

induced by the homomorphism $\varphi: B \rightarrow A$. Of course, $\tau(Z)=\Omega$. Note that $Z$ can be described by a smaller number of equations, i.e., defined by the vanishing of the homomorphisms:

$$
R_{1} \rightarrow\left(A_{1}\right)_{\mathcal{F}}, \quad R_{2} / R_{1} \rightarrow\left(A_{2}\right)_{\mathcal{F}}, \quad \ldots \quad, R_{k} / R_{k-1} \rightarrow\left(A_{k}\right)_{\mathcal{F}}
$$

Let us assume, for a moment, that $X$ is Cohen-Macaulay, $\operatorname{codim}_{\mathcal{F}} Z=n_{1}+\cdots+n_{k}$ and $\tau$ maps $Z$ birationally onto $\Omega$. Then

$$
[Z]=c_{n_{1}}\left(\left(A_{1}\right)_{\mathcal{F}}-R_{1}\right) \cdot c_{n-2}\left(\left(A_{2}\right)_{\mathcal{F}}-R_{2} / R_{1}\right) \cdot \ldots \cdot c_{n_{k}}\left(\left(A_{k}\right)_{\mathcal{F}}-R_{k} / R_{k-1}\right) \cap[\mathcal{F}] .
$$

We have (we omit writing pullback indices and brackets denoting classes of vector bundles in the Grothendieck group, for brevity):

$$
\begin{aligned}
& c_{n_{1}}\left(A_{1}-R_{1}\right) \cdot c_{n_{2}}\left(A_{2}-R_{2} / R_{1}\right) \cdot \ldots \cdot c_{n_{k}}\left(A_{k}-R_{k} / R_{k-1}\right) \\
& =\left|\begin{array}{ccccc}
c_{n_{1}}\left(A_{1}-R_{1}\right) & 0 & 0 & \ldots & 0 \\
* & c_{n_{2}}\left(A_{2}-R_{2}+R_{1}\right) & 0 & \ldots & 0 \\
* & * & c_{n_{3}}\left(A_{3}-R_{3}+R_{2}\right) \ldots & 0 \\
\vdots & \vdots & \vdots & \ddots & \vdots \\
* & * & * & \ldots c_{n_{k}}\left(A_{k}-R_{k}+R_{k-1}\right)
\end{array}\right|
\end{aligned}
$$

where the places under the diagonal can be occupied by arbitrary elements. Consider the $k \times k$ matrix

$$
\left[\begin{array}{ccccc}
c_{n_{1}}\left(A_{1}-R_{1}\right) & c_{n_{1}+1}\left(A_{1}-R_{1}\right) & c_{n_{1}+2}\left(A_{1}-R_{1}\right) & \ldots & c_{n_{1}+k-1}\left(A_{1}-R_{1}\right) \\
c_{n_{2}-1}\left(A_{2}-R_{2}\right) & c_{n_{2}}\left(A_{2}-R_{2}\right) & c_{n_{2}+1}\left(A_{2}-R_{2}\right) & \ldots & c_{n_{2}+k-2}\left(A_{2}-R_{2}\right) \\
c_{n_{3}-2}\left(A_{3}-R_{3}\right) & c_{n_{3}-1}\left(A_{3}-R_{3}\right) & c_{n_{3}}\left(A_{3}-R_{3}\right) & \ldots & c_{n_{3}+k-3}\left(A_{3}-R_{3}\right) \\
\vdots & \vdots & \vdots & \ddots & \vdots \\
c_{n_{k}-(k-1)}\left(A_{k}-R_{k}\right) & c_{n_{k}-(k-2)}\left(A_{k}-R_{k}\right) & \ldots & \ldots & c_{n_{k}}\left(A_{k}-R_{k}\right)
\end{array}\right]
$$

We claim that the determinant of this matrix equals the preceding determinant. To show it we record:

Lemma [J-L]. Let $A_{1}, \ldots, A_{k}$ and $B_{1}, \ldots, B_{k}$ be elements of a $\lambda$-ring equipped with $\lambda$-operations $\lambda^{i}$; assume that $\operatorname{rank} B_{i} \leqslant i-1$. Then the determinant of the matrix

$$
\left[\lambda^{i_{p}-p+q}\left(A_{p}\right)\right]_{1 \leqslant p, q \leqslant k}
$$

remains unchanged if one replaces the argument $A_{p}$ by $\left(A_{p}+B_{q}\right)$ in the $(p, q)$-place.

(More precisely, this is the dual version of a result from [J-L], given originally using Wronski's aleph functions; see the next Appendix and also [L-S1, 7.5]). 
Our claim now follows by adding $R_{i}$ to the argument of the $(i+1)$-th column in the latter matrix.

In order to end the calculation we now invoke the following formula for the Gysin map in $\operatorname{Fl}\left(B_{1}, \ldots, B_{k}\right)$ (see $\left.[\mathrm{K}-\mathrm{L}]\right)$ : for arbitrary integers $p_{1}, \ldots, p_{k}$ and $\alpha \in A_{*}(X)$,

$$
\tau_{*}\left(\operatorname{Det}\left[c_{p_{i}-i+j}\left(A_{i}-R_{i}\right)\right]_{1 \leqslant i, j \leqslant k} \cap \tau^{*} \alpha\right)=\operatorname{Det}\left[c_{p_{i}-m_{i}+j}\left(A_{i}-B_{i}\right)\right]_{1 \leqslant i, j \leqslant k} \cap \alpha .
$$

Applying this formula, we infer

$$
\tau_{*}\left(\operatorname{Det}\left[c_{n_{i}-i+j}\left(A_{i}-R_{i}\right)\right]_{1 \leqslant i, j \leqslant k} \cap[\mathcal{F}]\right)=\operatorname{Det}\left[c_{n_{i}-m_{i}+j}\left(A_{i}-B_{i}\right)\right]_{1 \leqslant i, j \leqslant k} \cap[X],
$$

as desired.

To get the formula in general, let $C=B \oplus A$. Then $B$ is embedded in $C$ via the graph of $\varphi$ and $A$ is a quotient of $C$ via the projection onto the second summand. By a universality property, we have a section $s_{\varphi}: X \rightarrow \bar{X}=\mathrm{Fl}^{n_{1}, \ldots, n_{k}}(C)$ such that the sequence $C \rightarrow A_{1} \rightarrow \ldots \rightarrow A_{k}$ is the pullback of the universal one $C_{\bar{X}} \rightarrow \bar{A}_{1} \rightarrow \ldots \rightarrow \bar{A}_{k}$ on $\bar{X}$. Let $\bar{\Omega} \subset \bar{X}$ be a subscheme defined by the conditions $\operatorname{dim} \operatorname{Ker}\left(\bar{B}_{i} \rightarrow \bar{A}_{i}\right) \geqslant i$, $i=1, \ldots, k$, where $\bar{B}_{i}=\left(B_{i}\right)_{\bar{X}}$. Then $\Omega=s_{\varphi}^{-1} \bar{\Omega}$. Let $\bar{\Omega}^{\circ} \subset \bar{\Omega}$ be an open subset defined by the conditions $\operatorname{dim} \operatorname{Ker}\left(\bar{B}_{i} \rightarrow \bar{A}_{i}\right)=i, i=1, \ldots, k$. Then $\bar{\Omega}^{\circ}$ is nonempty provided $m_{i} \geqslant i, m_{i}-i \leqslant n_{i}$ for $i=1, \ldots, k$ and both the equalities $\bar{B}_{i}=\bar{B}_{i+1}$ and $\bar{A}_{i}=\bar{A}_{i+1}$ do not hold simultaneously for $i=1, \ldots, k-1$. The latter condition can be rewritten as $n_{i}-m_{i} \geqslant n_{i+1}-m_{i+1}+1$ for $i=1, \ldots, k-1$, or equivalently

$$
n_{1}-m_{1}+1 \geqslant n_{2}-m_{2}+2 \geqslant \ldots \geqslant n_{k}-m_{k}+k>0
$$

(we can assume, without loss of generality, the last inequality to be strict because the equation $n_{k}-m_{k}+k=0$ corresponds to a redundant condition). Under these conditions $\bar{\Omega}^{\circ}$ is nonempty and the morphism $\tau: \mathcal{F}=\operatorname{Fl}\left(\bar{B}_{1}, \ldots, \bar{B}_{k}\right) \rightarrow X$ restricted to the subscheme $Z$ defined for the barred data, induces an isomorphism over $\bar{\Omega}^{\circ}$. Moreover, the sections of

$$
\left(\bar{A}_{1}\right)_{\mathcal{F}} \otimes R_{1}^{\vee}, \quad\left(\bar{A}_{2}\right)_{\mathcal{F}} \otimes\left(R_{2} / R_{1}\right)^{\vee}, \quad \ldots \quad, \quad\left(\bar{A}_{k}\right)_{\mathcal{F}} \otimes\left(R_{k} / R_{k-1}\right)^{\vee}
$$

defining $Z$, are independent, i.e. $\operatorname{codim}_{\mathcal{F}} Z=n_{1}+\ldots+n_{k}$. Then the above calculation establishes the formula for $[\bar{\Omega}]$. The general assertion of Example 3.5 for a CohenMacaulay $X$ then follows from the one just obtained because $\bar{\Omega}$ is Cohen-Macaulay and $s_{\varphi}$ is a regular embedding; this implies (see $[\mathrm{F} 1$, Sect. 6,7$]$ ) $[\Omega]=s_{\varphi}^{*}[\bar{\Omega}]$ provided $\operatorname{codim}_{X} \Omega=\operatorname{codim}_{\bar{X}} \bar{\Omega}$.

A.5. An operator proof of the Jacobi-Trudi formula. In the following proof of Proposition 4.4, we use the notation from loc.cit. We use the equality $\partial=\triangle \circ \partial^{\prime}$ and induction on $n$. We have, by the induction assumption:

$$
\begin{aligned}
& \partial^{\prime}\left(a_{1}^{i_{1}+n-1} \ldots a_{n-1}^{i_{n-1}+1} a_{n}^{i_{n}}\right) \\
& =(-1)^{n-1}\left|\begin{array}{cccc}
0 & s_{i_{1}+1}\left(A_{n-1}\right) & \ldots & s_{i_{1}+n-1}\left(A_{n-1}\right) \\
\vdots & \vdots & \ddots & \vdots \\
0 & s_{i_{n-1}-(n-3)}\left(A_{n-1}\right) & \ldots & s_{i_{n-1}+1}\left(A_{n-1}\right) \\
a_{n}^{i_{n}} & a_{n}^{i_{n}+1} & \ldots & a_{n}^{i_{n}+n-1}
\end{array}\right|_{n \times n}
\end{aligned}
$$


Consider now the following $n \times n$ matrix:

$$
\left[\begin{array}{cccc}
s_{i_{1}}\left(A_{n}\right) & s_{i_{1}+1}\left(A_{n}\right) & \ldots & s_{i_{1}+n-1}\left(A_{n}\right) \\
\vdots & \vdots & \ddots & \vdots \\
s_{i_{n-1}-(n-2)}\left(A_{n}\right) & s_{i_{n-1}-(n-3)}\left(A_{n}\right) & \ldots & s_{i_{n-1}+1}\left(A_{n}\right) \\
a_{n}^{i_{n}} & a_{n}^{i_{n}+1} & \ldots & a_{n}^{i_{n}+n-1}
\end{array}\right] .
$$

We claim that the determinant of this matrix equals the previous determinant, i.e. $(-1)^{n-1} \partial^{\prime}\left(a^{I+\rho_{n-1}}\right)$. To show it we recall:

JACOBI-LAscoux Lemma [J-L]. Let $A_{1}, \ldots, A_{k}$ and $B_{1}, \ldots, B_{k}$ be elements of a $\lambda$-ring; assume that rank $B_{i} \leqslant i-1$. Then the determinant of the matrix

$$
\left[s_{i_{p}-p+q}\left(A_{p}\right)\right]_{1 \leqslant p, q \leqslant k}
$$

remains unchanged if one replaces the argument $A_{p}$ by $\left(A_{p}-B_{q}\right)$ in the $(p, q)$-place. Here, $s_{i}(A)=(-1)^{i} \lambda^{i}(-A)$, where $\lambda^{i}$ is the $i$-th $\lambda$-operation in the $\lambda$-ring.

(See also [L-S1, 7.5].)

The above claim is proved by subtracting $a_{n}$ from the argument of the last $n-1$ columns (note that the last row then becomes:

$$
a_{n}^{i_{n}} 0 \ldots 0
$$

so we can fill up the first column in an arbitrary way without changing the determinant).

Finally, since $\Delta\left(a_{n}^{k}\right)=(-1)^{n-1} s_{k-(n-1)}\left(A_{n}\right)$, the final assertion about

$$
\partial\left(a^{I+\rho_{n-1}}\right)=\Delta\left(\partial^{\prime}\left((-1)^{n-1}(\text { last determinant })\right)\right)
$$

follows by the Laplace expansion with respect to the last row and $\Delta(f \cdot g)=f \cdot \Delta(g)$ for $f \in S \mathcal{P}\left(A_{n}\right)$.

A.6. A Schur complex proof of the Giambelli-Thom-Porteous formula. First, we need a certain Schur complex constructed in [Ni].

The notation here is as in Sections 1 and 2 of the present paper. It was proved in $[\mathrm{Ni}]$ that there exists a complex $C_{r}(\varphi)$. such that:

$$
C_{r}(\varphi)_{i}=\bigoplus_{\substack{I \subset(m-r)^{n-r} \\|I|=i}} S^{I^{\sim}}(F) \otimes S^{(m-r)^{n-r} / I}(E)
$$

(ii) for every $\varphi: F \rightarrow E$ and $r \geqslant 0, \operatorname{Supp} C_{r}(\varphi) .=D_{r}(\varphi)\left({ }^{19}\right)$.

$\left(C_{r}(\varphi)\right.$. is the complex denoted by $T_{(m-r)^{n-r}}(\varphi)$ in $[\mathrm{Ni}]$.)

Suppose that $X$ is a smooth scheme. We want to pass from the Chow $\operatorname{ring} A^{*}(X)$ to $\operatorname{Gr} K(X)$, where $\operatorname{Gr} K(X)$ is the graded Grothendieck ring associated with the topological filtration on $K(X)$ (see [F1, 15.1.5]). By loc.cit. we have a functorial morphism of graded rings

$$
\phi: A^{*}(X) \rightarrow \operatorname{Gr} K(X)
$$

$\left({ }^{19}\right)$ Given a complex $C$. of vector bundles on $X$, by $\operatorname{Supp}(C$.) we understand the complement to the set of points $x \in X$ for which $(C .)_{x}$ is an exact complex of vector spaces. 
where, for a subvariety $V \subset X, \phi([V])=\left[\mathcal{O}_{V}\right]$. A fundamental property of this homomorphism is that $\phi_{\mathbb{Q}}$ is an isomorphism (loc.cit., 15.2.16).

Given a (finite) complex $C$. of vector bundles on $X$, we denote by $[C$. $]$ the class $\sum(-1)^{i}\left[C_{i}\right]$ in $\operatorname{Gr} K(X)$.

In particular, we have:

$$
\left[C_{r}(\varphi) \cdot\right]=\sum(-1)^{|I|}\left[S^{I^{\sim}}(F)\right] \cdot\left[S^{(m-r)^{n-r} / I}(E)\right],
$$

the sum over $I \subset(m-r)^{n-r}$.

Claim. Let $D$ be an irreducible (closed) subscheme of a smooth scheme $X$. Let $C$. be a finite complex of vector bundles on $X$ and let $P$ be a homogeneous element in $A^{*}(X)$ of degree $\operatorname{codim}_{X} D$. If $\operatorname{Supp}(C.) \subset D$ and $\phi(P)=[C$.$] , then [D]=q \cdot P$ for some $q \in \mathbb{Q}$.

Indeed, consider the following commutative diagram with the first row exact:

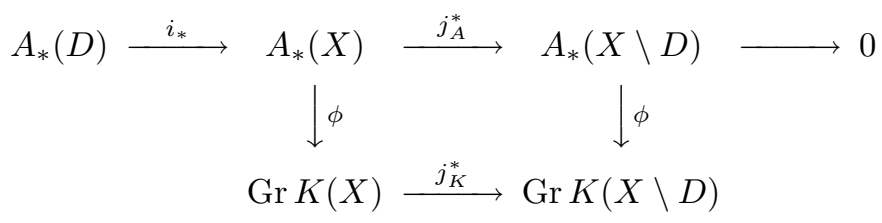

Here, $i: D \rightarrow X$ and $j: X \backslash D \rightarrow X$ denote the inclusions. Since $\operatorname{Supp}(C$. $) \subset D$, we have $j_{K}^{*}([C])=$.0 . Then the equality $\phi(P)=[C$. $]$ implies $\left(j_{A}^{*}\right)_{\mathbb{Q}}(P)=0, \phi_{\mathbb{Q}}$ being an isomorphism. Since $\operatorname{deg} P=\operatorname{codim}_{X} D$ and $D$ is irreducible, $[D]=q \cdot P$ for some $q \in \mathbb{Q}$.

To prove the formula in question, we apply the claim to the triple $D=D_{r}(\varphi)$, $C .=C_{r}\left(\varphi^{\vee}\right)$. and $P=s_{(m-r)^{n-r}}(E-F)$. By passing to a universal case, if necessary, we can assume that $X$ is smooth and the assumptions of the claim are satisfied (see, e.g., [F1, Chap. 14]). We have $\phi(P)=[C$.]. To this end recall that if $L \rightarrow X$ is a line bundle then $\phi\left(c_{1}(L)\right)=\left[\mathbf{1}_{X}\right]-\left[L^{\vee}\right]$. Now, use the splitting principle and write formally $E=\bigoplus_{i=1}^{n} L_{i}$ and $F=\bigoplus_{j=1}^{m} M_{j}$, where $\operatorname{rank} L_{i}=\operatorname{rank} M_{j}=1$. Let $A=\left(a_{1}, \ldots, a_{n}\right)$ with $a_{i}=c_{1}\left(L_{i}\right)$ and $B=\left(b_{1}, \ldots, b_{m}\right)$ with $b_{j}=c_{1}\left(M_{j}\right)$. We must perform the transformation: $a_{i} \mapsto$ $\left[\mathbf{1}_{X}\right]-\left[L_{i}^{\vee}\right]$ and $b_{j} \mapsto\left[\mathbf{1}_{X}\right]-\left[M_{j}^{\vee}\right]$ to the element $s_{(m-r)^{n-r}}(A-B)$. We have by the addition/linearity formula,

$$
s_{(m-r)^{n-r}}(A-B)=\sum(-1)^{|I|} s_{I \sim}(B) \cdot s_{(m-r)^{n-r} / I}(A),
$$

the sum over $I \subset(m-r)^{n-r}$. According to Schur's Thesis, if $S^{I} E=\bigoplus L_{1}^{t_{1}} \otimes \cdots \otimes L_{n}^{t_{n}}$, then $s_{I}(A)=\sum a_{1}^{t_{1}} \ldots a_{n}^{t_{n}}$, where both the sums are taken over the same multiset of sequences $\left(t_{1}, \ldots, t_{n}\right)$. Hence, using (i) for $C_{r}\left(\varphi^{\vee}\right)$., we see that the transformation: $a_{i} \mapsto-\left[L_{i}^{\vee}\right]$ and $b_{j} \mapsto-\left[M_{j}^{\vee}\right]$, sends the element $s_{(m-r)^{n-r}}(A-B)$ to $\left[C_{r}\left(\varphi^{\vee}\right).\right]$. Consequently it suffices to show, that by the change $a_{i} \mapsto a_{i}+1, b_{j} \mapsto b_{j}+1$, the element $s_{(m-r)^{n-r}}(A-B)$ remains unchanged. This is the key point of the argument which follows, e.g., from the fact that the minimal component of the ideal $\mathcal{T}_{r}$ generalizing the resultant (see Theorem 1.5) is generated by $s_{(m-r)^{n-r}}\left(c . / c .^{\prime}\right)$ (for more about that, consult [P4, Sect. 5]). Observe, moreover, that by (ii), $\operatorname{Supp} C_{r}\left(\varphi^{\vee}\right) .=D_{r}(\varphi)$. Hence, by the claim, $\left[D_{r}(\varphi)\right]=q s_{(m-r)^{n-r}}(E-F)$ for some $q \in \mathbb{Q}$. To prove $q=1$, consider a variety $X$ and a morphism $\varphi^{\prime}: F^{\prime} \rightarrow E^{\prime}$ where $\operatorname{rank} E^{\prime}=n-r, \operatorname{rank} F^{\prime}=m-r$ such that $\operatorname{codim}_{X} D_{0}\left(\varphi^{\prime}\right)=(m-r)(n-r)$, $A^{(m-r)(n-r)}(X)$ is a nonzero free abelian group and $s_{(m-r)^{n-r}}(E-F) \neq 0$ (such an example is easily constructable with the help of a Grassmannian). Let $E=E^{\prime} \oplus \mathbf{1}_{X}^{r}$, 


$$
\begin{aligned}
& F=F^{\prime} \oplus \mathbf{1}_{X}^{r} \text { and } \varphi=\varphi^{\prime} \oplus \text { id. Then } \\
& \begin{aligned}
q s_{(m-r)^{n-r}}(E-F) & =\left[D_{r}(\varphi)\right]=\left[D_{0}\left(\varphi^{\prime}\right)\right]=c_{\mathrm{top}}\left(E^{\prime \vee} \otimes F^{\prime}\right)=s_{(m-r)^{n-r}}\left(E^{\prime}-F^{\prime}\right) \\
& =s_{(m-r)^{n-r}}(E-F) .
\end{aligned}
\end{aligned}
$$

This implies $q=1$ and the proof is complete.

\section{A. $\tau$. Corrigenda and addenda to some former author's papers.}

"Every (good) paper must contain an error."

T. Mostowski

We finish this article with a corrigenda and an addenda to the author's former papers [A-L-P], [DC-P], [L-P], [P-P1], [P1-4] and [P-R 2,3]. We apologize for all inconveniences which the misprints, inaccuracies and errors corrected below have caused $\left({ }^{20}\right)$.

[A-L-P]: Misprints - should be:

p. $511_{11}$ — "Let $X$ be a nonsingular" // p. $514^{15}$ — " $\ldots<i_{k} \leqslant n$," //

p. $514_{16}$ - " $\left[i_{1}, \ldots, i_{k}\right]$ " // p. $516^{15}$ — " polynomial in $x_{1}, \ldots, x_{k}, " / /$

p. $517_{11}-" \pi: G / B \rightarrow G / P$ " // p. $517_{10}-" \pi^{-1}\left(Y_{\sigma}\right) " / /$

p. $517_{3}$ - "on $\left(Y_{\sigma} \cap Z\right) \times_{Y_{\sigma}} X_{\sigma \tau}$ "

[DC-P]: Addenda - Here is an elegant argument, pointed out to us by W. Fulton, justifying the footnote on page 688 :

If $\mathcal{L}_{0}$ is a representative of the Poincaré bundle such that $\left.\mathcal{L}_{0}\right|_{\operatorname{Pic}^{2 g-2}(\widetilde{C}) \times\{c\}} \in$ $\operatorname{Pic}^{0}\left(\operatorname{Pic}^{2 g-2}(\widetilde{C})\right)$ and for any $L \in P^{ \pm},\left.\operatorname{Nm} \mathcal{L}_{0}\right|_{\{L\} \times C} \cong \omega_{C}$, then there exists $M \in$ $\operatorname{Pic}^{0}\left(\operatorname{Pic}^{2 g-2}(\widetilde{C})\right)$ such that $\left.\left.\operatorname{Nm} \mathcal{L}_{0}\right|_{P^{ \pm} \times C} \cong p^{*} M \otimes q^{*} \Omega_{C}\right|_{P^{ \pm} \times C}$ where $p: \operatorname{Pic}^{2 g-2} \widetilde{C} \times$ $C \rightarrow \mathrm{Pic}^{2 g-2} \widetilde{C}$ is the projection. Since $\mathrm{Pic}^{2 g-2}(\widetilde{C})$ is an abelian variety, there exists $L \in \operatorname{Pic}^{0}\left(\operatorname{Pic}^{2 g-2}(\widetilde{C})\right)$ such that $M=L^{\otimes 2}$. Then $\mathcal{L}=\mathcal{L}_{0} \otimes\left(p^{\prime}\right)^{*}\left(L^{\vee}\right)$, where $p^{\prime}: \operatorname{Pic}^{2 g-2}(\widetilde{C}) \times \widetilde{C} \rightarrow \operatorname{Pic}^{2 g-2}(\widetilde{C})$ is the projection, does the job.

[L-P]: Misprints - should be:

p. $209_{3}-" 1 \leqslant i_{1}<\ldots<i_{k} \leqslant n, k=1, \ldots " / /$ p. $210^{4}-"\left\{f\left(b_{1}\right), \ldots, f\left(b_{m}\right)\right\} " / /$

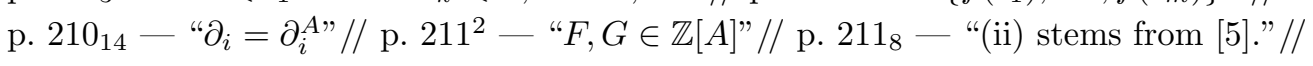

p. $212^{5}$ — " $\left\langle X_{\mu} \omega, X_{\mu \omega}\right\rangle " \quad / /$ p. $212^{13}$ — " $\sum_{i=0}^{\infty} " / /$ p. $212_{15}$ - " $Q_{i}(A) " / /$

p. $215^{8}-" \sigma_{j}^{(i)}: " \quad / /$ p. $215_{6}$ — "Sér. I "

$[\mathrm{P}-\mathrm{P} 1]\left({ }^{21}\right)$ Revision - p. 192: The definition of $((J))$ and $[J]$ was incorrectly reproduced from [P3] and [La-La-T]. For a correct definition, see Section 2 of the present paper.

$\left({ }^{20}\right)$ We do not correct, however, the errors of English because of two reasons. First, this would make this paper too long, and secondly, this will lead, undoubtedly, to ... some new errors.

$\left({ }^{21}\right)$ The following remark applies to [P-P1] and to the earlier papers [P3] and [P4]. Given partitions $I, J$ such that $l(I) \leqslant k$ and $l\left(J^{\sim}\right) \leqslant i$, by $(i)^{k}+I, J$ we denote the partition $\left(i+i_{1}, \ldots, i+i_{k}, j_{1}, j_{2}, \ldots\right)$, i.e., $\left((i)^{k}+I\right), J$ following the literal convention of [P-P1] and 
Addenda - p. 194: Theorem 5 holds under the assumption that $\mathcal{Z}$ is a Whitney stratification; see the paper by A. Parusiński and the author, A formula for the Euler characteristic of the singular hypersurfaces, J. Algebraic Geom. 4 (1995), 337-351.

[P1]: Misprint

p. $250^{9}$ - instead of " $(-1)^{k} c_{k}(A)$ ", should be " $c_{k}(a)$ ".

[P2]: Misprints — should be:

p. $217_{1}-“ \mapsto\left(k\right.$-th elementary " // p. $218^{8}-“ \mapsto(i$-th elementary " //

p. $218^{11}$ — " $\left.\left., \ldots, a_{r+1}-b_{r+1}\right)\right)_{0} " / /$ p. $218_{4}$ — But by $(1.3), s_{I}\left(B^{\prime}-A^{\prime}\right)$ " //

p. $219^{13}$ — "linear ordering" // p. $219^{16}$ — "as the $\mathbb{Z}[c .(a)]$-combination of $s_{J}(B)$ 's" //

p. $219_{6}$ - " $\sum m_{I}\left(A^{\prime}+C\right) s_{I}\left(B^{\prime}-A^{\prime}\right)$ " // p. $221_{7}$ — " diviseur de deux " //

p. $221_{4}$ - "Porteous"

Revisions:

- In this note, by mimicking some (probably not worth recommending) manner, we identify both mathematically and linguistically a polynomial $a(x)$ with the equation " $a(x)=0$ " it determines; consequently, we write exchangeably "polynomial $a(x)$ " or "equation $a(x)$ ".

- In all formulas of the paper, $c_{0}(-)$ is to be understood to be equal to 1 .

- in the proof of Lemma 2.5, $c_{k}\left(a^{\prime}\right)$ (resp. $\left.c_{k}(C)\right)$ means in the analogy to $c_{k}(a)$ and $c_{k}(b)$ the $k$-th elementary symmetric polynomial in $A^{\prime}$ (resp. $C$ ).

[P3]: Here is a list of misprints and revisions (apart of those in [P4, pp. 185-186]):

Misprints - should be:

p. $423^{20}$ — “ $\left(\begin{array}{l}n \\ 2\end{array}\right)$ less " // p. $426^{10}-$ “... $=\left[R_{F}^{\vee} \otimes R_{E}\right]+\left[F^{\vee} \otimes Q_{E}\right] . " / /$

p. $426^{13}-"=\pi_{*}\left[s_{I}\left(Q_{E}\right)\right.$ " // p. $426_{13,15,17}$ - All three instances of " $s_{(m-r)^{n}}$ " should be

"s ${ }_{(m)^{n-r}}$ "// p. 431 $1_{5,10}$ - Lemma 3.6 " // pp. 431, $435_{15}-" s_{(m-r)^{n-r}+I}(E-F) " / /$

p. $435_{10}-" s_{(m-r)^{n-r},(2)}(E-F) " / /$ p. $440_{8}-"[E \otimes Q]-\left[\Lambda^{2} Q\right]=[R \otimes Q]+\left[S_{2} Q\right] " / /$

p. $442_{8}-"[E]=[\operatorname{Im} \varphi]+[C] " / /$ p. $444_{6}-" A(X)$-module structure " //

p. $\left.447^{5}-“ \ldots+\left(\begin{array}{c}j_{p}+j_{q} \\ j_{q}+1\end{array}\right)\right] " / /$ p. $447^{7}-" \sum_{p=1}^{a}(-1)^{p-1} 2^{j_{p}} " / /$ p. $448^{3}-$ "by (30)" //

p. $\left.449_{1}-"\left(E_{i}-F_{i}\right)\right] " / /$ p. $451_{6}-\sum_{r=0}^{i}(-t)^{r} s_{(} \quad " / /$

p. $452^{9}-"\left(a_{r}-a_{s}\right)\left(a_{r}+a_{s}\right)^{-1} " / /$ p. $452_{5}-" \sum_{p=1}^{k}(-1)^{p-1} w("$.

Revisions:

p. $417_{7}$ - before "We ..." insert a footnote We assume here that $A, B$ is a sequence of independent variables, which can be then specialized in a commutative ring.

[P3,4] (as it was explained on the example of the factorization formula in [P3, Lemma 1.1] and $[\mathrm{P} 4$, Proposition 2.2], and then used without further comment). 
pp. $422_{3}-423^{2}$ - should be: "The sequence (8) allows us to treat the following classes of polynomials: symmetric in $A$, symmetric separately in $A^{k}$ and in $A_{n-k}$, and finally symmetric in $A_{n-k}$, as operators respectively on $A(X), A\left(G^{k}(E)\right)$ and finally on $A\left(\mathrm{Fl}^{k}(E)\right)$."

p. 424 - Proposition 2.8 is stated incorrectly for $l(I)=q-1$. (For $l(I)=q$, it is correct as well as the proof given.) The correct formulation for $l(I)=q-1$ is: " $\pi_{*}\left[c_{\mathrm{top}}(R \otimes\right.$ Q) $\left.P_{I}(Q) \cap \pi^{*} \alpha\right]=P_{I}(E) \cap \alpha$ if $\operatorname{rank} R$ is even, and 0 if $\operatorname{rank} R$ is odd ". Since in Section 7 we use precisely this formula when $\operatorname{rank} R$ is even, the correction affects no other results and proofs in the paper. More generally one has for $l(I)=k \leqslant q$

$$
\pi_{*}\left[c_{\mathrm{top}}(R \otimes Q) P_{I}(Q) \cap \pi^{*} \alpha\right]=d P_{I}(E) \cap \alpha,
$$

where $d=0$ if $(q-k)(n-q)$ is odd and $\left(\begin{array}{l}{[(n-k) / 2]} \\ {[(q-k) / 2]}\end{array}\right)$ - otherwise. For details consult Proposition 1.3 (in the present paper).

p. $427^{10}$ - replace the given reference by: "(cf. [F], Theorem $\left.6.2(\mathrm{a})\right)$ "

p. 446 and 447 - By quoting [L-L-T], we were sure that its authors would present a divided-differences proof of Proposition 7.11 independent of the formulas of Proposition 7.12. In the final version of [L-L-T], the authors, however, give the proof (of Proposition 7.11) which makes use of the formulas of Proposition 7.12. For an original, selfcontained, divided-differences proof of Proposition 7.11 due to Lascoux, see Appendix A.3 (in the present paper).

p. 449 - Example (8.3) is revised in Example 3.5 and Appendix A.4 (in the present paper).

[P4]: Here is a list of misprints and revisions:

Misprints - should be $\left({ }^{22}\right)$.

p. $\left.133_{1}-" \leqslant i_{b}+b-1\right\} " \quad / / \quad$ p. $137^{8}-“ \operatorname{sign}(w) w[\quad " / /$

p. $176^{6}$ - the sum is over: “ $\begin{gathered}1 \leqslant i_{1}<\cdots<i_{k} \leqslant n \\ k=0,1, \ldots, n\end{gathered}$ " //

pp. $181_{1}, 182^{3}$ — " P homogen(e)ous symmetric, " // p. 182 $2_{11}$ — “ [G-Z Lemma 8] " // p. $\left.185_{4}-" \cap p_{D}^{*}\left(d_{k}\right)\right)="$.

Revisions:

p. $136^{7}$ - should be: "Move all zeros to the right-hand end, keeping them in order."

p. $137^{12}$ - should be: "was illuminated in [B-G-G] and [D]."

p. 154 - Theorem 3.3 (ii) and its proof are valid if $k=q$. The general case $k \leqslant q$ requires the following correction:

$$
\left(\pi_{E}\right)_{*}\left[c_{\text {top }}\left(R_{E} \otimes Q_{E}\right) P_{J}\left(R_{E}\right) P_{I}\left(Q_{E}\right) \cap \pi_{E}^{*} \alpha\right]=d P_{I, J}(E) \cap \alpha,
$$

where $d=0$ if $(q-k)(n-q-h)$ is odd, and $d=(-1)^{(q-k) r}\left(\begin{array}{c}{[(n-k-h) / 2]} \\ {[(q-k) / 2]}\end{array}\right)$ - otherwise. For more details consult Proposition 1.3 and Appendix A.1 (in the present paper).

p. 179 , Remark 6.11 ; p. 181 , Remark 6.16 - : replace the Chern classes and the Schur polynomials in $R$ by the ones in $R^{\vee}$.

$\left({ }^{22}\right)$ Some readers reported that " $7 . \tau$ " on p. $185_{13}$ is a misprint. Actually, it is not. In the old Mediterranean tradition, the letter $\tau$ means: "to recognize one's error". 
[P-R2]: Revision: In the formula of Proposition 3.5, the factor $\bar{\partial}_{\mu}^{D}\left(f_{\lambda}\right)$ (equal to 1) can be omitted. Then add at the end of the proof of the proposition:

"We get

$$
m_{\mu}=\sum \bar{\partial}_{\mu}^{D}\left(f_{\lambda}\right) \cdot \underline{\partial}_{\mu}^{D}\left(e_{p}\right)
$$

and the assertion follows by the properties of $f_{\lambda}$."

[P-R3]: Misprints — should be:

p. $1036^{20}-" w=\left(\tau, \quad " / / \quad\right.$ p. $1036_{11}-"$ " $+d_{r}, r=1, \ldots, m-n, " \quad / /$

p. 1039 , the bottom picture should have a dot "•" in the last row, i.e., the last two rows of this picture should look like:

p. $1040^{13}$ — " (iii) One has $m_{\mathfrak{b}}=2$."

Revision: p. $1039^{6}$ — better is: "The roof of a deformed (nonextremal) component is its row of highest boxes."

\section{References}

[A-C] E. Akyildiz, J. B. Carrell, An algebraic formula for the Gysin homomorphism from $G / B$ to $G / P$, Illinois J. Math. 31 (1987), 312-320.

[A-L-P] E. Akyildiz, A. Lascoux, P. Pragacz, Cohomology of Schubert subvarieties of $G L_{n} / P$, J. Differential Geom. 35 (1992), 511-519.

[B-G-G] I. N. Bernstein, I. M. Gel'fand, S. I. Gel'fand, Schubert cells and cohomology of the spaces G/P, Russian Math. Surv. 28 (1973), 1-26.

[Bou] N. Bourbaki, Groupes et Algèbres de Lie, Chap. 4, 5 et 6, Herrmann, Paris, 1968.

[B] W. Bruns, Die Divisorenklassengruppe der Restklassenringe von Polynomringen nach Determinantenidealen, Rev. Roumaine Math. Pures Appl. 20 (1975) 11091111.

[Ch] C. Chevalley, Sur les Décompositions Cellulaires des Espaces G/B, Proc. Sympos. Pure Math. 56(1) (1994), 1-23.

[DC-P] C. De Concini, P. Pragacz, On the class of Brill-Noether loci for Prym varieties, Math. Ann. 302 (1995), 687-697.

[D-P-S] J. P. Demailly, T. Peternell, M. Schneider, Compact complex manifolds with numerically effective tangent bundles, J. Algebraic Geom. 3 (1994), 295-345.

[D1] M. Demazure, Invariants symétriques entiers des groupes de Weyl et torsion, Invent. Math. 21 (1973), 287-301.

[D2] M. Demazure, Désingularisation des variétés de Schubert géneralisées, Ann. Sci. École Norm. Sup. (4) 7 (1974), 53-88.

[F1] W. Fulton, Intersection Theory, Springer-Verlag, 1984.

[F2] W. Fulton, Flags, Schubert polynomials, degeneracy loci and determinantal formulas, Duke Math. J. 65 (1992), 381-420.

[F-L] W. Fulton, R. Lazarsfeld, Positive polynomials for ample vector bundles, Ann. of Math. (2) 118 (1983), 35-60.

[G-V] I. Gessel, G. Viennot, Binomial determinants, paths, and hook length formulae, Adv. Math. 58 (1985), 300-321. 
[G1] G. Z. Giambelli, Risoluzione del problema degli spazi secanti, Mem. Accad. Sci. Torino (2) 52 (1903), 171-211.

[G2] G. Z. Giambelli, Il problema della correlazione negli iperspazi, Mem. Reale Istituto Lombardo 19 (1903), 155-194.

[G3] G. Z. Giambelli, Ordine della varietà rappresentata coll'annullare tutti i minori di dato ordine estratti da una data matrice di forme, Acc. Nazion. dei Lincei, Roma, Classe di Science Fis., Mat. e Nat., Rendiconti 12 (1903), 294-297.

[G4] G. Z. Giambelli, Ordine di una varieta piu ampia di quella rappresentata coll'annulare tutti i minori di dato ordine, Memorie Reale Istituto Lombardo 20 (1904), $101-135$.

[G5] G. Z. Giambelli, Risoluzione del problema generale numerativo per gli spazi plurisecanti di una curva algebrica, Mem. Accad. Sci. Torino (2) 59 (1909), 433508.

[H-T1] J. Harris, L. Tu, On symmetric and skew-symmetric determinantal varieties, Topology 23 (1984), 71-84.

[H-T2] J. Harris, L. Tu, Chern numbers of kernel and cokernel bundles, Invent. Math. 75 (1984), 467-475.

[Ha] R. Hartshorne, Ample vector bundles, Inst. Hautes Études Sci. Publ. Math. 29 (1966), 63-94.

[He-T] J. Herzog, Ngô Viêt Trung, Gröbner bases and multiplicity of determinantal and Pfaffian ideals, Adv. Math. 96 (1992), 1-37.

[H-B] H. Hiller, B. Boe, Pieri formula for $S O_{2 n+1} / U_{n}$ and $S p_{n} / U_{n}$, Adv. Math. 62 (1986), 49-67.

[H] F. Hirzebruch, Topological Methods in Algebraic Geometry, Grundlehren der Math. Wissenschaften, Springer-Verlag, 1966; also Collected Papers, vol. I, Springer-Verlag, 1987, 151-334.

[H-H] P. N. Hoffman, J. F. Humphreys, Projective representations of symmetric groups, Oxford University Press, 1992.

[J-L] C. G. Jacobi, A. Lascoux, De quibusdam rationibus universalibus ad determinantia functionalia expedienda, an unpublished manuscript.

[J-L-P] T. Józefiak, A. Lascoux, P. Pragacz, Classes of determinantal varieties associated with symmetric and antisymmetric matrices (in Russian), Izv. Akad. Nauk SSSR 45 (1981), 662-673.

[K-L] G. Kempf, D. Laksov, The determinantal formula of Schubert calculus, Acta Math. 132 (1974), 153-162.

[La] D. Laksov, Remarks on Giovanni Zeno Giambelli's work and life, Rend. Circ. Mat. Palermo (2) Suppl. 36 (1994), 207-218.

[L-L-P-T] D. Laksov, A. Lascoux, P. Pragacz, A. Thorup, a book in preparation.

[La-La-T] D. Laksov, A. Lascoux, A. Thorup, On Giambelli's theorem for complete correlations, Acta Math. 162 (1989), 143-199.

[L1] A. Lascoux, Puissances extérieures, déterminants et cycles de Schubert, Bull. Soc. Math. France 102 (1974), 161-179.

[L2] A. Lascoux, Classes de Chern d'un produit tensoriel, C. R. Acad. Sci. Paris Sér. I Math. 286 (1978), 385-387.

[L3] A. Lascoux, La résultante de deux polynômes, in: Séminaire d'Algèbre Dubreil-Malliavin 1985 (M.-P. Malliavin, ed.), Lecture Notes in Math. 1220, Springer, 1986, 56-72.

[L4] A. Lascoux, Interpolation de Lagrange, in: Second International Symposium (Segovia 1986) "On Orthogonal Polynomials and their Applications", Monograf. Acad. Ci. Exact. Fís.-Quím. Nat. Zaragoza 1 (1988), 95-101. 
[L5] A. Lascoux, Classes de Chern des variétés de drapeaux, C. R. Acad. Sci. Paris 25 (1982), 393-398.

[L6] A. Lascoux, Polynômes de Schubert; une approche historique, Discrete Math. 139 (1995), 303-317.

[La-Le-T1] A. Lascoux, B. Leclerc, J.-Y. Thibon, Une nouvelle expression de functions $P$ de Schur, C. R. Acad. Sci. Paris Sér. I Math. 316 (1993), 221-224.

[La-Le-T2] A. Lascoux, B. Leclerc, J.-Y. Thibon, Fonctions de Hall-Littlewood et polynômes de Kostka-Foulkes aux racines de l'unité, C. R. Acad. Sci. Paris Sér. I Math. 316 (1993), 1-6.

[L-P] A. Lascoux, P. Pragacz, Divided differences and ideals generated by symmetric polynomials, Discrete Math. 126 (1994), 209-215.

[L-S1] A. Lascoux, M.-P. Schützenberger, Formulairé raisonné des functions symétriques, Prepublication L.I.T.P., Université Paris 7, 1985.

[L-S2] A. Lascoux, M.-P. Schützenberger, Symmetry and flag manifolds, in: Invariant Theory (F. Gherardelli, ed.), Lecture Notes in Math. 996, Springer, 1983, 118-144.

[L-S3] A. Lascoux, M.-P. Schützenberger, Polynômes de Schubert, C. R. Acad. Sci. Paris Sér. I Math. 294 (1982), 447-450.

[L-S4] A. Lascoux, M.-P. Schützenberger, Symmetrizing operators on polynomial rings, Functional Anal. Appl. 21 (1987), 77-78.

[L-S5] A. Lascoux, M.-P. Schützenberger, Décompositions dans l'algèbre des differences divisées, Discrete Math. 99 (1992), 165-179.

[L-S6] A. Lascoux, M.-P. Schützenberger, Schubert and Grothendieck polynomials, Notes of the talk given by the first author at Moscow University (November 1987), Preprint L.I.T.P., 1988.

[M1] I. G. Macdonald, Symmetric functions and Hall polynomials, Oxford University Press, 1979.

[M2] I. G. Macdonald, Notes on Schubert polynomials, Publ. LACIM 6, UQUAM, Montréal, 1991.

[Ma] L. Manivel, Un théoreme d'annulation "à la Kawamata-Viehweg", Manuscripta Math. 83 (1994), 387-404.

[Na] V. Navarro Aznar, On the Chern classes and the Euler characteristic for nonsingular complete intersections, Proc. Amer. Math. Soc. 78 (1980), 143-148.

[N] I. Newton, Philosophice Naturalis Principia Mathematica, London, 1687.

[Ni] H. A. Nielsen, Tensor Functors of Complexes, Aarhus Univ. Preprint 15, 1977/78.

[P-P1] A. Parusiński, P. Pragacz, Characteristic numbers of degeneracy loci, in: Enumerative Algebraic Geometry (Copenhagen, 1989), (S. Kleiman, A. Thorup, eds.) Contemp. Math. 123 (1991), 189-197.

[P-P2] A. Parusiński, P. Pragacz, Chern-Schwartz-MacPherson classes and the Euler characteristic of degeneracy loci and special divisors, J. Amer. Math. Soc. 8 (1995), 793-817.

[Po] I. R. Porteous, Simple singularities of maps, in: Proceedings of Liverpool Singularities Symposium I, Lecture Notes in Math. 192, Springer, 1971, 286-307.

[P1] P. Pragacz, Determinantal varieties and symmetric polynomials, Functional Anal. Appl. 21 (1987), 249-250.

[P2] P. Pragacz, A note on elimination theory, Indag. Math. (N.S.) 49 (1987), 215221.

[P3] P. Pragacz, Enumerative geometry of degeneracy loci, Ann. Sci. École Norm. Sup. (4) 21 (1988), 413-454.

[P4] P. Pragacz, Algebro-geometric applications of Schur S-and Q-polynomials, in: Topics in Invariant Theory - Séminaire d'Algèbre Dubreil-Malliavin 1989-1990 (M.-P. Malliavin, ed.), Lecture Notes in Math. 1478, Springer, 1991, 130-191. 
[P5] P. Pragacz, Cycles of isotropic subspaces and formulas for symmetric degeneracy loci, Topics in Algebra, Banach Center Publ. 26(2), 1990, 189-199.

[P-R1] P. Pragacz, J. Ratajski, Polynomials homologically supported on degeneracy loci, Preprint of the University of Bergen No. 61, 1991; to appear in: Ann. Scuola Norm. Sup. Pisa Cl. Sci. (4).

[P-R2] P. Pragacz, J. Ratajski, Pieri type formula for isotropic Grassmannians; the operator approach, Manuscripta Math. 79 (1993), 127-151.

[P-R3] P. Pragacz, J. Ratajski, A Pieri-type formula for $S p(2 m) / P$ and $S O(2 m+1) / P$, C. R. Acad. Sci. Paris Sér. I Math. 317 (1993), 1035-1040.

[P-R4] P. Pragacz, J. Ratajski, A Pieri-type theorem for Lagrangian and odd Orthogonal Grassmannians, Preprint of the Max-Planck Institut für Mathematik 94-15, 1994; to appear in J. Reine Angew. Math.

[P-R5] P. Pragacz, J. Ratajski, Formulas for Lagrangian and orthogonal degeneracy loci; the $\widetilde{Q}$-polynomials approach, Preprint of the Max-Planck Institut für Mathematik, 1994, alg-geom/9602019; to appear in Compositio Math.

[P-T] P. Pragacz, A. Thorup, On a Jacobi-Trudi formula for supersymmetric polynomials, Adv. Math. 95 (1992), 8-17.

[R] J. Ratajski, Thesis, Math. Inst. Polish Acad. Sci., Warsaw, 1995.

[Se] S. Sertöz, A triple intersection theorem for the varieties $S O(n) / P_{d}$, Fund. Math. 142 (1993), 201-220.

[S] H. Schubert, Allgemeine Anzahlfunctionen für Kegelschnitte, Flächen und Raüme zweiten Grades in n Dimensionen, Math. Ann. 45 (1894), 153-206.

[T] R. Thom, Les ensembles singuliers d'une application différentiable et leurs propriétés homologiques, in: Seminaire de Topologie de Strasbourg, December 1957.

[Th] A. Thorup, Parameter spaces for quadrics, in this volume.

$[\mathrm{Tu}]$ L. Tu, Degeneracy loci, Proceedings of the International Conference on Algebraic Geometry (Berlin 1985), Teubner Verlag, Leipzig, 1986, 296-305. 